\title{
Planning Multi-Step Error Detection and Recovery Strategies
}

\author{
Bruce Donald ${ }^{*}$ \\ 87-880 \\ November 1987
}

\author{
Department of Computer Science \\ Cornell University \\ Ithaca, New York 14853-7501
}

\footnotetext{
* This report describes research done at the Artificial Intelligence Laboratory of the Massachusetts Institute of Technology. Support for the Laboratory's Artificial Intelligence research is provided in part by the Office of Naval Research under contract N00014-81-K-0494 and in part by the Advanced Research Projects Agency under Office of Naval Research contracts N00014-85-K-0124 and N00014-82-K-0334. The author was funded in part by a NASA fellowship administered by the Jet Propulsion Laboratory.
} 


\title{
Planning Multi-Step Error Detection and Recovery Strategies
}

\author{
Bruce R. Donald
}

\author{
4158 Upson Hall \\ Computer Science Department \\ Cornell University \\ Ithaca, NY 14853-7501
}

\begin{abstract}
:
Robots must plan and execute tasks in the presence of uncertainty. Incertainty arises from sensing errors, control errors, and uncertainty in the geometry of the environment. By employing a combined strategy of force and position control, a robut programmer can often guarantee reaching the desired final configuration from all the likely initial configurations. Such motion strategies permit robots to carry out tasks in the presence of significant uncertainty. However, compliant motion strategies are very difficult for humans to specify-for this reason we have been working on the automatic synthesis of motion strategies for robots. In previous work [D], we presented a framework for computing one-step motion strategies that are guaranteed to succeed in the presence of all three kinds of uncertainty. The motion strategies comprise sensor-based gross motions, compliant motions, and simple pushing motions.

However, it is not always possible to find plans that are guaranteed to succeed. For example, if tolerancing errors render an assembly infeasible, the plan executor should stop and signal failure. In such cases the insistence on guaranteed success is too restrictive. For this reason we investigate Error Detection and Recovery (EDR) strategies. EDR plans will succeed or fail recognizably: in these more general strategies, there is no possibility that the plan will fail without the executor realizing it. The EDR framework fills a gap when guaranteed plans cannot be found or do not exist: it provides a technology for constructing plans that might work, but fail in a "reasonable" way when they cannot.

We describe techniques for planning nulti-step EDR strategies in the presence of uncertainty. Multi-step strategies are considerably more difficult to generate, and we introduce three approaches for their synthesis: these are the Push-forward Algorithm, Failure Mode Analysis, and the Weak EDR Theory. We have implemented the theory in the form of a planner, called LIMITED, in the domain of planar assemblies.
\end{abstract}


Acknowledgements. This report describes research done at the Artificial Intelligence Laboratory of the Massachusetts Institute of Technology. Support for the Laboratory's Artificial Intelligence research is provided in part by the Office of Naval Research under Office of Naval Research contract N00014-81-K-0494 and in part by the Advanced Research Projects Agency under Office of Naval Research contracts N00014-85-K-0124 and N00014-82-K-0334. The author was funded in part by a NASA fellowship administered by the Jet Propulsion Laboratory. 


\section{Introduction}

Robots must plan and execute tasks in the presence of uncertainty. Incertainty arises from sensing errors, control errors, and uncertainty in the geometric models of the robot and the environment. In this paper we describe a theory of planning multi-step Error Detection and Recovery (EDR) strategies for compliant motion assemblies. We have implemented the theory in the form of a planner, called Limited, in the domain of planar assemblies.

In previous work [D], we addressed two problems. The first is:

(1) The use of active compliance enables robots to accomplish tasks even in the presence of significant sensing and control errors. How can compliant motion strategies be synthesized in the presence of sensing, control, and geometric model error, such that the strategies are guaranteed to succeed so long as the errors lie within the specified bounds? As an example, consider a peg-in-hole assembly with sensing and control uncertainty, with toleranced parts. We wish to synthesize a compliant motion strategy that is guaranteed to suceed so long as the parts lie within the specified tolerances, and the sensing and control errors lie within the specified bounds.

We attacked this problem by introducing additional dimensions to the configuration space; each dimension represented a way in which the parts could parametrically vary. We termed the product space of the motion degrees of freedom and the geometric model variational dimensions "generalized configuration space" and showed how to compute "preimages" [LMT,E] of a geometrical goal in this generalized configuration space. The preimage of a goal is the set of (generalized) configurations from which a particular commanded compliant motion is guaranteed to succeed.

(2) However, it is not always possible to find plans that are guaranteed to suceed. For example, if tolerancing errors render an assembly infeasible, the plan executor should stop and signal failure. In such cases, the insistence on guaranteed success is too restrictive. For this reason we investigated Error Detection and Recovery (EDR) strategies. EDR plans will succeed or fail recognizably: in these more general plans, there is no possibility that the plan will fail without the executor recognizing it. The EDR framework fills a gap when guaranteed plans cannot be found, or do not exist: it provides a technology for constructing plans that might work, but fail in a "reasonable" way when they cannot. In |D|, we gave a constructive, geometric definition of EDR strategies, and showed how thay can be computed for one-step strategies. (A one-step compliant motion strategy is a plan in which a force is commanded in one nominal direction (subject to uncertainty), until certain force- and position-sensing data indicate that the motion should be terminated. In the case of an EDR strategy, the run-time executor must also be able to disanbiguate the result of the motion as a success (achieving the goal) or failure). 


\section{$1.1 \quad$ Examples}

\subsubsection{Application: Planning Gear Meshing}

We must stress that EDR is not limited to problems with model error. There are many applications in which the geometry of the environment is precisely known, but in which guaranteed plans cannot be found, or are very difficult to generate. We now describe such a situation.

An interesting application domain for EDR is gear meshing. It is an example where EDR is applicable even though the shape of the manipulated parts is precisely known. Let us consider a simplified instance of this problem. In fig. 2 there are two planar gear-like objects, $A$ and $B$. The task is to plan a manipulation strategy which will mesh the gears. The state in which the gears are meshed is called the goal.

We will consider two variants of this problem. In the first, we assume that the manipulator has grasped $A$, and that neither $A$ nor $B$ can rotate. However, $A$ can slide along the surfaces of $B$. In the second, $B$ is free to rotate about its center, but this rotation can only be effected by pushing it with $A$. In both cases, the initial orientation of $B$ is unknown. We regard $A$ as the moving object and $B$ as the environment; hence even though the shape of $B$ is precisely known, we choose to view the uncertainty in $B$ 's orientation as a form of model error. In the first case, the system has only two degrees of motion freedom. In the second, there are three degrees of motion freedom, one of which is rotational, since $B$ can be pushed. We distinguish between the rotation and non-rotation variants of the problem in order to highlight the additional techniques our planner employs when rotations are introduced.

In both variations, there is uncertainty in control, so when a motion direction is commanded, the actual trajectory followed is only approximately in that direction. There is also uncertainty in position sensing and force sensing, so that the true position and reaction forces are only known approximately. The magnitude of these uncertainties are represented by error balls.

In general, a commanded motion of $A$ may cause $A$ to move through free space, and contact $B$, possibly causing $B$ to rotate. Our EDR theory is a technique for analyzing these outcomes geometrically to generate strategies that achieve the goal when it is recognizably reachable, and signal failure when it is not.

In an experiment, the EDR theory in the gear domain was applied using the planner, Limited, as follows. Consider the problem of meshing two planar gears, under uncertainty as above. Suppose that gear $B$ can rotate passively but has unknown initial orientation, as above. Suppose that $A$ has been gripped by a robot. The initial position of $A$ is uncertain. The robot can impart either pure forces (translations), or pure torques (rotations) to $A$. The planner can choose the direction of translation or rotation. Can a multi-step strategy of commanded translations and rotations be found to mesh the gears?

Limited was able to generate an EDR strategy for this problem. The characteristics of the experiment are: 

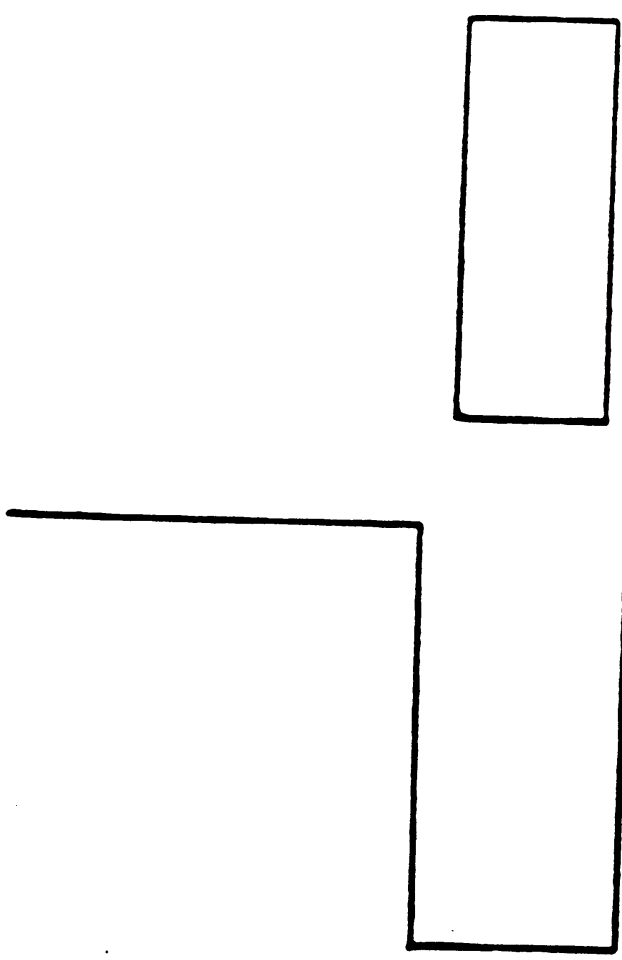

Figure 1: The goal is to insert the peg in the hole. No rotation of the peg is allowed. One can imagine a strategy which attempts to move straight down, but detects contact on the top surfaces of the hole if they occur. If the peg sticks on the top surfaces, the manipulator tries to move to the left or right to achieve the hole. Are these contact conditions "errors"? We maintain that they are not, since they can be planned for and verified. 



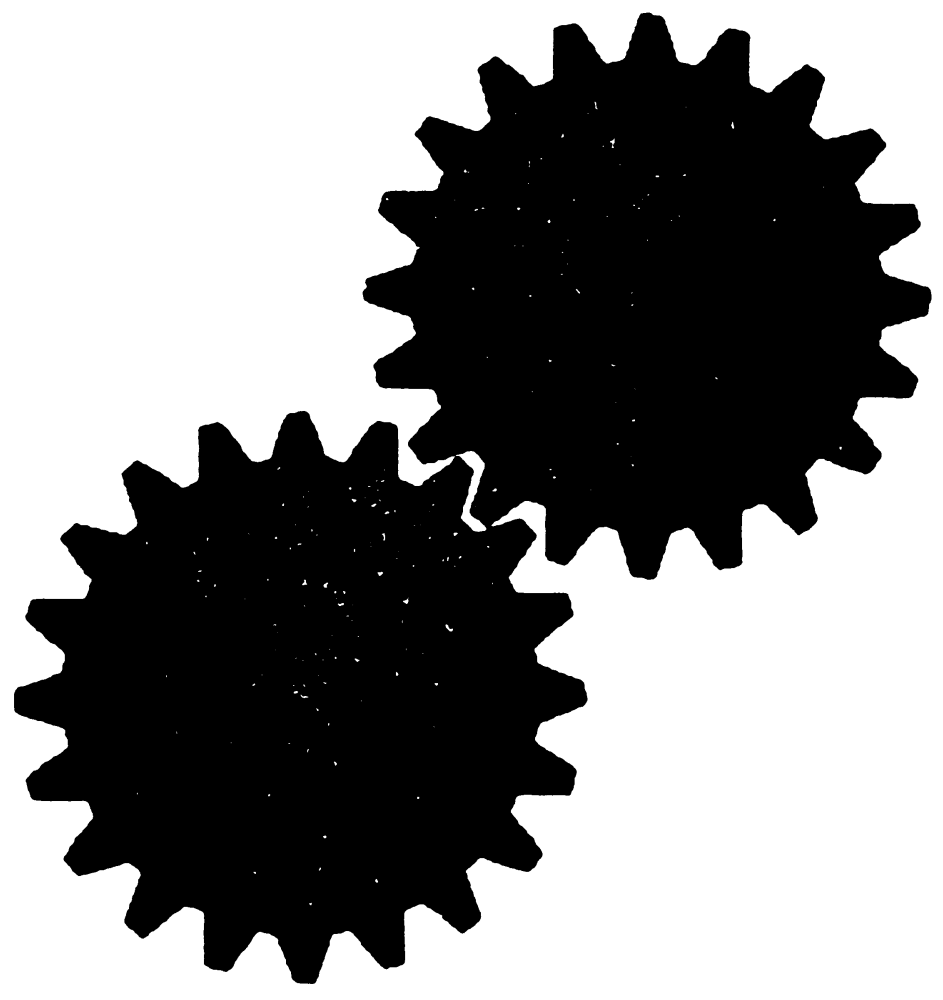

Figure 2: Geometric models of two gear-like planar objects $A$ and $B$. $A$ is grasped and can translate but not rotate. $B$ can rotate about its center if pushed. The orientation of $B$ is unknown. The task is to generate a motion strategy to mesh the gears. 
- There are three degrees of motion freedom (two translational and one rotational) for $A$.

- There is one degree of rotational model error freedom (the orientation of $B$ ).

- It is possible to push $B$ to change its orientation.

- There is sensing and control uncertainty.

- The geometry of the gears is complicated-they have many edges.

- Quasi-static analysis [Mason] is used to model the physics of interaction between the gears.

Thus we have a kind of four-degree of freedom planning problem with uncertainty and pushing. To generate multi-step EDR strategies under pushing, LIMITED employed the EDR theory together with a technique called failure mode analysis.

Now, there may exist a guaranteed strategy to mesh the gears. For example, experimental evidence suggests that for involute teeth gears, almost any meshing strategy will succeed. For other gear shapes perhaps some complicated translation while spinning $A$ will always succeed. I don't know if there is such a guaranteed strategy for this case. It seems difficult for a planner to synthesize such guaranteed strategies, or even to verify them, if they exist at all.

A person might try to solve this problem with the following motion strategy:

- Ram the gears together. See if they mesh.

Or, somewhat more precisely,

- Ram $A$ into $B$. If they mesh, stop. If they jam, signal failure and try again.

Probabilistically, this is a rather good strategy. It is certainly very simple, and probably easier to generate than a guaranteed strategy. If vision can be used to sense whether $A$ and $B$ are meshed, then it is an EDR strategy with just one step.

Suppose, however, that vision is poor, or that the gears are accessible to the robot gripper, but not to the camera. This means that position sensing will be very inaccurate, and hence may be of no use in determining whether the gears are successfully meshed. This will often be the case in practice. In this case, force sensing must be used to disambiguate the success of the motion (meshing) from failure (jamming in an unmeshed state). If the robot has force sensing, then it might use the following two-step EDR strategy:

- Ram the gears together. Spin them to see whether they meshed. 
Or, again more precisely,

- Ram A into B. Next, spin A. If $A$ and B break contact, or if the gears stick (don't rotate), then signal failure. Otherwise, signal success.

This strategy is essentially the one that LIMITED generates. The plan is

Motion 1: Command a pure translation of $A$ into $B .^{1}$

Terminate the motion based on force-sensing when sticking occurs (when there is no motion).

Motion 2: Cornmand a pure rotation of $A$.

If breaking contact or sticking occurs, signal failure. Otherwise, signal success.

In this plan, motion (1) does not terminate distinguishably in success (meshed) or failure (jammed). That is, after motion (1) terminates, the plan executive cannot necessarily recognize whether or not the gears are meshed. LIMITED predicts this, and generates motion (2), which disambiguates the result of motion (1). The generation of the second, disambiguating motion involves the use of failure mode analysis. Breaking contact and sticking are examples of failure modes. The second motion is generated so that from any unmeshed state resulting from motion (1), all possible paths will terminate distinguishably in a failure mode. Failure mode analysis is a robust subtheory of EDR by which LIMITED generates multi-step strategies under pushing.

The theory and implementation behind the generation of motion (1) were discussed extensively in $[D]$. While we will review these techniques briefly, our concern in this paper is how to generate the multi-step strategy above-or more precisely, how to "extend" motion (1) into a 2-step strategy.

\subsubsection{Experiment: Peg-in-Hole with Model Error}

This section describes a plan that was generated by Limited for a peg-in-hole problem with model error. It gives the flavor of how EDR strategies work. Since pushing motions are not involved here, LIMITED does not use failure mode analysis to solve this problem.

Another peg-in-hole problem is depicted in fig. 3. Again, as in fig. 1, there is uncertainty in the width of the hole; that is, the width is known to lie within some given interval. In addition, there are chamfers on the sides of the hole. The depth of the chamfers is also unknown, but we are given bounds on the depth. Finally, the exact

\footnotetext{
${ }^{1}$ LIMITED generates the actual force vector.
} 
orientation of the hole is uncertain. The geometry of the hole is input to the planner as a set of parametrically defined polygons. They are defined by a three parameter family, for width of the hole, depth of the chamfers, and orientation of the hole. An associated bounding interval is also input for each parameter. The geometry of the peg is input as a polygon.

In this problem, the width of the hole may be smaller than the width of the peg. Thus there can exist no strategy that is guaranteed to succeed for all geometric uncertainty values. However, assume that the assembly - the hole geometry-is inaccessible to robust vision or position-measuring devices. In particular, the measurement error will typically determine the model error bounds, which in this example are large for the purpose of illustration. Thus it is not a priori possible to measure the dimensions ahead of time to determine whether or not the assembly is feasible. Instead, the best we can hope for is an EDR strategy: a strategy that takes some action in the world to attempt the assembly, but whose outcome can be recognizably diagnosed as success or failure by the run-time robot executor.

The peg is allowed to translate in the plane. Its motion is modeled using generalized damper dynamics. This permits sliding on surfaces about the hole. Friction is modeled using Coulomb's law. With these dynamics and perfect control, the peg would exhibit straight-line motions in free space, followed by sliding motions in contact, where friction permits. Here, however, there is control uncertainty, which is represented by a cone of velocities. Motions in free space fan out in a kind of "spray." Again, sliding is possible on surfaces, but so is sticking, depending on the effective commanded velocity at a given instant. (In this case, we say sliding is non-deterministic). The size of the control uncertainty cone of velocities is an input to the planner. Whether sticking may occur on an edge may be computed by intersecting the friction cone with the negative control uncertainty cone.

It is possible to sense the position of the peg and the forces acting on it. This information is only approximate. The error bound on the position sensor readings is input to the planner as the radius of a disc.

LIMITED generates plans using a configuration space representation of the constraints |Lozano-Pérez]. In the plane, one imagines shrinking the moving object to a point, and correspondingly "growing" the obstacles. The point must be navigated through freespace, sliding on surfaces, and so forth, into the hole. Fig. 4 shows configuration spaces for different parametric variations of model error. Notice that when the "real" hole is too small for the peg to fit, then there is simply no hole at all in the corresponding configuration space. Each frame in fig. 4 is called a "slice;" a slice represents a cross-section where the model error parameters are constant. To synthesize an EDR strategy, LimiTED must in some sense consider all such slices. In practice Limited works by constructing a finite, although typically large number of slices. We will show how in many cases, only a low polynomial number need be considered. LIMITED begins by considering a small number of slices, and generates a tentative motion strategy. This strategy must pass a test-which 
we call the EDR test-to be recognized as an EDR strategy. One of the chief goals of this thesis is to derive this test, and to make it formal and algorithmic. Next, LIMITED attempts to "generalize" the strategy by considering successively more slices. The strategy is modified so that it passes the EDR test in all slices. The number of slices considered is the resolution of the planning. This approach is called multi-resolution planning.

Let's consider an EDR plan that Limited computed for this problem. Figs. 5-13 show the plan graphically. Qualitatively, the plan may be described as follows:

- (1) First, move left and slightly down. The motion will terminate on the left side of the hole, on the left chamfers, or overshoot the hole entirely. Where the motion terminates depends both on the trajectory evolution within the control uncertainty, and on the actual geometry of the hole. The motion may, however, slide down the left edge of the hole all the way into the goal. However, this sliding is nondeterministic, and the motion may stick anywhere along that edge. Since the first motion may terminate arbitrarily close to the goal region, LIMITED predicts that the run-time executive system cannot necessarily distinguish whether or not the first motion failed to achieve the goal.

- (2) The termination regions from motion (1) are taken as the start regions for a new motion. Next, try to recover by commanding a motion straight down and slightly to the right. This motion may achieve the goal, or may undershoot it, or may overshoot it. The second motion terminates when the peg sticks on a surface. If such a termination surface is outside the goal, it is called a failure region. LIMITED calculates that after the second motion, the failure regions are distinguishable from the goal regions. Hence after the second motion, the run-time executive can recognize whether or not the plan has failed.

Finally, since Limited is a forward-chaining planner, it is possible to take the failure regions from motion (2) and plan a third recovery motion. Thus, roughly speaking, in the EDR framework, recovery actions are planned by forward-chaining from the failure regions of the previous motion. When the failure regions are potentially indistinguishable from the goal (using sensors), then the recovery action must satisfy the formal EDR test when executed from the union of the goal and the previous failure regions. For example, when we view motion strategies as "mappings" between subsets of configuration space, then typical "robust" recovery actions are EDR plans in which the goal is a "fixed point." Motion (2) is an example of such a one-step EDR plan.

Figs. 5-13 show the plan in just four different slices, to give a flavor for the plan. The rest of the slices may be found later in the thesis. Fig. 5 shows the configuration spaces of the four slices. The goal region here is shaded black. Note that in one slice, the goal disappears. The initial uncertainty in the position of the peg is represented by

\footnotetext{
${ }^{2}$ That is, when motion (2) originates in the goal, it also terminates recognizably in the goal.
} 
constraining the reference point (the point to which the peg has been shrunk) to lie in one of the start regions in fig. 6 .

Figs. $7-8$ represents the forward projection of the first motion. This region is the outer envelope of all possible trajectories evolving from the start regions. It is the set of all configurations that are reachable from the start regions, given the commanded velocity and control uncertainty cone.

Fig. 9 shows the termination regions for motion (1). The termination regions outside the goal are not necessarily distinguishable from the goal.

Figs. 10-11 show the forward projection of the second motion.

Fig. 12 shows the termination regions for the second motion.

Fig. 13 shows the size of the position sensing uncertainty ball. The goal and the failure regions in fig. 12 are distinguishable using sensors.

\section{A Review of the EDR Theory}

In this section we review the EDR theory developed in [D]. This review is necessarily somewhat abbreviated. The reader is cautioned that the account below is somewhat intuitive and informal; we attempt to describe the key points of the EDR theory in the plain style, and omit proofs and mention of certain subtle complications. For a detailed development, please refer to [Donald 86, 87, 88].

\subsection{Motivation: Research Issues}

The gross motion planning problem with no uncertainty has received a great deal of attention recently. In this problem, the state of the robot may be represented as a point in a configuration space. Thus moving from a start to a goal point may be viewed as finding an arc in free space connecting the two points. Since the robot is assumed to have perfect control and sensing, any such arc may be reliably executed once it is found. In particular, given a candidate arc, it may be tested. That is, motion along the arc may be simulated to see whether it is collision free. For example, an algebraic curve may be intersected with semi-algebraic sets defining the configuration space obstacles. In the presence of uncertainty, however, we cannot simply simulate a motion strategy to verify it. Instead, we need some technique for simulating all possible orbits, or evolutions of the robot system, under any possible choice of the uncertain parameters. With sensing and control uncertainty, the state of the robot must be viewed as a subset of the configuration space. Motions, then, can be viewed as mappings between these subsets. Of course there are many such subsets! From this perspective, it is clear that a chief contribution of [LMT] has been to identify and give a constructive definition for a privileged class of subsets, called preimages, and show that it is necessary and sufficient to search among this class. This framework appears very promising for planning guaranteed motion strategies 

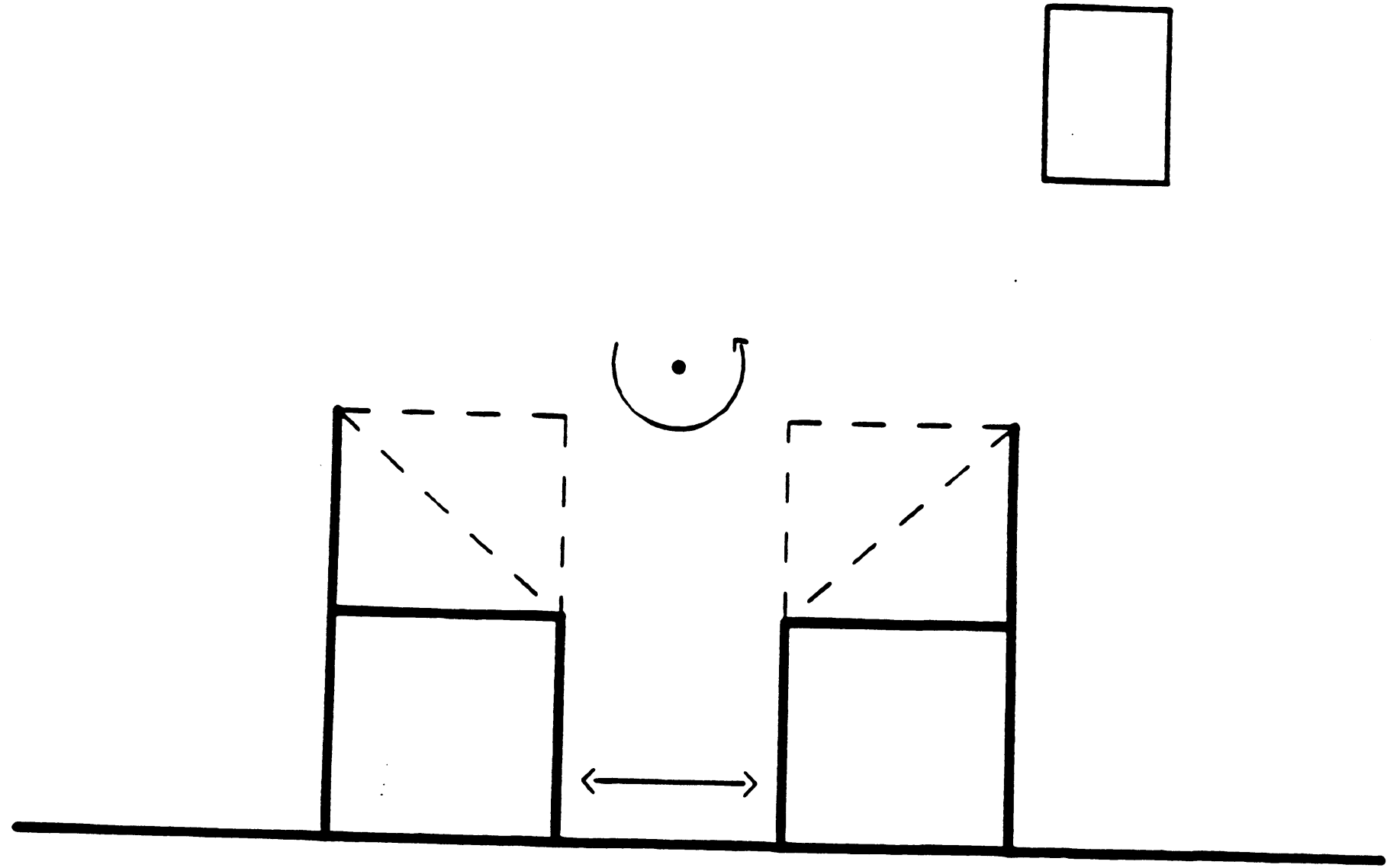

Figure 3: A peg-in-hole environment with model error. The width of the hole $\left(a_{1}\right)$, depth of chamfer $\left(a_{2}\right)$, and orientation of the hole $\left(a_{3}\right)$ are the model parameters. The hole is allowed to close up. 


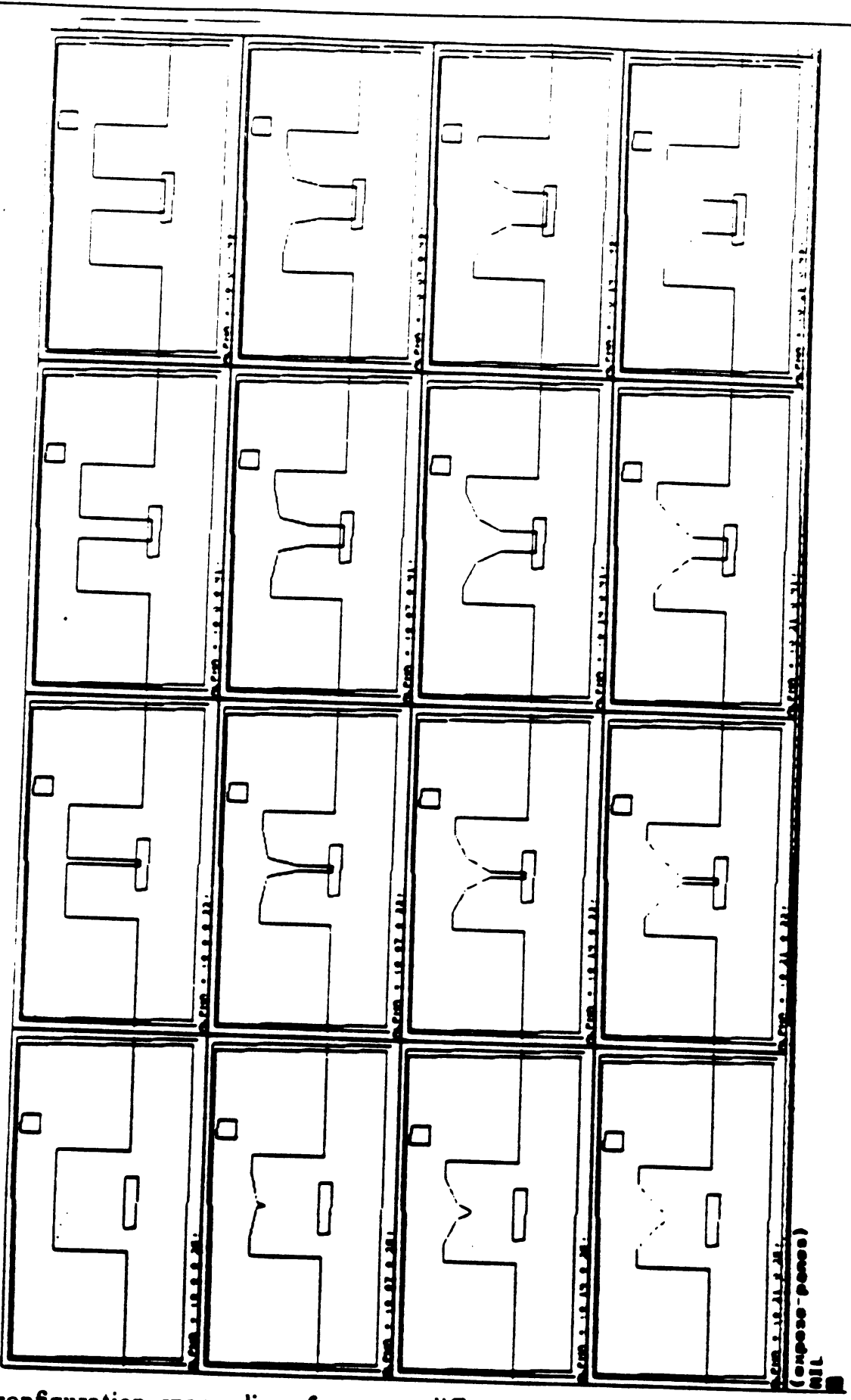

Figure 4: The configuration space slices for many different parametric model error values. These configuration spaces were generated for the peg-in-hole problem with model error depicted in fig. 3. Fig. 4a shows a few slices taken at constant orientation, whereas in fig. $4 \mathrm{~b}$, more slices are shown at various orientations. 


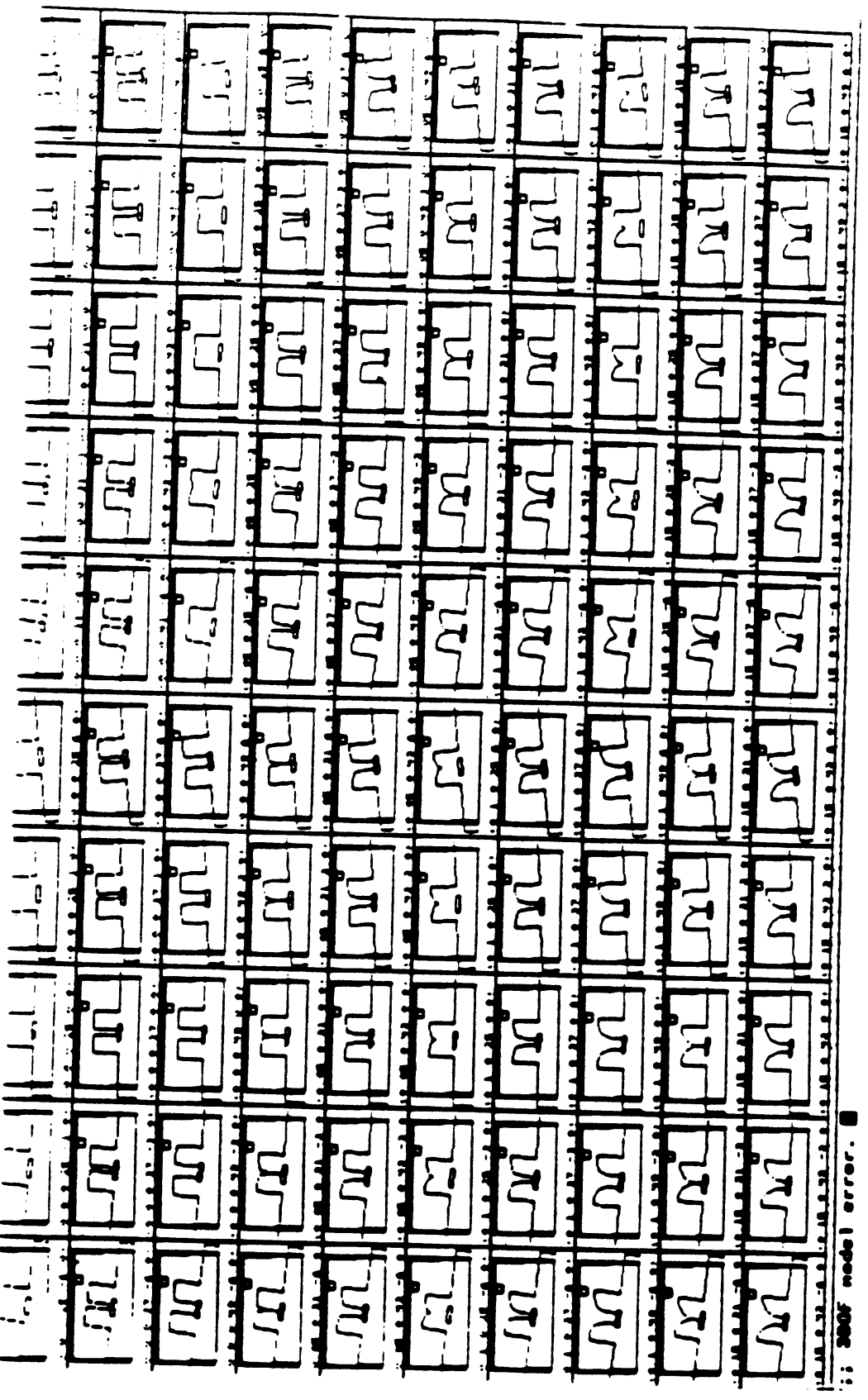

Figure $4 b$. 


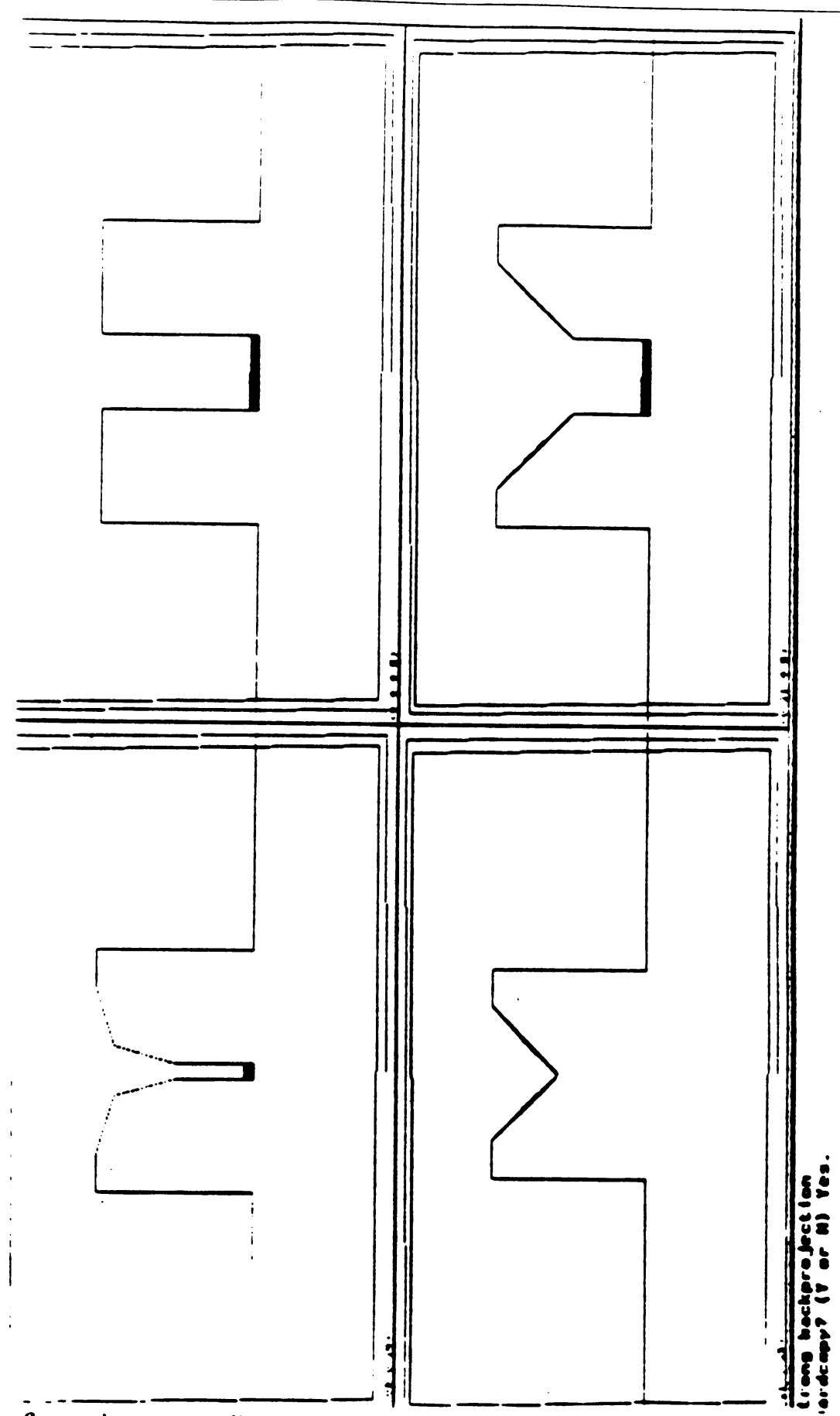

Figure 5: 4 configuration space slices for the peg-in-hole with model error problem. The goal region is shaded black. In one slice, the goal vanishes. 


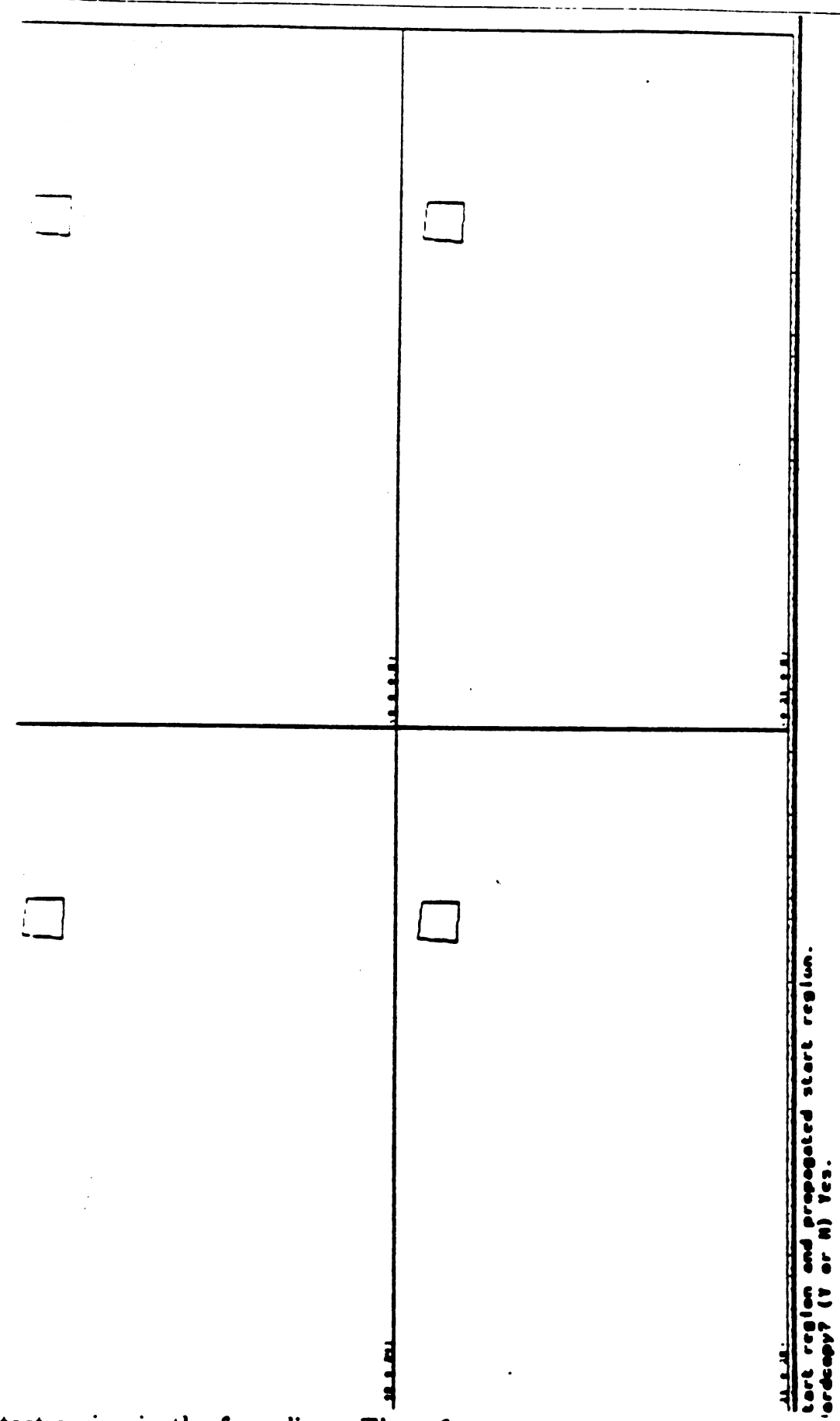

Figure 6: The start region in the four slices. The reference point of the peg is known to start within this region. 


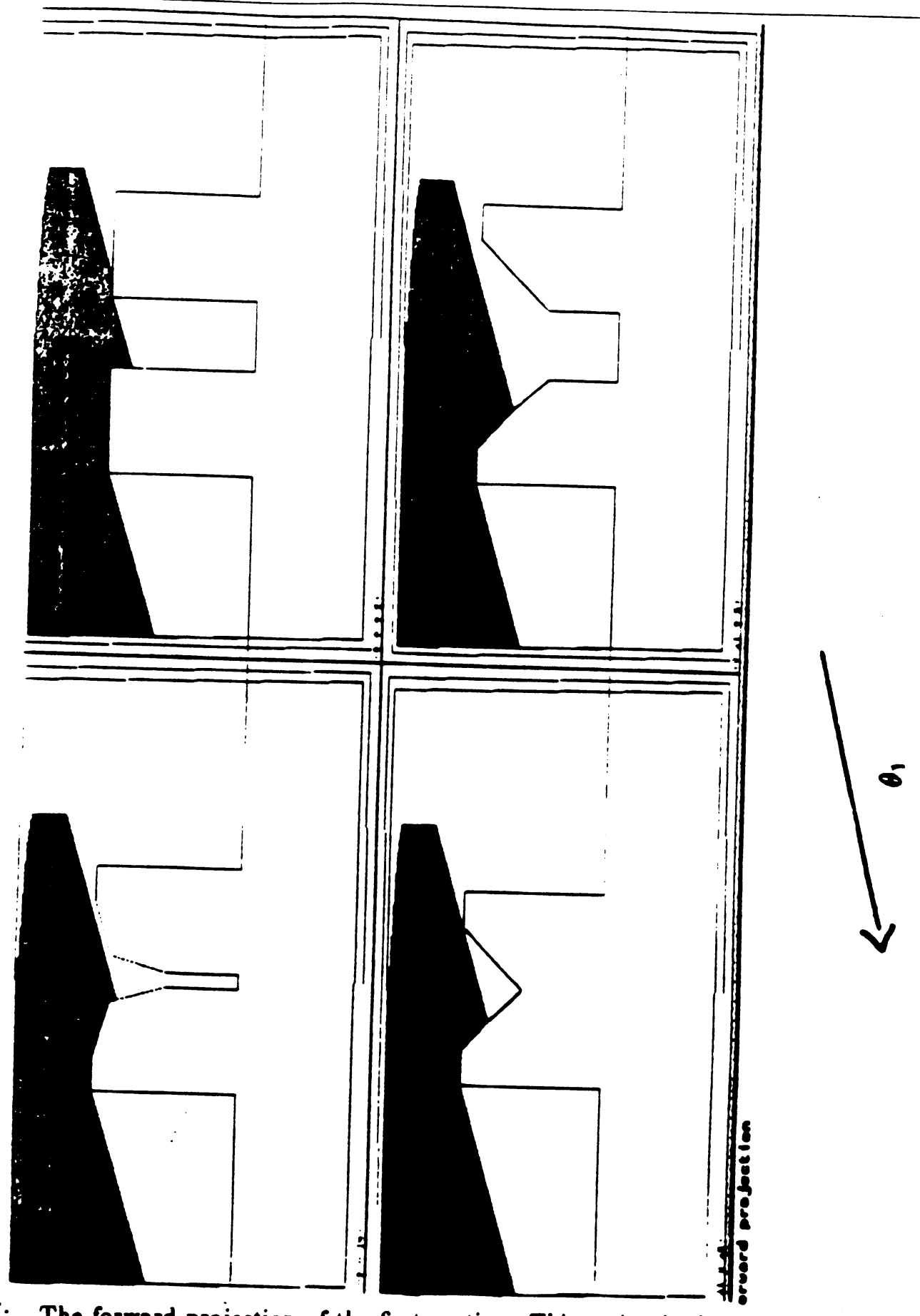

Figure 7: The forward projection of the first motion. This region is the outer envelope of all possible trajectories evolving from the start regions. It is the set of all configurations that are reachable from the start regions, given the commanded velocity and control uncertainty cone. 




Figure 8: The forward projection of the first motion, shown without the obstacles. 


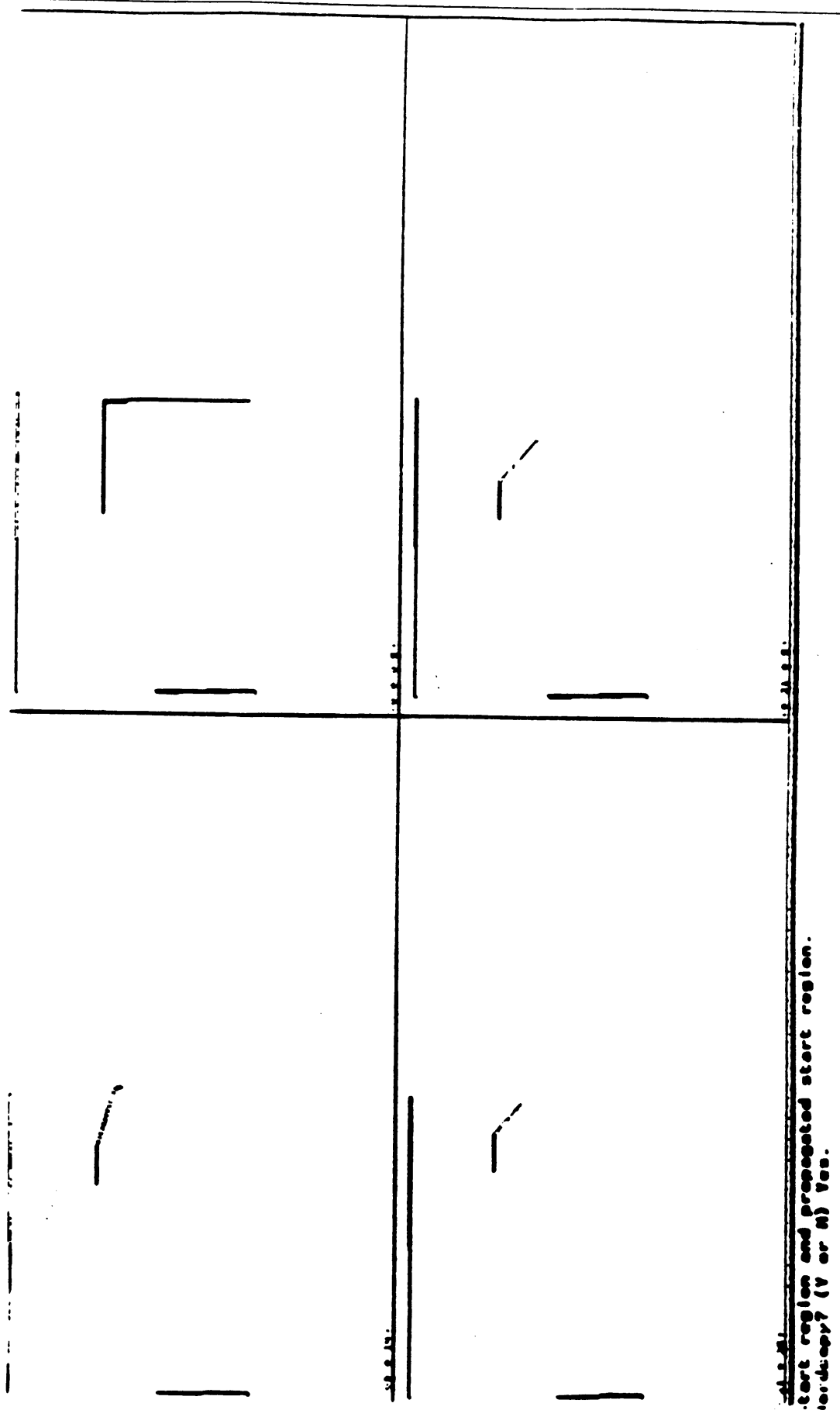

Figure 9: The termination regions from the first motion. These regions are configurations where the motion finishes. 


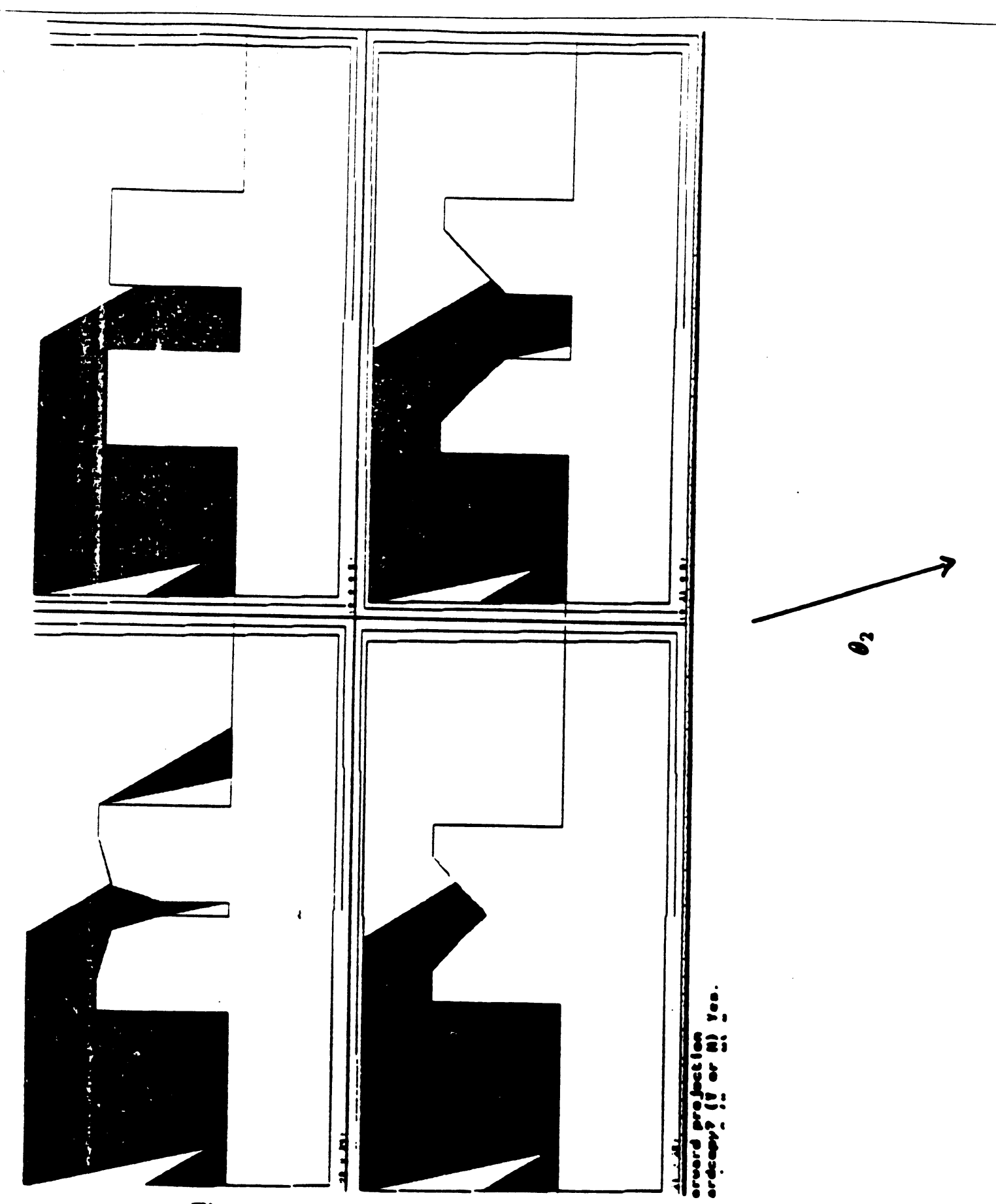

Figure 10: The forward projection of the second motion. 


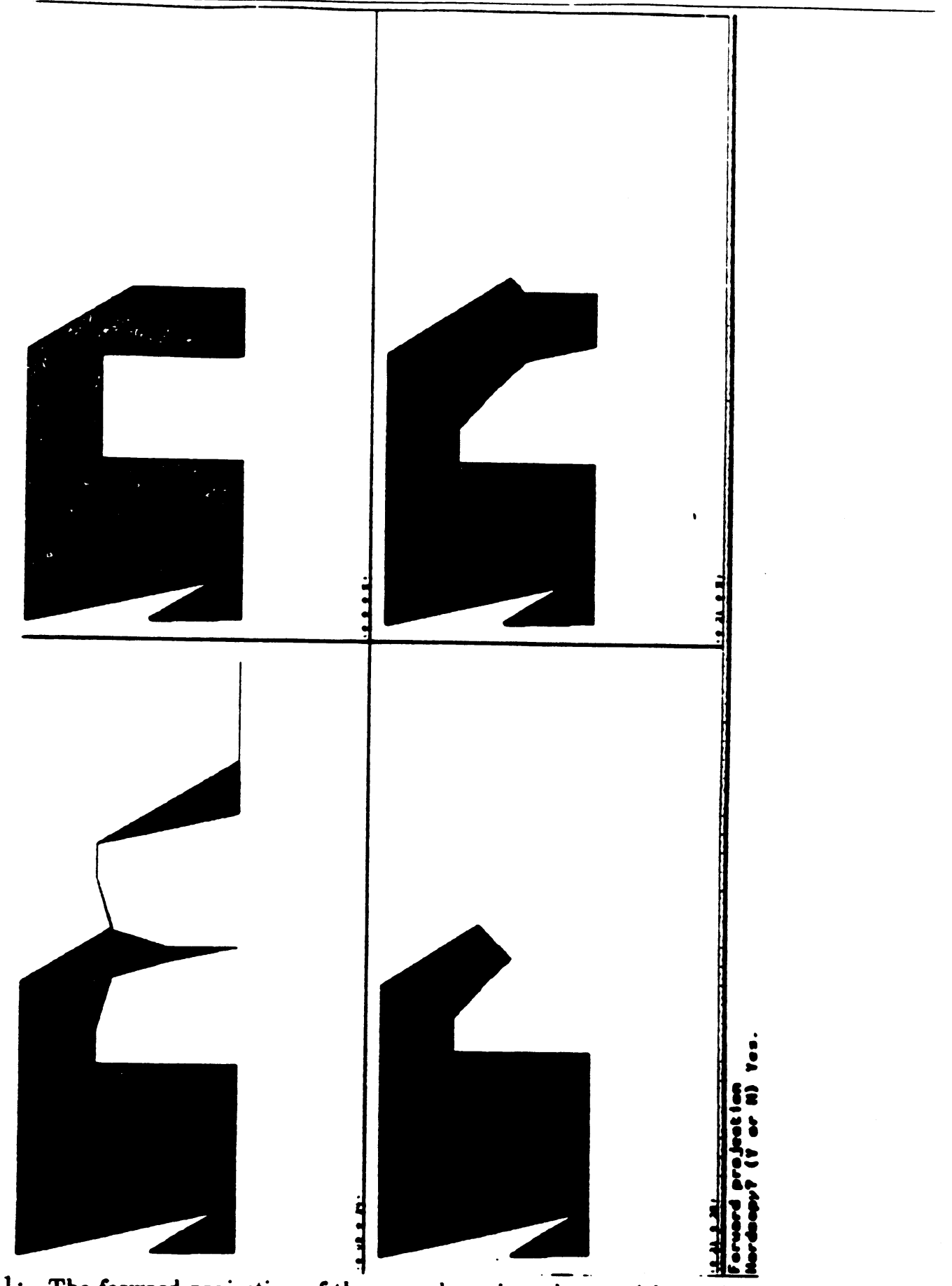

Figure 11: The forward projection of the second motion, shown without the obstacles. 


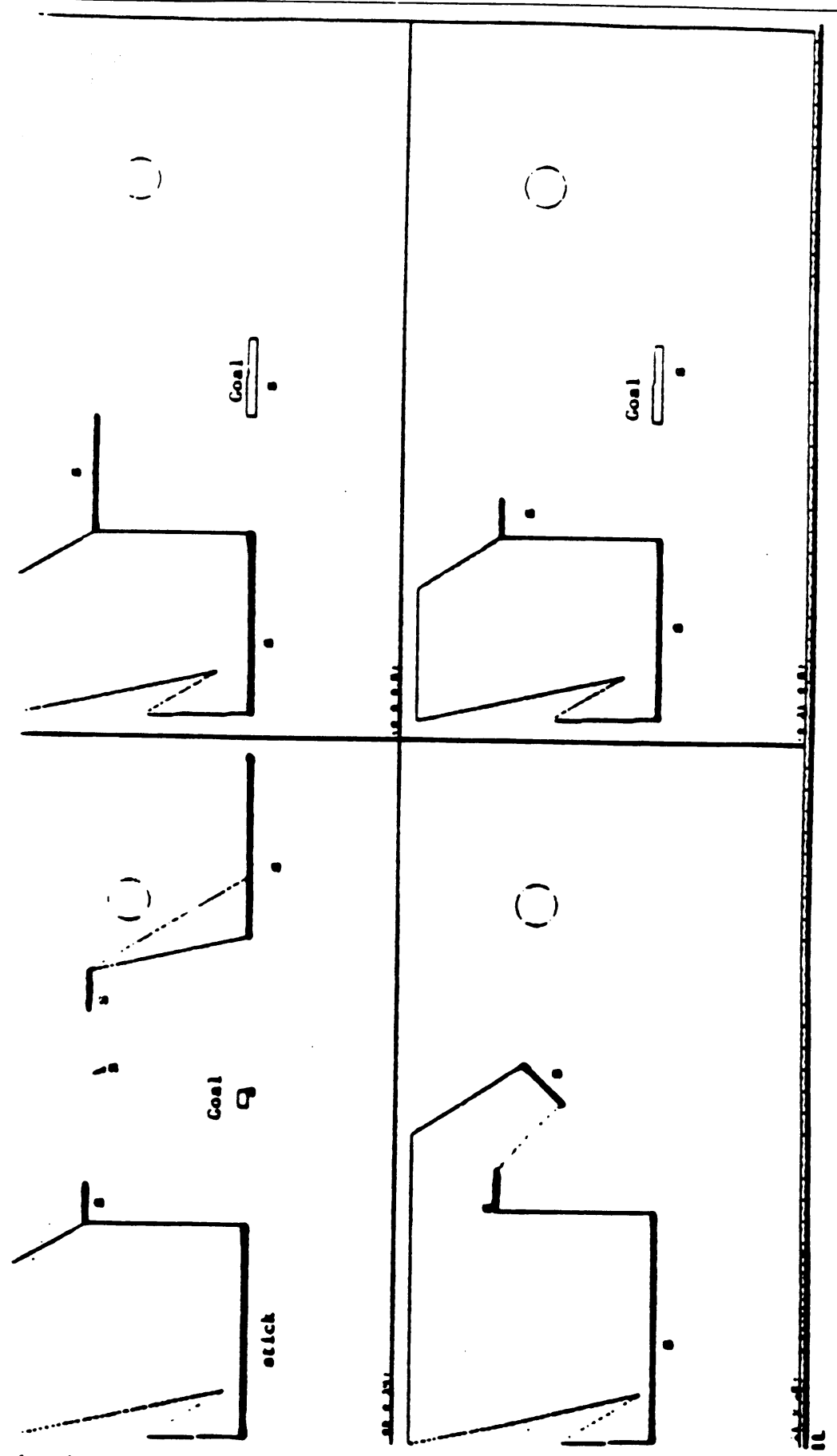

Figure 12: The termination regions for the second motion. These are edges in configuration space where sticking can occur. 


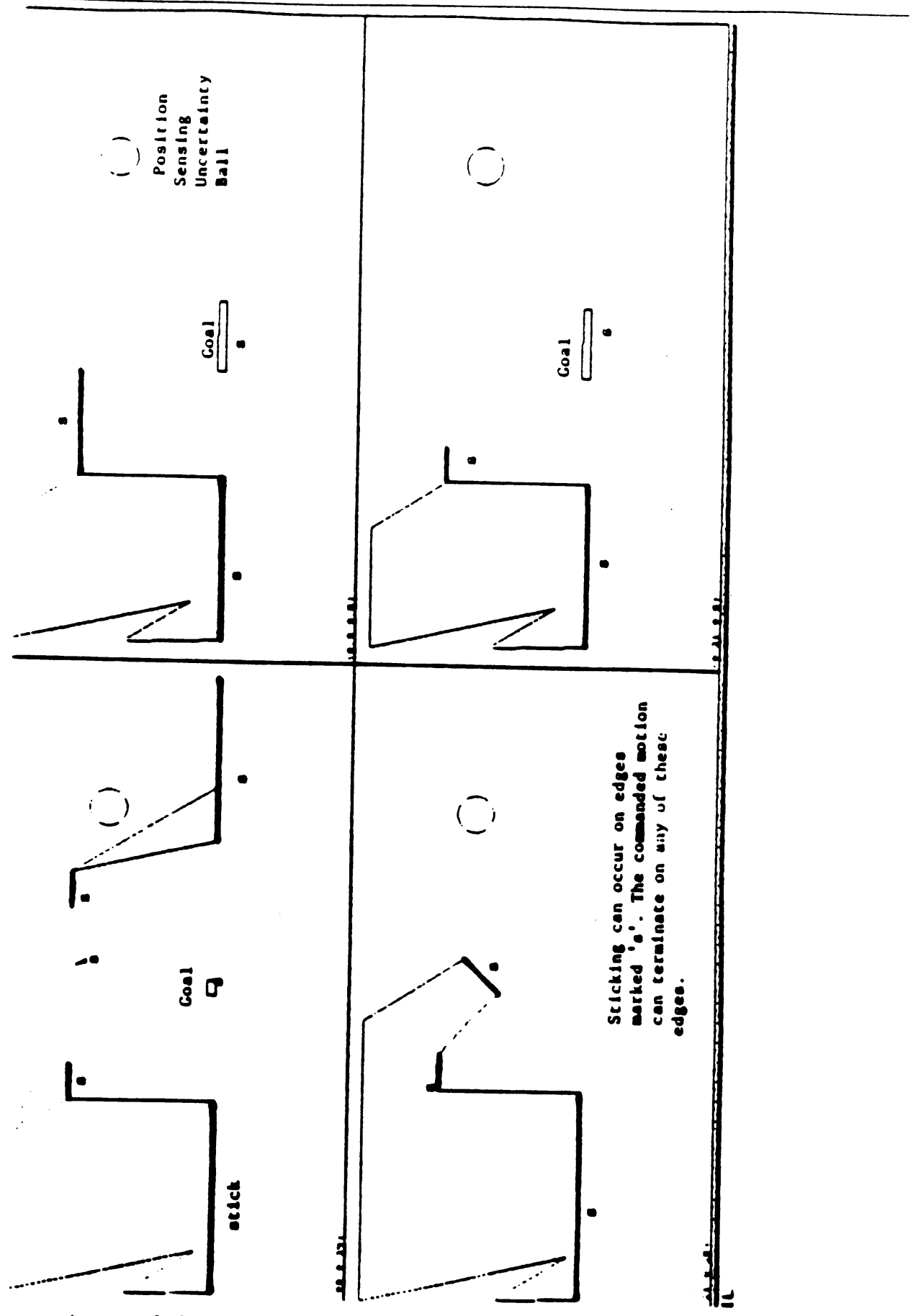

Figure 13: The failure regions and the goal are distinguishable, even given the sensing uncertainty. The disc indicates the magnitude of the position sensing uncertainty. 
under sensing and control uncertainty. The [LMT] framework assumes no model error. In $|D|$, we reduced the problem of planning guaranteed strategies with sensing, control, and geometric model uncertainty to the problem of computing preimages in a (higher dimensional) generalized configuration space. ${ }^{3}$

This result is useful in as much as there was really no prior theory of planning in an environment whose geometry is not precisely known. However, I do not think that it is the main point the EDR theory. This is because there are certain inadequacies with the planning model. The insistence that strategies be guaranteed to succeed is too restrictive in practice. To see this, observe that guaranteed strategies do not always exist. In the peg-in-hole problem with model error (figs. 3-13) there is no guaranteed strategy for achieving the goal, since the hole may be too small for some model error values. For these values the goal in configuration space does not exist. Because tolerances may cause gross topological changes in configuration space, this problem is particularly prevalent in the presence of model error. More generally, there may be model error values for which the goal may still exist, but it may not be reachable. For example, in a variant of the problem in fig. 3, an obstacle could block the channel to the goal. Then the goal is non-empty, but also not reachable. Finally, and most generally, there may be model error values for which the goal is reachable but not recognizably reachable. In this case we still cannot guarantee plans, since a planner cannot know when they have succeeded.

These problems may occur even in the absence of model error. However, without model error a guaranteed plan is often obtainable by back-chaining and adding more steps to the plan. In the presence of model error this technique frequently fails: in the peg-in-hole problem with model error, this technique will not work since no plan of any length can succeed when the hole closes up.

This is why we investigate EDR strategies, and, in particular, attempt to formalize EDR planning. The key theoretical issue is: How can we relax the restriction that plans must be guaranteed to succeed, and still retain a theory of planning that is not completely ad hoc? We attempt to answer this by giving a constructive definition of EDR strategies. In particular, this approach provides a formal test for verifying whether a given strategy is an EDR strategy. The test is formulated as a decision problem about projection sets in a generalized configuration space which also encodes model error. Roughly speaking, the projection sets represent all possible outcomes of a motion (the forward projection), and weakest preconditions for attaining a subgoal (the preimage).

Given the formal test for "recognizing" an EDR strategy, we then tested the definition by building a generate-and-test planner. The generator is trivial; the recognizer is an algorithmic embodiment of the formal test. It lies at the heart of this research. A second key component of the planner is a set of techniques for chaining together motions to synthesize multi-step strategies. The planner is a forward-chaining, multi-resolution planner, called LIMITED. LIMITED operates in a restricted domain. Plans found by LiMITED

\footnotetext{
${ }^{3}$ We use the terms model error and model uncertainty interchangeably.
} 
in experiments are described above.

Finally, let me suggest that a new framework-the EDR framework - for planning with uncertainty may be justified not only by the restrictiveness of the guaranteed-success model, but also by the hardness of the problem. The gross motion planning problem without uncertainty may be viewed, under some very general assumptions, as a decision problem within the theory of real closed fields. This gives a theoretical decision procedure with polynomial running time once the degrees of freedom of the robot system are fixed $[\mathrm{SS} \mid$. However, no such theoretical algorithm is known for the general compliant motion planning problem with uncertainty. Furthermore, the lower bounds for computing guaranteed strategies even in $3 \mathrm{D}$ are dismal: the problem is known to be hard for exponential time $[C R]$. At this point it is unknown whether EDR planning is more efficient than guaranteed planning. However, there is some experimental evidence leading one to conjecture that certain problens requiring very complicated, exponential-sized guaranteed plans may admit very short EDR plans.

However, the motivation for this work is not complexity-theoretic. Instead, the chief thrust is to show how to compute motion strategies under model error (and sensing and control uncertainty), using a formal and constructive definition of EDR strategies. The first goal was a precise geometric characterization of EDR planning--when one thinks about it, it is in fact somewhat surprising that such a thing should exist at all! But in fact it does, as we shall see. 'The second goal was to test this characterization by building a planner. Thus it was necessary to devise implementable algorithms to construct the geometric projection sets and decide questions about them. Therefore, this theory and LiMITED contain a mixture of precise combinatorial algorithrns and of approximation algorithms. In [Donald, 87] we indicate which algorithms are exact and give combinatorial bounds. We also identify the approximation algorithms, and indicate the goodness of the approximation and whether it is conservative. Much work, or course, remains in developing better algorithms for EDR planning, and in testing out the plans using real robots.

\subsection{Representing Model Error}

We will review the EDR theory by examining some very simple planning problems with model error. Of course, this does not mean that EDR is limited to situations with model error.

\subsubsection{A Simple Example: The Variable-Width Peg-In-Hole}

Example (3). Consider fig. 14. There is position sensing uncertainty, so that the start position of the robot is only known to lie within some ball in the plane. The goal is to bring the robot in contact with the right vertical surface of $A$.

We will simplify the problem so that the computational task is in configuration space. 
This transformation reduces the planning task for a complicated moving object to navigating a point in configuration space. Consider fig. 15. The configuration point starts out in the region $R$, which is the position sensing uncertainty ball $B_{\text {ep }}$ about some initial sensed position. To model sliding behavior, we will assume Coulomb friction and generalized damper dynamics, which allows an identification of forces and velocities. Thus the commanded velocity $v_{0}$ is related to the effective velocity $v$ by $f=B\left(v-v_{0}\right)$ where $f$ is the effective force on the robot and $B$ is a scalar. Given a nominal commanded velocity $v_{0}^{*}$, the control uncertainty is represented by a cone of velocities ( $B_{e c}$ in the figure). The actual commanded velocity $v_{0}$ must lie within this cone.

The goal in fig. 15 is to move to the region $G$. Now, with Coulomb friction, sticking occurs on a surface when the (actual) commanded velocity points into the friction cone. We assume the friction cones are such that sliding occurs (for all possible commanded velocities in $B_{e c}$ ) on all surfaces save $G$, where all velocities stick. We will assume that the planner can monitor position and velocity sensors to determine whether a motion has reached the goal. Velocity sensing is also subject to uncertainty: for an actual velocity $v$, the sensed velocity lies in some cone $B_{\text {ev }}$ of velocities about $v$.

Now we introduce simple model error. The shape of $A$ and $B$ are known precisely, and the position of $A$ is fixed. However, the position of $B$, relative to $A$ is not known. $B$ 's position is characterized by the distance $\alpha$. If $\alpha>0$ the goal is reachable. But if $\alpha=0$, then the goal vanishes. No plan can be guaranteed to succeed if $\alpha=0$ is possible. Suppose we allow $\alpha$ to be negative. In this case the blocks meet and fuse. Eventually, for sufficiently negative $\alpha, B$ will emerge on the other side of $A$. In this case, the goal "reappears," and may be reachable again. ${ }^{5}$ Let us assume that $\alpha$ is bounded, and lies in the interval $\left|-d_{0}, d_{0}\right|$.

Our task is to find a plan that can attain $G$ in the cases where it is recognizably reachable. Such a plan is called a guaranteed strategy in the presence of model error. But the plan cannot be guaranteed for the $\alpha$ where the goal vanishes. In these cases we want the plan to signal failure. Loosely speaking, a motion strategy which achieves the goal when it is recognizably reachable and signals failure when it is not is called an Error Detection and Recovery (EDR) strategy. Such strategies are more general than guaranteed strategies, in that they allow plans to fail.

To represent model error, we will choose a parameterization of the possible variation in the environment. The degrees of freedom of this parameterization are considered as additional degrees of freedom in the system. For example, in fig. 15, we have the $x$ and $y$ degrees of freedom of the configuration space. In addition, we have the model error parameter $\alpha$. A coordinate in this space has the form $(x, y, \alpha)$. The space itself is the cartesian product $\Re^{2} \times\left[-d_{0}, d_{0}\right]$. Each $\alpha$-slice of the space for a particular $\alpha$ is a configuration space with the obstacles $A$ and $B$ instantiated at distance $\alpha$ apart. Fig. 15

\footnotetext{
${ }^{4}$ See [Mason 81] for a detailed description of generalized damper dynamics.

${ }^{5}$ This model is adopted for the purposes of exposition, not for physical plausibility. It is not hard to model the case where the blocks meet but do not fuse.
} 
is such a slice.

More generally, suppose we have a configuration space $C$ for the degrees of freedom of the moving object. Let $J$ be an arbitrary index set which parameterizes the model error. (Above, $J$ was $\left[-d_{0}, d_{0} \mid\right)$. Then the generalized configuration space with model error is $(, \times J$. One way to think of this construction is to imagine a collection of possible "universes", $\left\{C_{\alpha}\right\}$ for $\alpha$ in $J$. Each $C_{\alpha}^{\prime}$ is a configuration space, containing configuration space obstacles. The ambient space for each $C_{\alpha}$ is some canonical $C$. $C \times J$ is simply the natural product representing the ambient space of their disjoint union. There is no constraint that $J$ be finite or even countable. In fig. $3, C$ is again the cartesian plane, and $J$ is a three-dimensional product space. One of the $J$ dimensions is circular, to parameterize the angular variation represented by $\alpha_{3}$.

In fig. 16 we show the generalized configuration space for example (1). Note that the goal in generalized configuration space becomes a 2 -dimensional surface, and the obstacles are 3 -dimensional polyhedra. Note that the goal surface vanishes where $A$ and $B$ meet.

Given a configuration space corresponding to a physical situation, it is well known how to represent motions, forces, velocities, and so forth in it (eg., see [Arnold]). The representations for classical mechanics exploit the geometry of differentiable manifolds. We must develop a similar representation to plan motions, forces, and velocities in generalized configuration space. Henceforth, we will denote the generalized configuration space $C \times J$ by $\mathcal{G}$. We develop the following "axioms" for "physics" in $\mathcal{G}$.

1. At execution time, the robot finds itself in a particular slice of $\mathcal{G}$, (although it may not know which). Thus we say there is only one "real" universe, $\alpha_{0}$ in $^{6} J$. This $\alpha_{0}$ is fixed. However, $\alpha_{0}$ is not known a priori. Thus all motions are confined to a particular (unknown) $\alpha_{0}$-slice, such as fig. 15. This is because motions cannot move between universes. In fig. 16, any legal motion in $\mathcal{G}$ is every where orthogonal to the $J$-axis and parallel to the $x-y$ plane.

2 . Suppose in any $\alpha$-slice the position sensing uncertainty ball about a given sensed position is some set $B_{\text {ep. }}$. The set $R$ in fig. 15 is such a ball. We cannot sense across $J$ : position sensing uncertainty is infinite in the $J$ dimensions. ${ }^{7}$ Thus the position sensing uncertainty in $\mathcal{G}$ is the cylinder $B_{\text {ep }} \times J$. In figs. 15,16 , this simply says that $x$ and $y$ are known to some precision, while $\alpha$ is unknown. The initial position in fig. 15 is given by $R \times\left[-d_{0}, d_{0} \mid\right.$. This cylinder is a 3 -dimensional solid, orthogonal to the $x-y$ plane and parallel to the $J$-axis in fig. 16 .

3. Suppose in the configuration space $C$, the velocity control uncertainty about a given nominal commanded velocity is a cone of velocities $B_{e c}$. Such a cone is shown in fig.

\footnotetext{
${ }^{6} \alpha_{0}$ is a point in the multi-dimensional space $J$.

${ }^{7}$ One generalization of the framework would permit and plan for sensing in $J$. In this case one would employ a bounded sensing uncertainty ball in the $J$ dimensions.
} 



Figure 14: The goal is to bring the robot into contact with the right vertical surface of $A$. (For example, the "robot" could be a gripper finger). There is position sensing uncertainty, so in the start position the robot is only known to lie within some uncertainty ball. There is also control uncertainty in the commanded velocity to the robot. It is represented as a cone, as shown. 


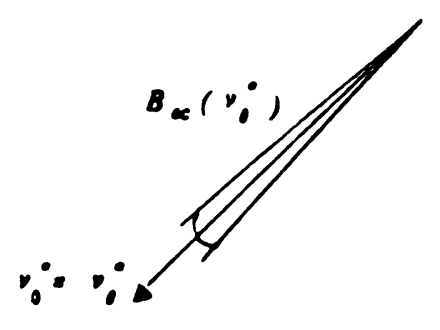

(B)

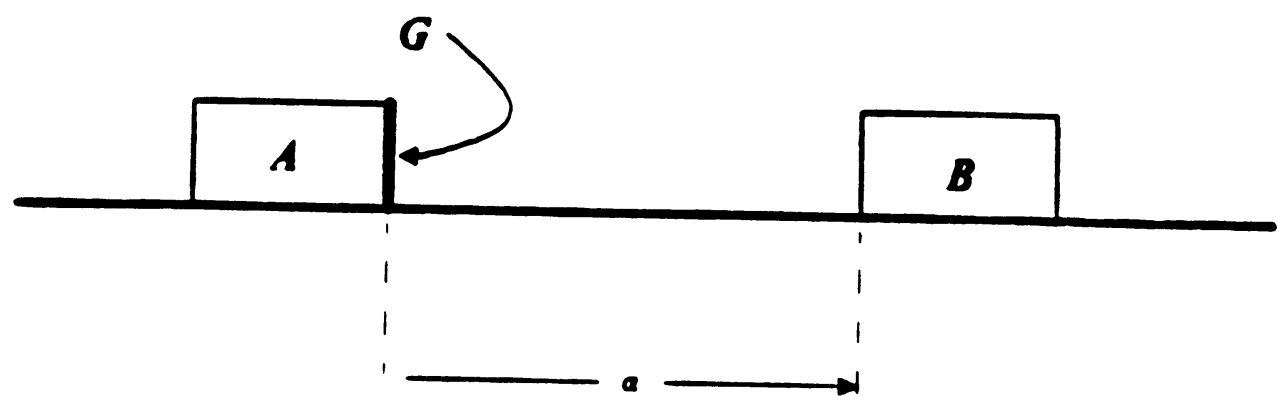

Figure 15: The equivalent problem in configuration space. The blocks $A$ and $B$, the distance between the blocks $a$, and the commanded velocity $v_{0}=v_{0}^{*}$ with control error cone $B_{e c}\left(v_{0}^{*}\right)$. The position of $A$ is fixed. 


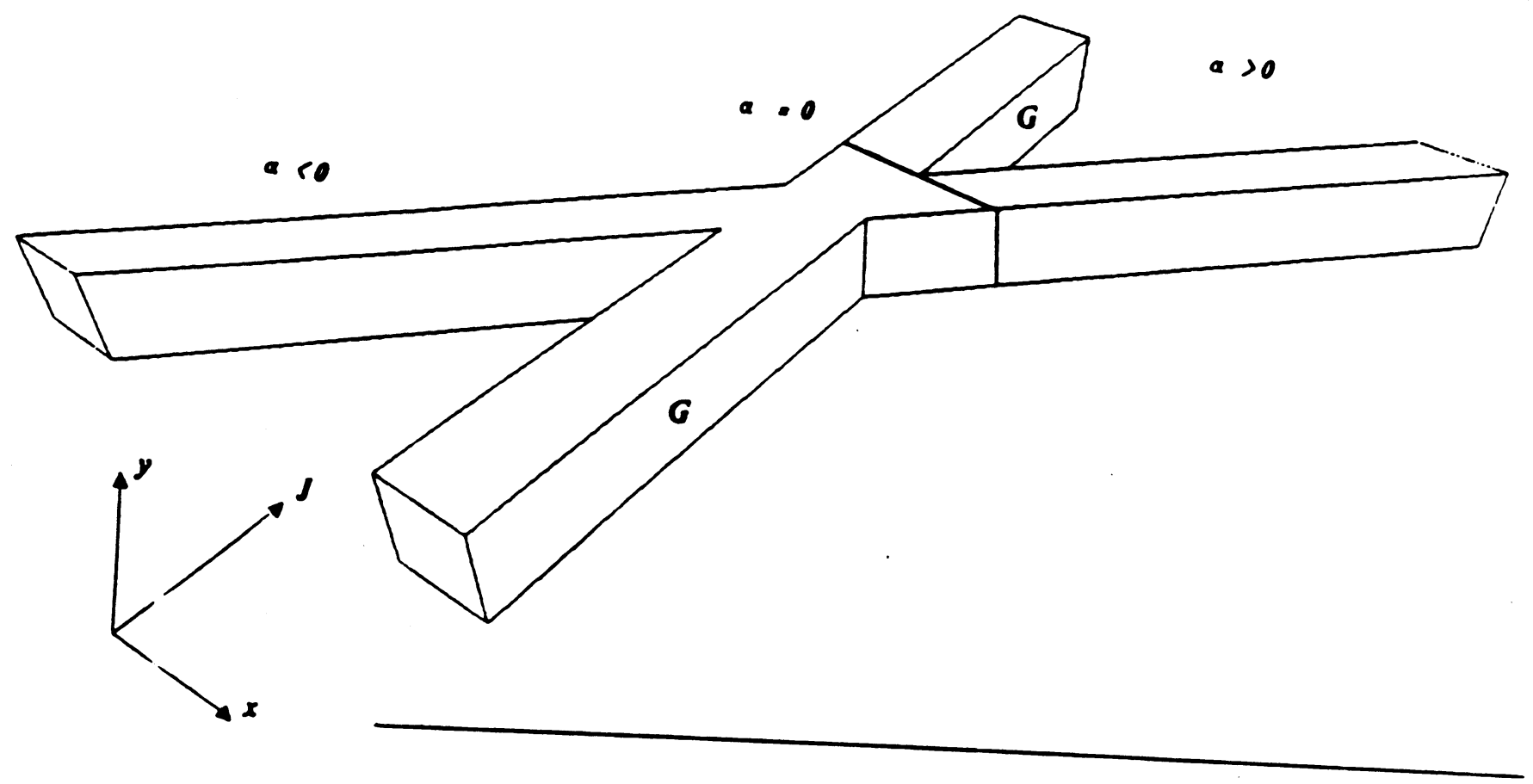

Figure 16: The generalised configuration space obstacles for example (1). The generalised configuration space is three dimensional, having and $y$ degrees of motion freedom, and an a degree of model error freedom. Legal motions are parallel to the $z-y$ plane, and orthogonal to the $J$ axis. 
15. This cone lies in the phase-space for $C^{\prime}$, denoted $T C^{\prime}$. (Phase space is simply Position-space $\times$ Velocity-space. A point in phase space has the form $(x, v)$, and denotes an instantaneous velocity of $v$ at configuration $x$ ). Phase space represents all possible velocities at all points in $C^{\prime}$. The phase space for $\mathcal{G}$ is obtained by indexing $T C$ by $J$ to obtain $T C \times J$. All velocities in generalized configuration space lie in $T C \times J$. For Ex. (1) $T C \times J$ is $\Re^{4} \times\left[-d_{0}, d_{0} \mid\right.$. The generalized velocity uncertainty cones are two-dimensional, parallel to the $x$-y plane, and orthogonal to the $J$ axis.

4. Generalized damper dynamics extend straight-forwardly to $\mathcal{G}$, so motions satisfy $f=B\left(v-v_{0}\right)$ where $f, v$, and $v_{0}$ lie in $T C \times J$. Thus friction cones from configuration space (see [Erdmann]) naturally embed like generalized velucity cones in $T C^{\prime} \times J$.

These axioms give an intuitive description of the physics of $\mathcal{G}$. A formal axiomatization is given in [Donald 86, 87]. We have captured the physics of $\mathcal{G}$ using a set of generalized uncertainties, friction, and control characteristics (1-4). These axioms completely characterize the behavior of motions in $\mathcal{G}$.

\subsubsection{Pushing}

By relaxing axiom (1), above, we can consider a generalization of the model error framework, in which pushing motions are permitted, as well as compliant and gross motions. We relax the assumption that motion between universes is impossible, and permit certain motions across $J$. Consider example (3). Observe that a displacement in $J$ corresponds to a displacement in the position of the block $B$. Thus a motion in $J$ should correspond to a motion of $B$. Suppose the robot can change the position of $B$ by pushing on it, that is, by exerting a force on the surface of $B$. The key point is that pushing operations may be modeled by observing that commanded forces to the robot may result in changes in the environment. That is, a commanded force to the robot can result in motion in $C$ (sliding) as well as motion in $J$ (pushing the block). Let us develop this notion further.

Our previous discussion assumed that motion across $J$ was impossible. That is, all motion is confined to one $\alpha$-slice of generalized configuration space. In example (3), this is equivalent to the axiom that $B$ does not move or deform under an applied force. Such an axiom makes sense for applications where $B$ is indeed immovable, for example, if $A$ and $B$ are machined tabs of a connected metal part. However, suppose that $B$ is a block that can slide on the table. Then an applied force on the surface of the block can cause the block to slide. This corresponds to motion in $J$. In general, the effect of an applied force will be a motion which slides or sticks on the surface of $B$, and which causes $B$ to slide or stick on the table. This corresponds to a coupled motion in both $C$ and $J$, that is, a motion across $\alpha$-slices of generalized configuration space. Such a motion is always tangent to a surface in generalized configuration space. 
In [D], we generalize the description of the physics of $\mathcal{G}$ to permit a rigorous account of such motions. This model can then be employed by an automated planner. Such a planner can construct motion strategies whose primitives are gross motions, compliant motions, and pushing motions. This model of pushing is used in the gear-meshing example, where a model error parameter-the orientation of $B$-can be changed due to pushing.

\subsection{Guaranteed Plans}

A motion strategy [LMT) is a commanded velocity (such as $v_{0}^{*}$ in fig. 15) together with a termination predicate which monitors the sensors and decides when the motion has achieved the goal. Given a goal $G$ in configuration space, we can form its preimage [LMT]. The preimage of $G$ is the region in configuration space from which all motions are guaranteed to move into $G$ in such a way that the entry is recognizable. That is, the preimage is the set of all positions from which all possible trajectories consistent with the control uncertainty are guaranteed to reach $G$ recognizably. For example, see fig. 17. The entry is recognized by monitoring the position and velocity sensors until the goal is attained. Fig. 17 is a directional preimage: only one commanded velocity $v_{\theta}^{*}$ is considered. Here all preimage points reach the goal recognizably under this particular $v_{\boldsymbol{\theta}}^{*}$. The non-directional preimage is the union of all directional preimages.

We envision a back-chaining planner which recursively computes pre-images of a goal region. Successive subgoals are attained by motion strategies. Each motion terminates when all sensor interpretations indicate that the robot must be within the subgoal. [LMT,E] provide a formal framework for computing preimages where there is sensing and control uncertainty, but no model error. In particular, [Erdmann) shows how backprojections may be used to approximate preimages. The backprojection of a goal $G$ (with respect to a commanded velocity $v_{0}^{*}$ ) consists of those positions guaranteed to enter the goal (under $v_{\theta}^{*}$ ). Recognizability of the entry plays no role. Fig. 18 illustrates the difference between backprojections and preimages. Here the radius of position sensing uncertainty is greater than twice the diameter of the hole. Sliding occurs on all surfaces. Furthermore, we assume that the robot has no sense of time (i.e., no clock)-for example, it might be equipped with a contact sensor that only fires once. The back projection $B_{\theta}(G)$ strictly contains the preimage $P_{0}(G)$ : while all points in the backprojection are guaranteed to reach $G$, the sensing inaccuracy is so large that the termination predicate cannot tell whether the goal or the left horizontal surface has been reached. Only from the preimage can entry into $G$ be recognized.

Preimages provide a way to construct guaranteed plans for the situation with no model error. Can preimages and backprojections be generalized to situations with model error? The answer is yes. The generalized control and sensing uncertainties in $\mathcal{G}$ are given by the physics axioms above. These uncertainties completely determine how motions in generalized configuration space must behave. We form the backprojection of $G$ under 


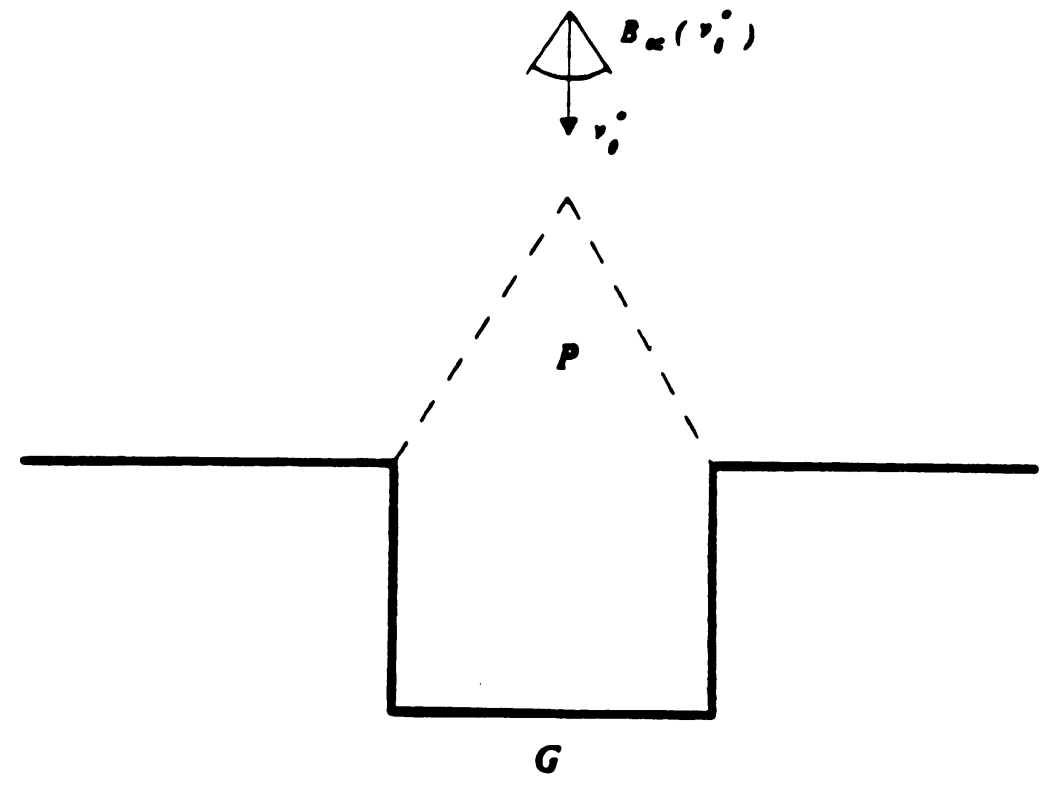

Figure 17: The goal is the region $G$. Sliding occurs on vertical surfaces, and sticking on horizontal ones. The commanded velocity is $v_{j}^{*}$, and the control uncertainty is $B_{e c}\left(v_{j}^{*}\right)$. The presmage of the $G$ with respect to $\theta$ is the region $P$. 


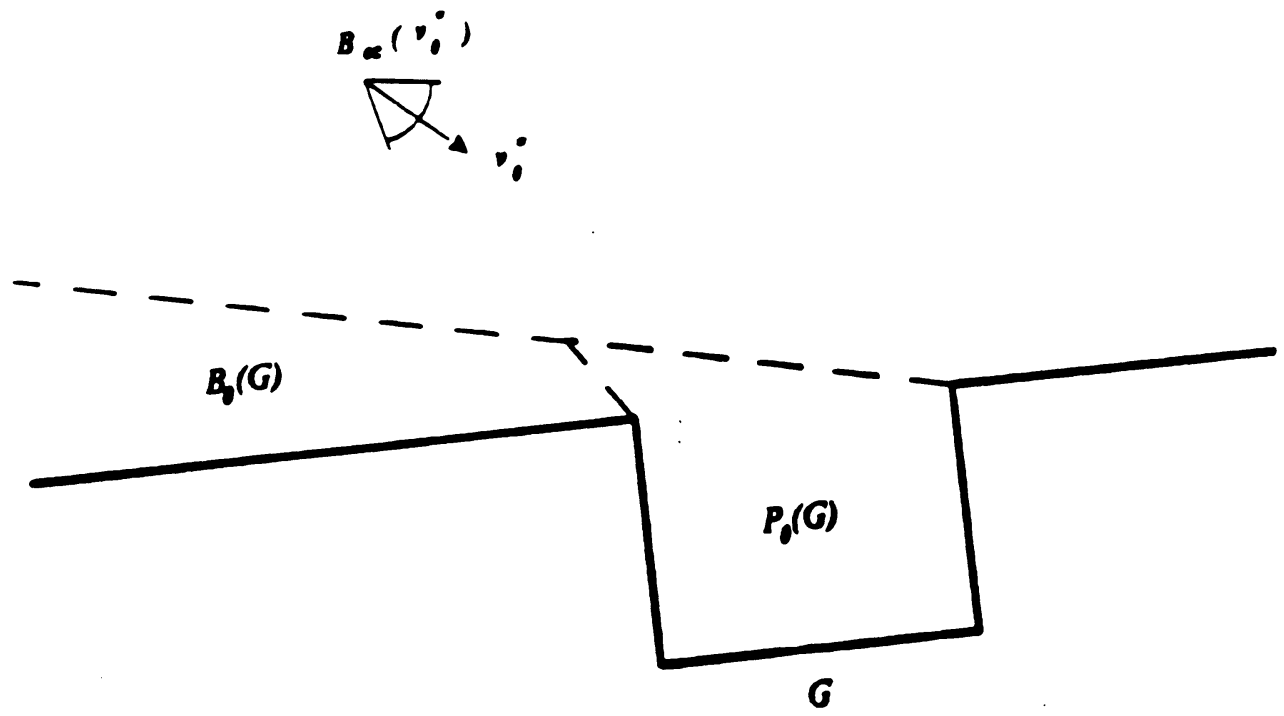

Figure 18: Here, the radius of the position sensing uncertainty ball is twice the width of the hole. Sliding occurs on all surfaces under the control velocities shown. The preimage of the goal under commanded velocity $v_{\theta}^{*}$ is $P_{0}(G)$. The backprojection $B_{0}(G)$ strictly contains this preimage: while all points in the backprojection are guaranteed to reach $G$, the sensing inaccuracy is so large that the termination predicate cannot tell whether the goal or the left horizontal surface has been reached. Only from the preimage can entry into $G$ be recognised. 
these uncertainties. The trick here is to view the motion planning problem with $n$ degrees of motion freedorn and $k$ degrees of model error freedom as a planning problem in an $(n+k)$-dimensional generalized configuration space, endowed with the special physics described above. The physics is characterized precisely by axioms defining certain special sensing and control uncertainties in $\mathcal{G}$. The definitions and results for pre-images and backprojections $|\mathrm{LMT}, \mathrm{E}|$ in configuration space generalize mutatis mutandis to $\mathcal{G}$ endowed with this physics; this is proved in [D]. Thus our framework reduces the problem of constructing guaranteed motion strategies with model error to computing preimages in a somewhat more complicated, and higher-dimensional configuration space. For details, see [Donald 86, 87, 88].

\subsection{Error Detection and Recovery}

If we were exclusively interested in constructing guaranteed motion strategies in the presence of model error, we would be done defining the framework: having reduced the problem to computing preimages in $\mathcal{G}$, we could now turn to the important and difficult problems of computing and constructing $\mathcal{G}$, and further extend the work of [LMT,E] on computing preimages in general configuration spaces.

However, guaranteed strategies do not always exist. In example (3), (figs. 14-16) there is no guaranteed strategy for achieving the goal, since the goal may vanish for some values of $\alpha$. Because tolerances may cause gross topological changes in configuration space, this problem is particularly prevalent in the presence of model error. In the pegin-hole problem with model error (figs. 3-13) the goal may also vanish (the hole may close up) for certain regions in $J$. More generally, there may be values of $\alpha$ for which the goal may still exist, but it may not be reachable. For example, in a variant of the problem in fig. 3, an obstacle could block the channel to the goal. Then $G$ is non-empty, but also not reachable. Finally, and most generally, there may be values of $\alpha$ for which the goal is reachable but not recognizably reachable. In this case we still cannot guarantee plans, since a planner cannot know when they have succeeded.

These problems may occur even in the absence of model error. However, without model error a guaranteed plan is often obtainable by back-chaining and adding more steps to the plan. In the presence of model error this technique frequently fails: in example (1), no chain of recursively-computed preimages can ever cover the start region $R \times J$. The failure is due to the peculiar sensing and control characteristics (1-4) in generalized configuration space .

In response, we will develop Error Detection and Recovery (EDR) strategies. These are characterized as follows:

- An EDR strategy should attain the goal when it is recognizably reachable, and signal failure when it is not. 
- It should also permit serendipitous achievement of the goal.

- Furthermore, no motion guaranteed to terminate recognizably in the goal should ever be prematurely terminated as a failure.

- Finally, no motion should be terminated as a failure while there is any chance that it might serendipitously achieve the goal due to fortuitous sensing and control events.

These are called the "EDR Axioms", they will be our guiding principles. We now state how such EDR strategies may be constructed; for proofs and more detail, see [Donald $86,87,88 \mid$.

Suppose that a planning prublem is given with two disjoint geometrical goals, $G_{1}$ and $G_{2}$. We may insist that the run-time executor be able to terminate the strategy and also be able to disambiguate which goal has been reached. In this case, we can construct the preimage of the distinguishable union of $G_{1}$ and $G_{2}$, which we write as

$$
P_{\theta}\left(\left\{G_{1}, G_{2}\right\}\right) \text {. }
$$

If $\theta$ is executed starting in this preimage, then the motion can always be recognizably and distinguishably terminated in either $G_{1}$ or $G_{2}$.

We can characterize EDR strategies geometrically as follows. Suppose the geometric goal is $G$. The implicit "meaning" of $G$ is: recognizably achieving $G$ is equivalent to "success." We introduce an "additional" goal-like set $H$, which is disjoint and distinguishable from $G$, such that when $H$ is recognizably achieved, then failure of the motion may be signalled. That is, we construct an $H$ such that recognizably achieving $H$ is equivalent to "failure." $H$ is called the $E D R$ region. Remarkably, $H$ may be selected such that the EDR axioms are satisfied. In [Donald $86,87,88$ ], we derive $H$ as follows. (iiven a motion $\theta$ and a start region $R$, first we define $H$ using reachability constructs only. Then we test whether $H$ and $G$ are distinguishable using sensors (this is the "formal test" alluded to in the prelude). If so, then by the construction of $H$, we have

$$
R \subset P_{\theta}(\{G, H\}) .
$$

Furthermore, using $H, \theta$ is a one-step EDR strategy satisfying the EDR axioms. Here is an idea of what $H$ is like: In fig. 19 the EDR region $H$ is shown (in position space only) fur example (3). Consider $H$ as a two-dimensional region in $\mathcal{G}$; just a slice of it is shown in fig. 19. Note that in this example, $H$ only exists in the slices in which $G$ vanishes. Here, given the sensing uncertainty bounds of example (3), the termination predicate can distinguish between $G$ and $H$ based on position sensing, velocity sensing, or elapsed time. Clearly, $H$ satisfies the EDR axioms: the motion is guaranteed to terminate recognizably in $G$ iff the motion began in a universe in which $G$ does not vanish. Otherwise, the motion terminates recognizably in $H$. In the first case, the termination predicate signals success, in the latter, failure. 




Figure 19: A typical $\alpha$-slice of the EDR region $H$, for $\alpha$ small and negative. The goal vanishes in this slice; the dashed line indicates where the goal would be in other slices. Points in $H$ lie within the forward projection (since they are reachable), yet outside the weak-preimage (since the goal is unachievable). 
Here is how we construct $H$. The forward projection of a set $R$ under $\theta$ is all configurations $^{\nabla}$ which are possibly reachable from $R$ under $v_{\theta}^{*}$ (subject to control uncertainty). It is denoted $F_{\theta}(R)$. Forward projections only address reachability: the termination predicate is ignored and only the control uncertainty bound and commanded velocity $v_{\theta}^{*}$ are needed to specify the forward projection. See figs. 8, 9, 1112 .

So far the preimages we have considered are strong preimages, in that all possible motions are guaranteed to terminate recognizably in the goal. The weak preimage [LMT] (with respect to a commanded velocity) is the set of points which could possibly enter the goal recognizably, given fortuitous sensing and control events. See fig. 20. We will use the weak preimage to capture the notion of serendipity in the EDR axions. The idea is that a motion may be terminated in failure as soon as egress from the weak preimage is recognized. The weak preimage is denoted $\hat{P}_{\theta}(G)$.

We define $H_{0}$ to be the set difference of the forward projection minus the weak preimage:

$$
H_{0}=F_{\theta}(R)-\hat{P}_{\theta}(G)
$$

Clearly, the motion $\theta$ can be terminated as a failure whenever $H_{0}$ has been reached, since $H_{0}$ is outside the weak preimage; hence the goal cannot be attained under $\theta$ from there.

We also define $H$, to be all regions where sticking is possible in the weak minus strong preimage:

$$
H_{s}=\left\{x \in \hat{P}_{\theta}(G)-P_{\theta}(G) \mid \text { sticking is possible at } x\right\} .
$$

See fig. 21. The motion $\theta$ should also be terminated as a failure if sticking occurs in $H_{s}$. We will decree that the robot has stuck in $H_{s}$ if its velocity is zero for some duration, or "time-out" period. More precisely, we define $H$ to be a set in phase-space. First, note that $H$ contains all phase-space points $(x, v)$ where $x$ is in $H_{0}$. Second, $H$ contains points of the the form $(x, 0)$, where $x$ is in $H_{.}$. Thus, viewing phase-space as the tangent bundle to generalized configuration space, $H$ contains the "cylinder" $\pi^{-1}\left(H_{0}\right)$ of velocities over $H_{0}$, and the "zero section" $Z\left(H_{0}\right)$ of zero velocities over $H_{s}$ :

$$
H=\pi^{-1}\left(H_{0}\right) \cup Z\left(H_{s}\right) .
$$

This definition of $H$ almost satisfies the EDR axioms-the only tricky point is that we cannot guarantee that after sticking in $H$, for a long time, the robot cannot eventually slide into the goal. This may be handled in principle by introducing a time-out period by which the goal must be reached. That is, our definition of $H$ satisfies the EDR axioms if

\footnotetext{
${ }^{8}$ Actually, forward projections are in phase-space, so this is the position component of the forward projection.
} 


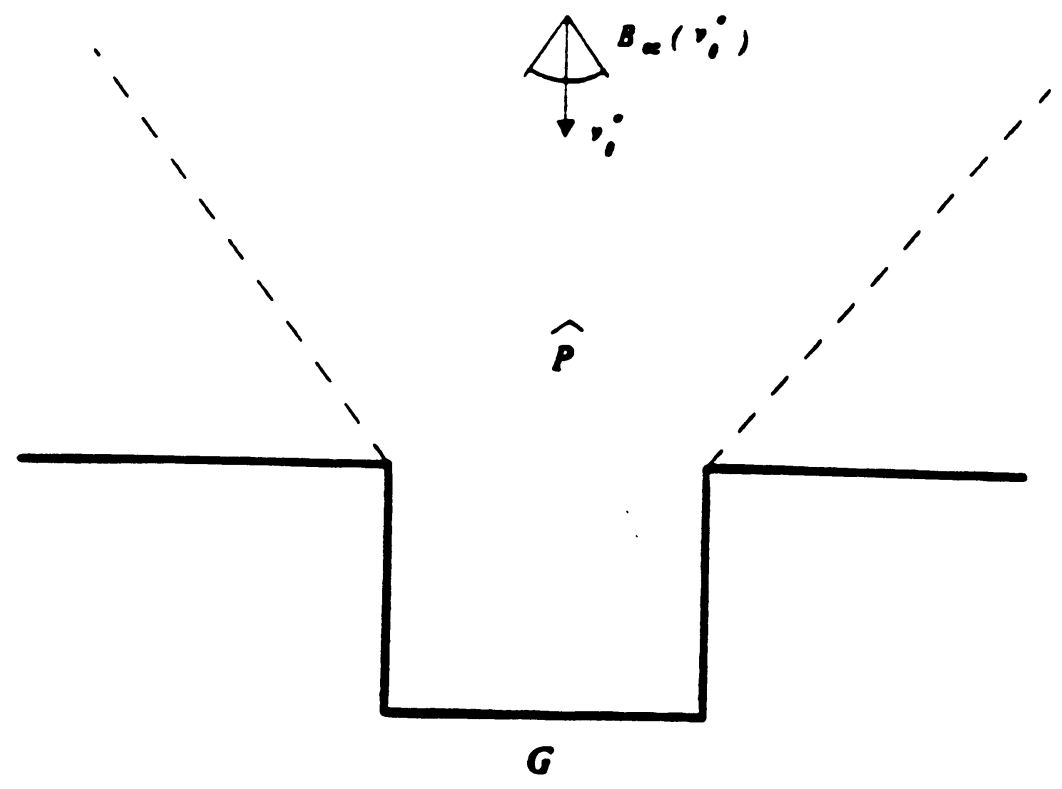

Figure 20: The weak preimage of the goal $G$ under $v_{\dot{0}}^{*}$. Compare fig. 17.

Figure 21: $H_{0}$ in eq. (1) is not the entire EDR region. Sticking may occur within the weak preimage

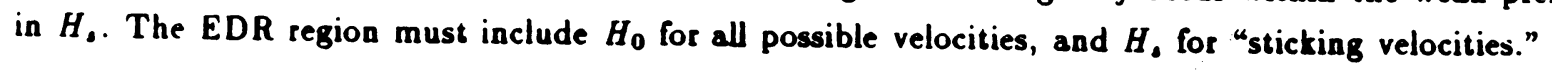




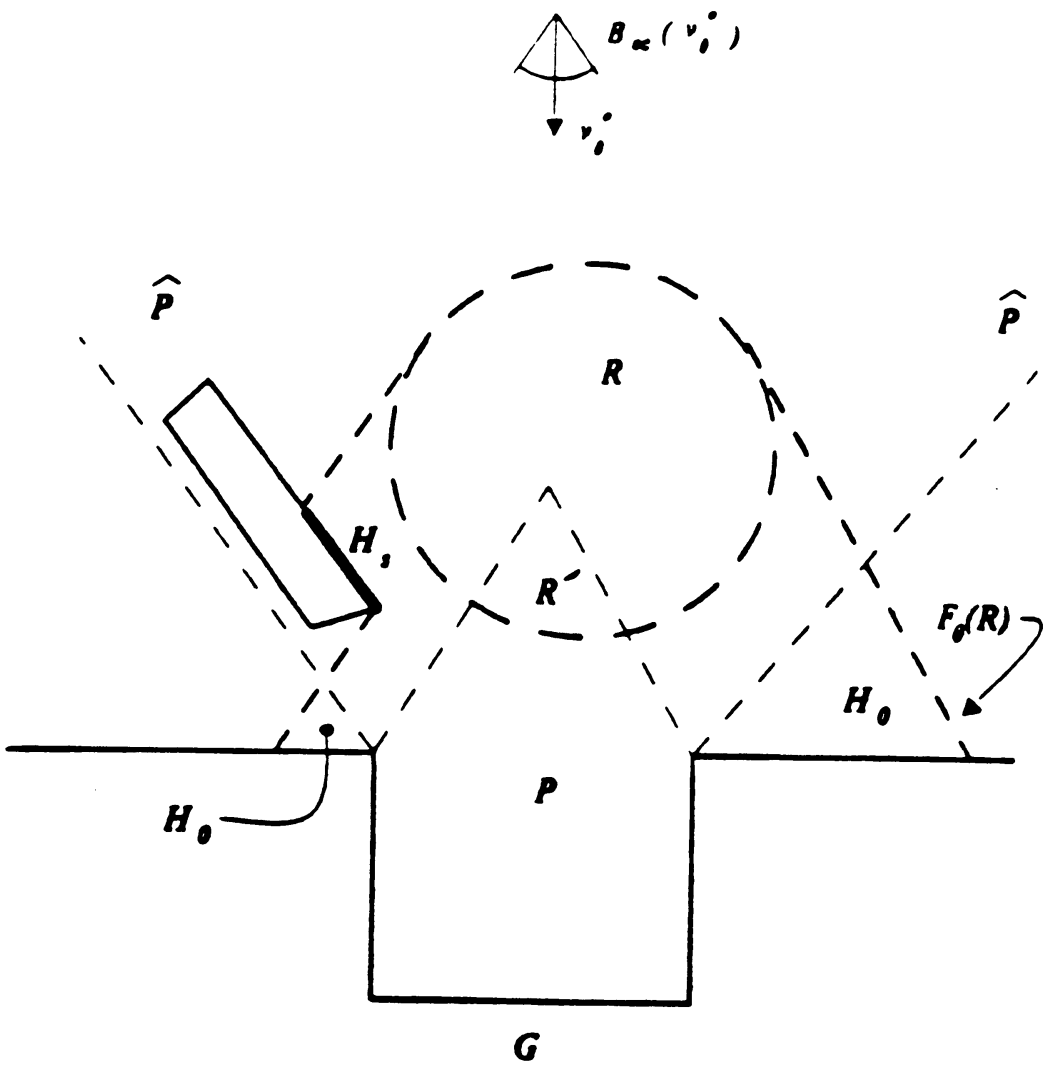

Figure 21. $H_{0}$ is not the entire EDR region. Sticking may occur within the weak preimage in $H_{.}$. The EDR region must include $H_{n}$ for all possible velocities, and $H$, for "sticking velocities." 
the goal is specified in phase-space-time as the product of $\pi^{-1}(G)$ with a compact time interval.

In $|\mathrm{D}|$, we showed how to compute $H$ in the domain of planar assemblies with model error--a domain which included both the gear-meshing example (fig. 2) and the peg-inhole problem with model error (figs. 3-13). We also showed how to compute whether $\boldsymbol{G}$ and $H$ are distinguishable. This is sufficient to generate one-step EDR strategies. These algorithms have been implemented in LIMITED. At a high level, the one-step algorithm is:

Algorithm IEDR

1. Generate a commanded velocity $v_{\theta}^{*}$.

2. Compute the $E D R$ region $H$ for $v_{\theta}^{*}$,

3. Determine whether the $E D R$ region $H$ and the goal $G$ are distinguishable using sensors. If so, then $v_{\theta}^{*}$ yields a one-step EDR strategy which recognizably terminates in $G$ or $H$ by monitoring position and force sensors.

4. Let $\operatorname{push}_{\theta}(G)$ and $\operatorname{push}_{\theta}(H)$ denote the sticking push-forwards. They are the set of obslacle edges within $G$ and $H$, resp., on which sticking can occur under $v_{\theta}^{*}$. Determine whether these regions are distinguishable using sensors. If so, then $v_{\theta}^{*}$ yields a one-step EDR strategy which recognizably terminates when sticking is detected. sors?"

Here is how Limited decides the question, "Are $G$ and $H$ distinguishable using sen-

$H$ and $G$ are distinguishable using position sensing alone if their convolutions (Minkowski sums) by the position sensing error ball $B_{\text {ep }}$ do not intersect.

Each obstacle edge of $H$ and $G$ has an associated configuration space friction cone. Two edges are distinguishable using force sensing if the convolutions of their friction cones by the force sensing uncertainty $B_{\text {ev }}$ have a trivial intersection. ${ }^{9}$

Similarly, the set of possible sensed reaction forces at an obstacle vertex $w$ of $G$ or $H$ may be found by taking the direct sum of the friction cones of the edges cobounding $w$, and convolving by $B_{\text {ev }}$. Again, a vertex of $H$ and a vertex (or edge) of $G$ are distinguishable using force sensing if their associated cones of sensed reaction forces have a trivial intersection.

The procedure also works for determining the distinguishability of the push-forwards. Note that the procedure is correct for linear edges, where position- and force-sensing are separable because the set of possible reaction forces is constant along an edge. For the general case, see [Donald, 87].

However, for both the gear-meshing and peg-in-hole problem, one-step strategies cannot be generated for the sensing and control error bounds we input. More specifically:

\footnotetext{
${ }^{9} \mathrm{An}$ intersection containing only the zero-vector.
} 
in these cases, $H$ can be computed for any hypothesized one-step motion $\theta$, but LIMITED cannot find a $\theta$ for which $H$ is distinguishable from the goal. Intuitively, the reason that $H$ and $G$ are not distinguishable is that (a) since the control uncertainty is "large", therefore $H$ is "large", and (b) since the error bounds on position and force sensing are large, therefore it is not always possible to distinguish between $H$ and $G$.

What is to be done in this case? In short: multi-step strategies are required. This raises the central issue: how can multi-step EDR strategies be generated? We are interested both in an implementable (and, in LIMITED, implemented) practical approach, and also in developing unifying theory for multi-step EDR planning.

In this paper, we will assume that $H$ can be computed given $R$ and $\theta$, and that we can test whether or not it is distinguishable from $G$. The reader interested in algorithms for these computations may find details in [Donald $86,87,88$ ]. We now turn our attention to the generation of multi-step EDR strategies, given these tools.

\subsection{The Preimage Structure of EDR Regions}

Our notation for preimages must be made slightly more formal for the sequel. The key point [LMT,E,Mason] is that knowing where the motion began (that is, knowing $R$ ) can be used by the termination predicate to predict what configurations are reachable, and therefore, to effect recognizable termination. This prediction may be accomplished via the forward projection; its use as such was termed history by Erdmann. Thus, the preimage map $P$ depends not only on $\theta$ and $G$, but also on $R$. Our notation for preimages must reflect this dependence.

We observed above that if the termination predicate can distinguish between the goal $G$ and the EDR region $H$, then $H$ is a good EDR region and an EDR strategy was in hand. Formally, we write this recognizability constraint as

$$
P_{\theta, R}(\{G, H\})=R .
$$

We say that the preimage $(*)$ is taken with respect to $R .(*)$ means that the (strong) preimage of the set of goals $\{G, H\}$, with respect to commanded velocity $v_{\theta}^{*}$, is all of $R$. When we have a set of goals, the termination predicate must return which goal ( $G$ or $H$ ) has been achieved. This is different from $P_{0, R}(G \cup H)$, which means the termination predicate will halt saying "we've terminated in $G$ or $H$, but I don't know which." The region $R$ appears on both sides of (*) because the preimage depends on knowing where the motion started. This is a subtle point, see [LMT,E]. Thus solving preimage equations like (*) for $R$ is like finding the fixed point of a recursive equation. Here, however, we know $R, H$, and $G$, so (*) is a constraint which must be true, rather than an equation to solve. Presumably $(*)$ is easier to check than to solve for $R$; see [LMT,E, D]. 


\subsubsection{The Most General Preimage Equation}

We now introduce the most general form of the preimage equation. Suppose $\left\{G_{\beta}\right\}$ denotes a collection of goals, and $\left\{R_{\alpha}\right\}$ is a collection of start regions. Recall $\theta$ denotes the direction of the commanded motion. Most generally, the preimage equation is

$$
P_{\theta,\left\{R_{\alpha}\right\}}\left(\left\{G_{\beta}\right\}\right)=\left\{R_{\alpha}\right\} .
$$

This says that if the run-time executor knows that the robot is in some particular but arbitrary start region $R$ in the collection $\left\{R_{\alpha}\right\}$, then if velocity $v_{j}^{*}$ is commanded, then the termination predicate is guaranteed to achieve some goal $G$ in $\left\{G_{\beta}\right\}$, and, furthermore, it can recognize which goal has been achieved.

\section{Multi-Step Strategies}

In this section we explure multi-step strategy construction. Now, in principle, having reduced both model error and EDR to essentially "preimage-theoretic" equations, multistep strategies could be synthesized by solving these preimage equations. While this is proved or at least implicit in previous work [LMT,Mason,E,D], it is far from obvious; furthermore, there are almust no published examples of such strategies. For this reason we begin by presenting a worked-out example of a motion plan using preimages. The motion problem is grasp-centering for a robot gripper in the presence of model error. Both guaranteed and EDR strategies are found by solving the preimage equations.

Preimages are a key underlying tool for the geometric EDR theory, and the [LMT] framework is in some sense a "universal" method for synthesizing multi-step strategies. However, the technique of solving the preimage equations is not computational. For this reason, we introduce a construction called the push-forward. Roughly speaking, the push-forward is that subset of the forward projection where the inotion can terminate. Since push-forwards address termination whereas forward projections do not, we may regard them as "dual" to preimages. That is, push-forwards are to forward projections as preimages are to backprojections. Second, the push-forward permits us to develop rather simple algorithms for planning multi-step strategies. These algorithms have been implemented in LimiTED. While the push-forward method for multi-step strategy synthesis is algorithmic, it is less general than the full preimage method (solving the preimage equations). We characterize the loss of power in push-forward algorithms.

In section 1 we presented two EDR plans generated by LiMITED. These were the peg-inhule insertion strategy with model error, and the gear-meshing plan. Both were two-step plans. We will go into more detail in describing how these plans were generated. The peg-in-hole plan used push-forward techniques. The gear plan used a seemingly unrelated 
technique called failure mode analysis. We describe failure mode analysis and algorithms for computing it.

Next, we will present a view of multi-step strategies which essentially unifies all these techniques. This is called the "weak" EDR theory. The motivation behind this theory is that when a motion terminates ambiguously, a subsequent motion may be synthesized which disambiguates the success or failure of the first. Oddly enough, it is not necessary for either motion individually to satisfy the EDR axioms. However, when taken together, the two-motion plan can often be considered "equivalent" to a one-step EDR strategy.

The weak EDR theory effectively defines some laws of "compusition" that permit two single-step plans to be concatenated into a two-step plan satisfying the EDR axioms. Hence it is often possible to construct multi-step plans that are EDR plans "globally" although not "locally". That is, considered as entire plans, they satisfy the EDR axioms; this is the "global" condition. However, "locally" they are not EDR plans, in that no single step is an EDR strategy. The key to pasting together non-EDR plans to make a global EDR strategy lies in defining certain local "niceness" conditions for how plans must mesh. These are called the linking conditions.

Starred sections may be skipped if desired at first reading.

\section{Planning using Preimages: A Detailed Example}

In this section we show how the [LMT] framework can be used to synthesize multi-step strategies. Here are the key points of this section:

- In principle, multi-step plans may be found by solving a family of preimage equations.

- While this was proved by [LMT,Mason,E], it is not obvious how to effect the solution. This example intends to elucidate the process.

- The technique is general enough to plan EDR strategies under model error, once we have cast both the problem of planning with model error and the EDR problem in an essentially "preimage-theoretic" form, as in [D] and section 1.

- However, the technique of solving the preimage equations is not algorithmic.

Furthermore, preimages are a key underlying tool for the geometric EDR theory. It is necessary to make further acquaintance with preimages in order to continue our development of the EDR framework. To that end, this section presents a worked-out example of a motion plan using preimages. The motion problem is grasp-centering for a robot gripper in the presence of model error. The example illustrates the use of the 
preimage framework to derive a multi-step motion strategy in the presence of model error. The strategy employs time-sensing and force-sensing. This discussion is designed both as a tutorial in solving preimage equations for a motion plan, and as an introduction to the planning of multi-step strategies.

\subsection{Example: Planning Grasp-Centering using Preimages}

The remainder of this paper builds on the preimage framework to develop the EDR theory. To make the framework more accessible, we provide here a fairly detailed description of a motion planning problem using preimages. ${ }^{10}$

We are now ready to work an example. We solve a particular motion planning problem with model error by solving the preimage equations. This example provides an illustration of planning using preimages. For simplicity, we initially address only the problem of finding a guaranteed strategy. Finding EDR strategies in this domain is discussed afterwards.

Consider the grasp-centering problem shown in fig. 22. The task is to center the robot gripper over the block $D$. The gripper can translate but not rotate in the plane. In its start position, the gripper is somewhere over $D$, such that the bottom of the fingers $F A$ and $F B$ are below the top of $D$. The width of $D$ is unknown, but must be less than the distance between $F A$ and $F B$. We assume $D$ is fixed (it cannot be accidentally pushed).

Hence we can regard this as a planning problem with model error. $C$ is taken to be the cartesian plane, and $J$ is a bounded interval of the positive reals. Our first question is, what does the generalized configuration space look like? This is easily answered by considering the motion planning problem in fig. 23. The problem is to find a motion strategy for a point robot so that it can achieve a goal exactly halfway between the blocks $A$ and $B$. The distance $\alpha$ between $A$ and $B$ is unknown and positive. The point robot is known to start between $A$ and $B$. Again, the point can translate in the plane. The distance $\alpha$ is the model error parameter. It is easy to see that the problems in figs. 22 and 23 are equivalent.

However, we already know what the generalized configuration space for fig. 23 looks like. It was discussed in section 1 , and is shown in fig. 16. Hence our example is a planning problem in a familiar generalized configuration space.

Next, we assume that the robot has perfect control, perfect velocity sensing, and a perfectly accurate sense of time. However, it has infinite position sensing error. 11

Now, since the gripper starts over $D$ with the bottom of the fingers below the top of $D$, and since the robot has perfect control, it suffices to consider the $x$ axis of $C$. Since

\footnotetext{
${ }^{10}$ This problem arose in discussions with Tomás Lozano-Pérez, John Canny, and Mike Erdmann.

11 This example is easily generalized to non-zero control, time-sensing, and force-sensing error, and finite position-sensing error. This requires giving the goal non-empty interior, however.
} 


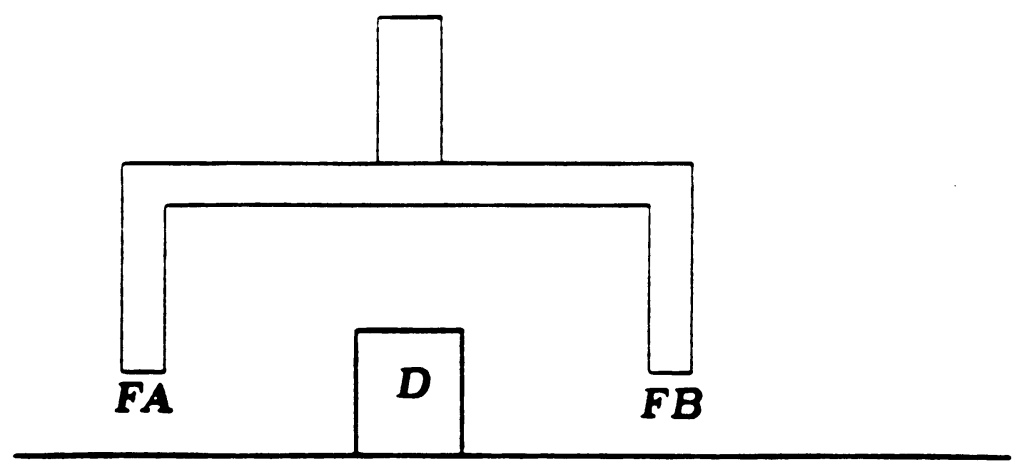

Figure 22: The grasp centering problem. The width of the block $D$ on the table, and the position of the gripper are only known approximately. 


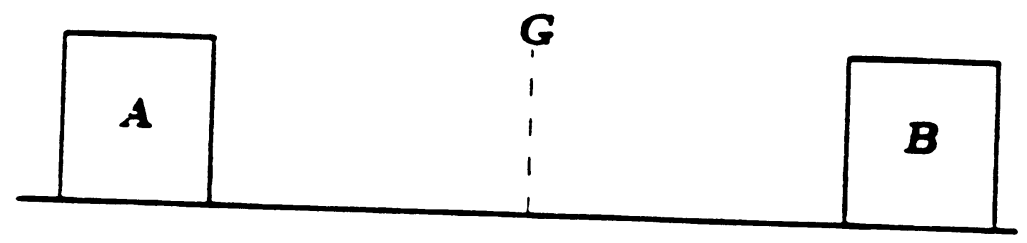

Figure 23: An equivalent problem. A point robot must be navigated halfway between the blocks $A$ and $B$. The distance between $A$ and $B$ is not known. The robot has force sensing, and a clock. However, it has poor position sensing. We regard $C$ as $\Re^{2}$ and $J$ as the bounded interval $(0, d \mid$ for $d$ positive. The generalized configuration space for this problem is the same as in fig. 16, for the positive values in $J$. 


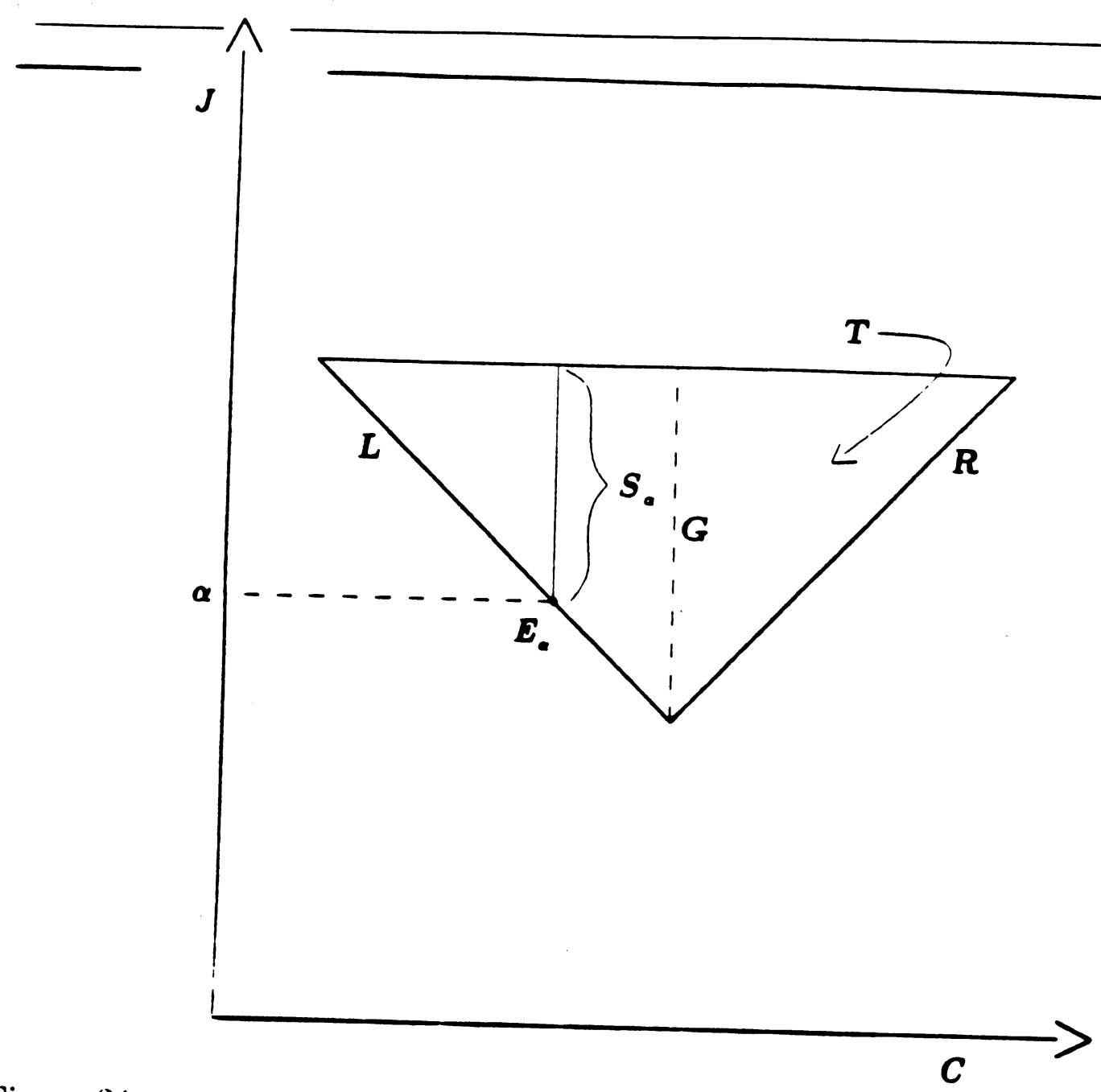

Figure 24: Assuming that the gripper fingers are initially lower than the top of the block $D$, the $y$ dimension can effectively be ignored. This allows us to examine a cross-section of fig. 16 . We treat $C$ as the axis of motion freedom, yielding a 2D $C \times J$ planning space. $L$ and $R$ are obstacle boundaries in generalised configuration space. The goal is the bisector $G$ between $L$ and $R$ in free-space. The start region $T$ is the triangular region between $L$ and $R$. $E_{\alpha}$ is a point on $L$. $S_{\alpha}$ is a line in $T$ parallel to $G$ and containing $S_{a}$. 
the $y$ axis can be ignored, we develop our example in the plane, that is, in the generalized configuration space where $C$ and $J$ are both one-dimensional. This $2 \mathrm{D}$ generalized configuration space is shown in fig. 24 , which is essentially an $x-J$ cross-section of fig. 16, holding $y$ constant with $\alpha$ constrained to be positive. In fig. $24, L$ and $R$ are left and right obstacle edge boundaries generated by $A$ and $B$. The goal is the line in free-space bisecting $L$ and $R$. The start region $T$ is the triangular region in free-space between $L$ and $R$. ( $T$ is the convex hull of $L$ and $R$ ).

Now, since motion across $J$ is not permitted, all motions are parallel to the $x$ axis, that is to say, horizontal in fig. 24. There are only two kinds of motions the planner can command. Let + denote a motion to the right, and - a motion to the left. We assume the robot has perfect control over the magnitude as well as the direction of the commanded velocity.

See fig. 24. Now, if $\alpha$ is a point on the $J$ axis, let $E_{\alpha}$ be the point on the left obstacle edge $L$ with $J$ coordinate $\alpha$. We will denote the collection of all such points on $L$ by $\left\{E_{\alpha}\right\}$. Let $S_{\alpha}$ denote the maximal line segment within $T$ containing $E_{\alpha}$ and parallel to $G$. Formally, if $E_{\alpha}$ has coordinates $(x, \alpha)$, then $S_{\alpha}$ is the line segment extending from $E_{\alpha}$ to $(x, d)$ where $d$ is an upper bound on the distance between $A$ and $B$. We denote the collection of all lines $S_{\alpha}$ by $\left\{S_{\alpha}\right\}$.

At this point we are prepared to derive a motion strategy for centering the grasp, that is, for attaining $G$ from $T$. The strategy has three steps. The termination conditions for the motions involve time- and force-sensing. Here is the motion strategy in qualitative terms:

\section{Strategy Guarantee-Center}

1. Command a motion to the right. Terminate on the right edge $R$ based on force sensing.

2. Command a velocity of known magnitude to the left. Terminate when in contact with the left edge L, using force sensing. Measure the elapsed time of the motion. Compute the distance traversed. This gives exact knowledge of where the motion terminated on $L$. The effect of this step is to measure the distance $\alpha$ between the blocks.

\section{Move distance $\frac{\alpha}{2}$ to the right, terminating in $G$ based on time sensing.}

We now derive this strategy by solving the preimage equations for the motion planning problem.

First, note that if the run-time executive knows that the robot is inside a particular $S_{\alpha}$, then $G$ can be reliably achieved by commanding a motion to the right. Since the robot has perfect control and time sensing, the motion can be terminated after moving 
distance $\frac{\alpha}{2}$, that is, exactly when the line $G$ is achieved. Using the preinage notation, we write this as

$$
P_{+,\left\{S_{\alpha}\right\}}(G)=\left\{S_{\alpha}\right\} \text {. }
$$

Next, we take the collection $\left\{S_{\alpha}\right\}$ as a set of subgoals, and try to find a mution that can recognizably attain this collection, and, furthermore, can distinguish which $S_{\alpha}$ the motion achieves. Consider a leftward motion starting from anywhere on the right edge $R$. The robot does not know where on $R$ the motion starts, however. To recognizably achieve some $S_{\alpha}$, such a motion should move leftward, and terminate when force-sensing indicates that $L$ has been reached. If the termination predicate measures the elapsed time of the motion, and knows the magnitude of the commanded velocity, then it can recognize which point $E_{\alpha}$ has been reached, and hence which subgoal $S_{\alpha}$ has been achieved. Writing this down in preimage equations,

$$
P_{-, R}\left(\left\{S_{\alpha}\right\}\right)=P_{-, R}\left(\left\{E_{\alpha}\right\}\right)=R .
$$

Finally, the right edge $R$ may be achieved from anywhere within the start region $T$ by moving rightward, and terminating when force sensing indicates contact. This is simply

$$
P_{+, T}(R)=T \text {. }
$$

It is instructive to examine the termination conditions for motions (1)-(3). In developing the [LMT] framework for planning guaranteed strategies, [Erdmann] developed an elegant formalization of the question, "Using sensors and history, when can the termination predicate decide that a motion has recognizably entered a goal $G_{\beta}$ ?" The answer was as follows. Assume that $G_{\beta}$ has been lifted into phase-space. Let $R$ be the start region. The forward projection, $F_{\theta}(R)$ captures the notion of history: it is all positions and velocities that can be reached given that the motion started in $R$. At a particular instant $t$ in time, let $B_{\text {ep }}(t)$ and $B_{\text {ev }}(t)$ be the sets of possible positions and velocities. These are the sensing uncertainty balls about a sensed position and velocity in phase space at time $t$. Thus sensing provides the information that the actual position and velocity must lie within the set $B_{\text {ep }}(t) \times B_{\text {ev }}(t)$. The forward projection further constrains the actual position and velocity to lie within $F_{0}(R)$. Thus the termination predicate can terminate the motion as having recognizably reached $G_{\beta}$ when

$$
F_{0}(R) \cap\left(B_{e p}(t) \times B_{e v}(t)\right) \subset G_{\beta} .
$$

Now, let $F_{0}(R, t)$ denote the time-indexed or instantaneous forward projection of $R$ under $\theta$ at time $t$. $F_{0}(R, t)$ denotes the set of positions and velocities that are possibly achievable at elapsed time $t$, under motion $\theta$, given that the motion started in $R$. The termination predicate in this case monitors a clock, in addition to position and velocity sensors. In motion (1), only the time-indexed forward projection $F_{+}\left(S_{\alpha}, t\right)$ is relevant to 
deciding termination. The motion terminates when $F_{+}\left(S_{\alpha}^{\prime}, t\right) \subseteq G$. Motion (3) can be terminated using pure force sensing. It could also be terminated using time, since there exists some $t$ for which $F_{+}(T, t)=R$. In motion $(2)$, both force sensing and time are required to terminate within a distinguishable $E_{\alpha}$. The general form of the termination condition for all three cases is as follows. The termination predicate has the form

$$
F_{\theta}(I, t) \sqcap\left(B_{\text {ep }}(t) \times B_{\text {ev }}(t)\right)\left(-G_{\beta}\right.
$$

for a goal $G_{\beta}$ and a start region $U$. (Assume that all subgoals have been lifted into phase space). In our case, position sensing error is infinite, so $B_{\text {ep }}(t)$ is $C \times J$. Let us denote $\left(C^{\prime} \times J\right) \times B_{\text {ev }}(t)$ by the simpler expression $B_{v}(t)$. Then the termination conditions for motions (1)-(3) are as follows. For the first motion (3), to terminate, we must have

$$
F_{+}(T, t) \cap B_{v}(t) \subset R .
$$

Fur the second motion (2) to terminate, we must have

$$
F_{-}(R, t) \cap B_{v}(t) \subset S_{\alpha}
$$

for some $S_{\alpha}$. We think of the termination predicate as "returning" this $S_{\alpha}$. Finally, for termination of the last motion (1), we must have

$$
F_{+}\left(S_{\alpha}\right) \cap B_{v}(t) \subset G,
$$

where the $S_{\alpha}$ in (6) is the same as the one returned by the termination predicate after the second motion as the satisfying assignment for (5).

Finally, note that time is the source of some complexity in this example. This complexity might be removed by employing a distance sensor instead. The output of such a sensor could be modeled as position sensing in $J$. The sensing action in $J$ would entail measuring the distance between $A$ and $B$. This relaxes the assumption of no position sensing in the $J$ dimensions, but such modification to the generalized configuration space framework is trivial. With this modification, $B_{\text {ep }}$ is simply regarded as a product of a position sensing ball in $C$ and a position sensing set in $J$.

This concludes the example. We have shown how to derive a multi-step guaranteed motion strategy in the presence of model error. The strategy was derived by solving the preimage equations in generalized configuration space for the motion plan. These preimage equations made the role of time- and force-sensing explicit in deriving conditions for distinguishable termination in a collection of subgoals.

\subsubsection{An EDR Strategy for Grasp-Centering}

We now generalize the grasp-center example and show how to develop an EDR strategy for this problem. 
Assume that the radius of position sensing uncertainty is larger than the diameter of $T$, but not infinite. ${ }^{12}$ Furthermore, assume that $\alpha$, the distance between $A$ and $B$, can be zero (but not negative) in the above example. That is, $D$ can be too big to grasp. Hence the hole between $A$ and $B$ can close up, as in fig. 16. Assume that the gripper starts above the height of the block $D$, in the circular region $R$ in fig. 15. Generalize the discussion of preimages above to describe an EDR strategy using preinages. We will need to consider the $y$ dimension of motion freedom as well, in the $3 \mathrm{D}$ generalized configuration space shown in fig. 16, but only the non-negative $\alpha$ in $J$. Note that EDR is "required" here, since if $\alpha$ can be zero, there exists no guaranteed strategy.

Let us rename the circular start region in fig. 15 to be $U$, and continue to use $R$ for the right edge in fig. 24. Assume that the $x-J$ slice of generalized configuration space in fig. 24 is taken at $y=0$, i.e., at the level of the table, and that under the commanded motion $v_{\theta}^{*}$, shown in fig. 15 , sliding occurs on all horizontal and vertical surfaces. However, clearly sticking will occur under $v_{j}^{*}$ on the concave left edge $L$ between $A$ and the table.

Now, let $H$ be as in fig. 19. Here is the EDR strategy in qualitative terms:

\section{Strategy EDR-Center}

E1. From $U$, command the motion $v_{0}^{*}$. Terminate on the left edge $L$ based on sticking, or in $H$ based on time.

E2. If $H$ is attained, signal failure. Otherwise, go to step (1) of strategy GuaranteeCenter.

Now, since $H_{s}=\emptyset$, the preimage equation (3a) for step (E1) simply reduces to

$$
P_{0, U}(\{L, H\})=U \text {. }
$$

At this point, the remainder of the strategy may be developed in the $x-J$ slice shown in fig. 24. To finish the preimage characterization of the EDR strategy, we must replace eq. (3), which characterizes the first step (1) of strategy Guarantee-Center, by

$$
P_{+, L}(R)=L .
$$

Note that (8) is actually a logical consequence of (3), since $L$ is a subset of $T$. Analogously, (4) must be changed by replacing $T$ by $L$. The remainder of the preimage equations (1)-(2) and (5)-(6) remain unchanged.

\footnotetext{
${ }^{12}$ This assumption is not necessary, but it simplifies our discussion somewhat.
} 


\subsection{Solving the Preimage Equations is General but Not Com- putational}

This example shows how multi-step EDR strategies under model error can be generated by solving a family of preimage equations. However, the technique is not an algorithm. We do not claim that such an algorithm could not be developed, but merely that as described above and in $|\mathrm{LMT}, \mathrm{Mason}, \mathrm{E}|$, the method is not (yet) computationally effective. ${ }^{13}$ The first reason it is non-computational is that the number of subgoals $\left\{E_{\alpha}\right\}$ and $\left\{S_{\alpha}\right\}$ is infinite. The second, and more important reason is that solving the preimage equation is, as stated, a decision problem in second-order set theory. Even if the sets are, say, algebraic, this theory is undecidable. However, there may exist a reformulation of the problem rendering it decidable. Below we describe one such reformulation, using pushforwards, which can be used in effect to solve certain "simple" preimage equations and hence to generate a restricted class of EDR plans.

\section{Push-Forwards: A Simple Generalization to $n$ - Step EDR Strategies}

The generalized preimage framework [LMT,Mason,E,D] gives a kind of "universal" inethod for generating multi-step EDR strategies. However, the technique of solving the preimage equations is not algorithmic-it is more like doing a proof by hand. For this reason, we introduce the push-forward technique for synthesizing multi-step strategies. While considerably less general than solving the full preimage equations, it leads to rather simple multi-step strategy-generation algorithms, which were implemented in LIMITED. The push-forward technique is powerful enough to generate an EDR plan for the peg-in-hole insertion strategy with model error described in section 1. However, it is not general enough to solve all steps of the grasp-centering example discussed above. This gives us a measure of the relative power of push-forward vs. preimage equation techniques.

We first introduce the "Twin Universe" example (4).

\subsubsection{The "Twin Universe" Example (4)}

Consider fig. 25. Here there are two possible universes, both in the plane, so $J$ is the two element discrete set, $\{1,2\}$. The start region is the union of $R_{1}$ in universe 1 , and $R_{2}$ in universe 2. The goal exists in universe 1 but not in universe 2. There is no one-step EDR strategy which, from the start region, can guarantee to achieve $G$ or recognize that we

\footnotetext{
${ }^{13}$ However, note that Erdmann's techniques of approximating preimages by backprojections may lead toward a fully-algorithmic method.
} 

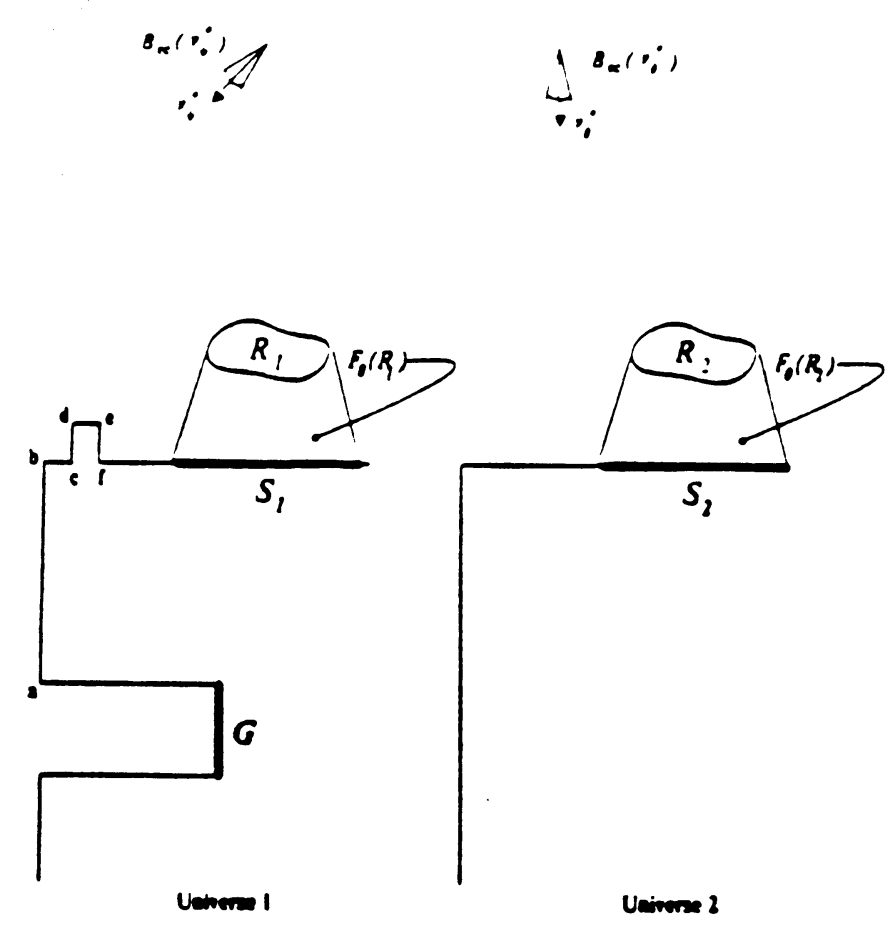

Figure 25: There are two possible universes; the goal $G$ exists in the first but not the second. The start region is $R_{1} \cup R_{2}$. Motion $\theta$ is guaranteed to move from $R_{1}$ into $S_{1}$. Motion $\psi$ is guaranteed to move from $S_{1}$ into $f$. There is an 8-step plan achieving $G$ from $R_{1}$. The forward projections of $R_{1}$ and $R_{2}$ are indistinguishable. There exists no one-step EDR strategy from the motion $\theta$.

are in universe 2. In particular, there is no one-step EDR strategy which can be derived from the motion $v_{\mathbf{0}}$.

However, there clearly exist multi-step EDR strategies. We will construct one as follows. Recall that to construct one-step EDR strategies, we took as data a goal, a start region $R$, a commanded motion $\theta$, and the preimage of the gual under $\theta$. Given this data we constructed an EDR region. From the EDR region, we attempted to construct an EDR strategy that achieved the distinguishable union of the goal or the EDR region. Now, why does this fail in fig. 25? To answer this question, let us consider what the motion $\theta$ was supposed to achieve in universe 1 . There is an 8-step plan in universe 
1 which recognizably achieves $G$ from start region $R_{1}$. It is obtained by back-chaining preimages in universe 1 . The plan moves from $R_{1}$ to the region $S_{1}$ under $v_{\theta}^{*}$. Then it slides along the top surface to vertex $f$. Next it slides to vertex $\epsilon$. It slides to the successive vertex subgoals $d$ through $a$, and then a horizontal sliding motion achieves the goal $G$.

The strategy $\theta$ is guaranteed to achieve the surface $S_{1}$ from start region $R_{1}$. Suppose we try to extend it to an EDR strategy with start region the union of $R_{1}$ and $R_{2}$. The EDR region is then simply the (cylinder over the) forward projection of the "bad" region, $F_{\theta}\left(R_{2}\right)$. (See fig. 25). There is no way that the termination predicate can distinguish between the forward projection of $R_{1}$ and the forward projection of $R_{2}$, hence no EDR strategy from $\theta$ exists.

We can easily construct a 2-step EDR strategy, however. First, we execute motion $\theta$ from the union of $R_{1}$ and $R_{2}$. This achieves a motion into $S_{1}$ in universe 1 , or into $S_{2}$ in universe 2 . The termination predicate cannot distinguish which has been attained. Suppose the second motion in the 8-step plan is $v_{\psi}^{*}$ (see fig. 25), and is guaranteed to achieve the vertex subgoal $f$ from start region $S_{1}$. We will try to construct an EDR strategy out of this second motion. Take as data: the subgoal $f$, the start region $S_{1} \cup S_{2}$, the "southwest" motion $\psi$, and the preimage of $f$ under $\psi \cdot{ }^{14}$ The EDR region for these data is the forward projection of $S_{2}$ under $\psi$ (see fig. 26). Presumably this EDR region is (eventually) distinguishable from $f$, and so we have constructed an EDR strategy at the second step. After executing the second step, we either terminate the motion as a failure, or proceed to vertex $e$, and eventually to the goal.

\subsection{Generalization: Push-Forwards}

Now, let us attempt to capture the salient aspects of the $n$-step EDR strategy construction. We take as data an $n$-step plan, with start region $R_{1}$. The actual start region is some larger region, say, $R$. Above, we had $R$ as the union of $R_{1}$ and $R_{2}$. The first motion in the plan is guaranteed to achieve some subgoal $S_{1}$ from $R_{1}$. Using this first motion from start region $R$, we try to construct an EDR region $H_{1}$, and a one-step EDR strategy that either achieves $S_{1}$ or signals failure by achieving $H_{1}$. If this succeeds, we are, of course, done.

Suppose we cannot distinguish between $H_{1}$ and $S_{1}$. In this case, we want to execute the first motion "anyway," and terminate "somewhere" in the union of $S_{1}$ and $H_{1}$. The termination predicate cannot be guaranteed to distinguish which goal has been entered.

This "somewhere" is called the push-forward of the first motion from $R$. The pushforward is a function of the commanded motion $\theta$, the actual start region $R$, the region $R_{1}$ from which $\theta$ is guaranteed, and the subgoal $S_{1}{ }^{15}$ A particular type of push-forward is defined formally in $[D]$; we describe it informally below. In example (4), the push-forward

\footnotetext{
${ }^{14}$ While $S_{1}$ is the preimage of $f$ under $\psi$ with respect to start region $S_{1}$, the preimage with respect to the entire forward projection of $S_{1} \cup S_{2}$ includes the top edge between $S_{1}$ and $f$. See secs. 2.5 and 2.4 ).

${ }^{15}$ Of course, it also depends on the termination predicate, sensing and control characteristics, etc.
} 


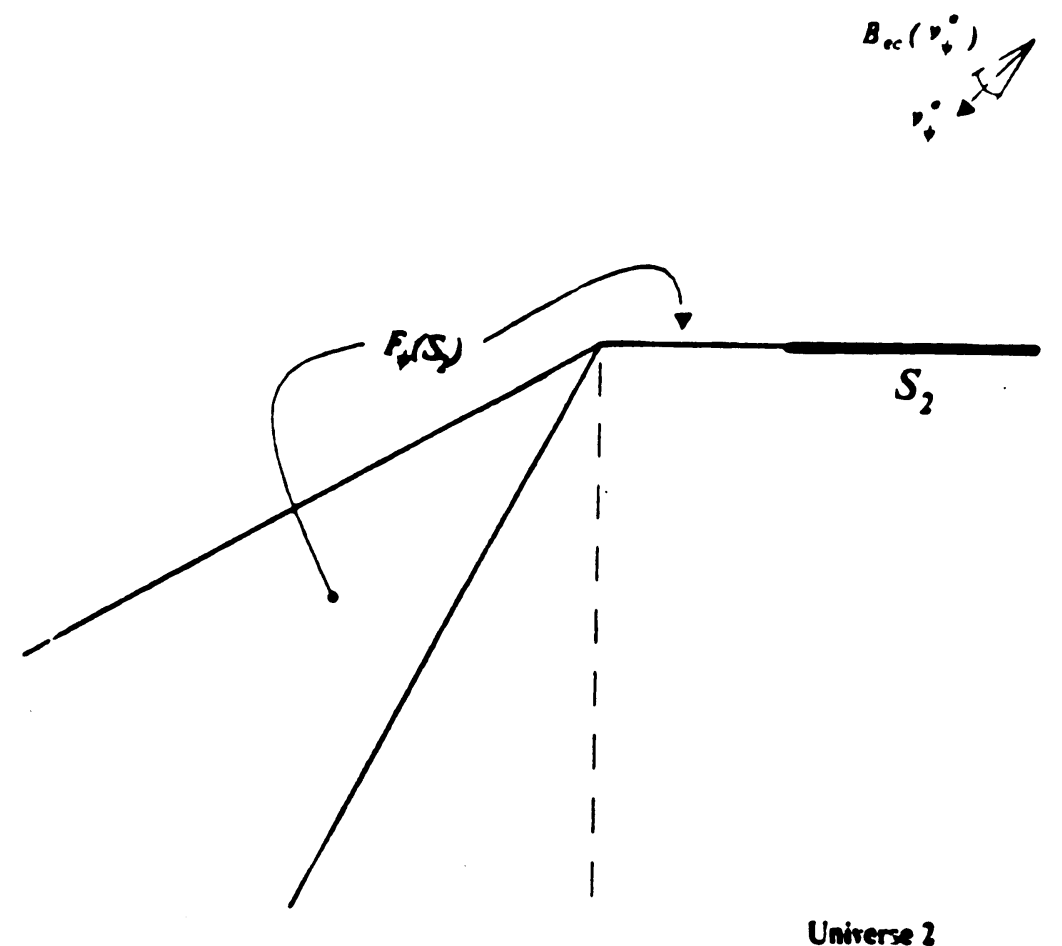

Unirerse 2

Figure 26: The forward projection under $\psi$ of $S_{2}$. 
(under $\theta$ ) of $R_{2}$ is $S_{2}$. The push-forward of $R_{1} \cup R_{2}$ is $S_{1} \cup S_{2}$. The push-forward is similar to a forward projection, except that it addresses the issue of termination. In example (4), informally speaking, the push-forward from the region $R$ (under some commanded unction $\theta$ ) is the result of executing $\theta$ from $R$ and seeing what happens. It is defined even when the strategy $\theta$ is only guaranteed from some subset $\left(R_{1}\right)$ of $R$.

Having terminated in the push-forward of $R$ (the union of $S_{1}$ and $S_{2}$ above), we next try to construct a one-step EDR strategy at the second motion of the $n$-step plan. The data are: the next subgoal $T_{1}$ after $S_{1}$ in the plan, the actual start region $S_{1} \mid 1 S_{2}$, the second commanded motion in the plan, and the preimage of $T_{1}$ under this motion. ${ }^{16}$ This defines a formal procedure for constructing $n$-step EDR strategies. At each stage we attempt to construct a one-step EDR strategy; if this fails, we push-forward and try again.

Actually, this description of the procedure is not quite complete. At each step we construct the EDR region as described. However, the one-step strategy we seek must achieve the distinguishable union of the EDR region and all unattained subgoals in the plan. That is, the EDR motion must distinguishably terminate in the EDR region, or the next subgoal, or any subsequent subgoal. This allows serendipitous skipping of steps in the plan.

By considering different data, that is, quantifying over all motions at each branch point of the $n$-step strategy, we can in principle consider all $n$-step strategies and define non-directional EDR strategies. This is at least as difficult as computing $n$-step nondirectional preimages. If we wish to consider plans of different lengths, we must also quantify over all $n$. Needless to say, the branching factor in the back-chaining search would be quite large.

\subsection{More on the Push-Forward}

The problem of defining the push-forward may be stated informally as follows: "Where should the motion be terminated so that later, after some additional number of pushforwards, a one-step EDR strategy may be executed."

Many different push-forwards can be defined. Using the notation above, note the motion is not even guaranteed to terminate when executed from $R$ : it is only guaranteed from $R_{1}$. This means that velocity-thresholding and time may be necessary in the termination predicate. There are other difficulties: for example, a priori it is not even necessary that entry into the union of the subgoal $S_{1}$ and the EDR region $H_{1}$ be recognizable. Thus defining the push-forward is equivalent to defining where in $S_{1} \cup H_{1}$ the motion can and should be terminated.

Depending on that push-forward is employed, we may or may not obtain an $n$-step EDR strategy. It is possible to define constraints on the push-forward that must be satisfied to ensure that a strategy will be found if one exists. These constraints are given

\footnotetext{
${ }^{16}$ The preimage is with respect to the forward projection of the actual start region $S_{1} \cup S_{2}$.
} 
in |D|. While in the appendix we can give equations that the push-forward must satisfy, at this time a constructive definition is not known. This situation is similar to, and possibly harder than the problem of solving the general pre-image equation.

\subsection{An Approximation to the Push-Forward}

We may have to approximate the desired push-forward. We give such an approximation here. We provide it to show what the push-forwards alluded to above are like. Such approximate push-forwards may prove useful in approximating the desired push-forward. The issue deserves more study. Since this approximate push-forward is incomplete, the reader should consider its description here as illustrative of the research problem, and uot as an endorsement.

The push-forward employed in example (4) was formed by "executing the strategy anyway, and seeing where it terminated." How do we formalize this idea? Consider the termination predicate as a function of the starting region, the initial sensed position, the commanded velocity, the goal(s), and the sensor values. The sensor values are changing; the predicate monitors them to determine when the goal has been reached. Now, if the termination predicate "knew" that in example (4) the start region was the union of $R_{1}$ and $R_{2}$, then the first motion strategy $\theta$ could never be terminated: the predicate could never ensure that the subgoal $S_{1}$ had been reached. This is simply because $S_{1}$ and $S_{2}$ are indistinguishable. But if we "lie" to the termination predicate and tell it that the motion really started in $R_{1}$, then the predicate will happily terminate the motion in $S_{1} \cup S_{2}$, thinking that $S_{1}$ has been achieved. Viewing the termination predicate as a function, this reduces to calling it with the "wrong" arguments, that is, applying it to $R_{1}$ instead of $R_{1} \cup R_{2}$. The push-forward we obtain is "where the termination predicate will halt the motion from all of $R_{1} \cup R_{2}$, thinking that the motion originated in $R_{1}$." $S_{2}$ is obtained as the set of places outside of $S_{1}$ where the lied-to termination predicate can halt.

Even formalizing the construction of this simple push-forward is subtle; details are given in [D]. While this approximate push-forward is incomplete, it does suffice for a wide variety of EDR tasks. The approximate push-forward captures the intuitive notion of "trying the strategy anyway, even if we're not guaranteed to be in the right initial region." It is incomplete because it fails to exploit sufficiently the geometry of the forward projection of the "bad" region. Better push-forwards must be found; this one is merely illustrative of the problems.

\subsection{Notation for Push-Forwards}

For a motion $\theta$ from start region $R$, we denote the general push-forward by $F_{* \theta}(R)$. That is, $F_{* 0}(R)$ is that subset of the forward projection $F_{\theta}(R)$ where the motion $\theta$ will terminate when executed from $R$. 
LIMITED employs an approximate push-forward. It is called the a priori push-forward based on sticking termination, and is defined as that subset of the forward projection where sticking is possible. This push-forward is correct as long as all motions use sticking as a termination condition. For a set $U$ we define push ${ }_{\theta}(U)$ as all points in $U$ where sticking is possible. Where possible, we will develop the EDR theory for the general push-forward which employs the full power of the termination predicate. In places, however, and when describing LIMITED, we will specialize the push-forward to use sticking termination alone, and thereby set $F_{* \theta}(R)=\operatorname{push}_{\theta}\left(F_{\theta}(R)\right)$.

\subsection{Sticking as a Robust Subtheory of EDR}

In the abstract EDR theory, one envisions the run-time termination predicate performing whatever computations are necessary to terminate a motion recognizably in $G$ or $H$. That is, in principle, the planner decides what termination conditions are appropriate for a successful EDR strategy, and encodes them into the motion strategy. Of course, it is also the responsibility of the planner to verify that this encoding will always result in a distinguishable termination. In short, the abstract EDR theory can employ the full power of the [LMT] preimage framework to generate motion strategies.

However, LIMITED employs only certain restricted termination conditions, as we saw above. In particular, sticking is used in most experiments. This restricts the class of strategies Limited can generate. The restriction requires some justification, and that is the purpose of this section.

First, recall that in polyhedral environments with a bounding box, sticking termination is sufficient to ensure that all pure translations eventually terminate [Buckley|. In general, in this work we have made the heuristic assumption that motions can eventually be terminated via sticking. Failing this, we also entertain the weaker assumption that if sticking is insufficient, then time can be employed to wait until $G \cup H$ has been achieved before termination. ${ }^{17}$

To analyze the structure of sticking termination, let us introduce the following notation. If the robot recognizably achieves $G \cup H$, this means that the run-time executor can determine that $G$ or $H$ has been achieved, but cannot necessarily tell which of $G$ or $H$ has been entered. If the robot recognizably achieves $\{G, H\}$, then it can further distinguish which of $G$ or $H$ it has reached. $G \cup H$ is called the union while the set notation is called the distinguishable union.

Throughout this section we assurne without loss of generality that the goal $G$ is contained within the forward projection ${ }^{18}$ If this is not the case, then intersect them to obtain a new goal.

Limited tries to decompose this problem-of ensuring that all trajectories terminate recognizably in $\{G, H\}$-into two subproblems. The first is to ensure that the motion

${ }^{17}$ See sec. 8.3 of [Donald, 87].

${ }^{18}$ This is not a severe restriction; see sec. 7.3 of [Donald, 87]. 
in fact terminates in $G \cup j$. That is, the problem is to determine that at least one of (; or $H$ has been achieved, although the robot may not know which. The second problem is to distinguish between $G$ and $H$, once $G \cup j H$ has been achieved.

Note that the first problem requires distinguishing between $G_{1}, H$ and its complement. Here is the key point:

- The construction of $H$ guarantees tautologously that with sticking termination, $G \cup H$ will be recognizably achieved when the motion terminates. That is, with sticking termination, no motion can terminate outside of $G \cup H$.

This resolves the first subproblem. Thus

With sticking termination, all candidate one-step EDR strategies eventually terminate recognizably in $G \cup H$ (but not necessarily in $\{G, H\}$ ). Of these, all valid $E D R$ strategies can distinguish between $G$ and $H$ after termination, and hence recognizably terminate in $\{G, H\}$.

The second subproblem is how to distinguish between $G$ and $H$ once $G \cup H$ has been achieved. Ising sensors and history, the termination predicate can decide that a motion has recognizably entered a (phase-space) goal $G_{\beta}$ when

$$
F_{\theta}(R) \cap\left(B_{\text {ep }}(t) \times B_{\text {ev }}(t)\right) \tau_{-} G_{\beta} .
$$

(See sec. 4.1). Now, when is it the case that the termination predicate can distinguish which of $G$ or $H$ has been reached? Exactly when $(*)$ is true for $G_{\beta}$ in $\{G, H\}$. However, in our case, sticking termination guarantees that the actual position and velocity lie within $G \cup H$. Furthermore, $G \cup H$ is a subset of the forward projection, and $G$ and $H$ are disjoint by construction. The forward projection provided no further constraint in distinguishing between $G$ and $H$. Thus history plays no role in the run-time distinguishing actions of the robot executive; history has been pre-encoded into the structure of $H$. Hence, we can predict that the run-time executor can distinguish which of $(r$ or $H$ has been achieved when the planner can predict that $G$ and $H$ are distinguishable using sensors alone. A procedure-albeit not completely general-for deciding this question was described in 2.4 .

\subsubsection{Generalizations}

There are several possible generalizations of these termination techniques. First, it may be possible for the run-time executor to use time to ensure that the motion terminates in $G \cup H$. That is, forward projections may, in principle, be indexed by time. Hence in $(*), F_{\theta}(R)$ is replaced by $F_{\theta}(R, t)$, the instantaneous forward projection of $R$ under $\theta$ at time $t$, which is typically much smaller. See sec. 4.1. The termination predicate in this case monitors a clock, in addition to position and velocity sensors. However, in this 
case, history (by which we mean $F_{\theta}(R, t)$ ) could be employed to distinguish $G$ from $H$, even though the motion had terminated recognizably in $G \cup H$. The reason for this is that the time-indexed forward projection has not been pre-encoded into the structure of $H$. That is, $H$ was constructed using the timeless forward projection, which the union of all time-indexed forward projections. Hence, we can summarize these observations as follows:

- If a termination predicate without time uses sticking to terminate the motion, then distinguishing $G$ from $H$ is a history-free decision. However, for a termination predicate with a sense of time, the decision is not history-free.

Thus sticking subtheory does not preclude more general termination techniques based on position, force, and time sensing. However, two computational issues become more difficult. First, sticking termination is a robust method for ensuring termination in $G \cup H$. With time termination, or more general position/force termination criteria, it is more difficult to ensure termination in $G \| H$-although admittedly these criteria are more powerful. Second, after sticking termination, deciding between $G$ and $H$ is historyfree. With more general termination predicates, history can provide extra constraint in distinguishing between $G$ and $H$.

Finally, note that [Buckley] recognized the value of sticking termination when implementing an [LMT] planner for guaranteed strategies in $\Re^{3}$. His planner used sticking termination. In particular, he provided certain criteria for guaranteeing that a strategy eventually terminates in sticking. Buckley's criteria amount to ensuring that the environment is finite polyhedral, within a bounding polyhedral box.

\subsection{Example: Multi-step EDR Plan for Peg-in-Hole with Model Error}

The advantage of the push-forward technique is that it can be made computational. We now give LIMITED's algorithm for generating multi-step strategies using push-forwards, and describe an experiment which used this method.

Recall section 1, figs. 6-16, which described a two-step EDR plan for a peg-in-hole plan with 3 DOF model error. Here is how this multi-step strategy was generated:

\section{Algorithm Multi}

1. First, try to generate a one-step EDR strategy using the algorithm IEDR in sec. 2.4.

Suppose this fails. Then: 
2. Generate a commanded velocity $v_{\theta}^{*}$, such that the forward projection of the start region intersects the goal in some slice.

3. Compute the EDR region $H$ for $v_{\theta}^{*}$.

4. Compute the sticking push-forward of the motion, $R_{1}=\operatorname{push}_{\theta}(G|| H)$.

5. Using $R_{1}$ as the start region, generate a one-step EDR strategy using algorithm IEDR.

Of course, in LIMITED the computation is memoized so that the projection and EDR regions computed in step (1) are not recalculated in steps (2) and (3). Obviously, we can extend this algorithm to generate longer strategies which push-forward several times and finally terminate in a single-step EDR strategy.

Now, LIMITED is a multi-resolution planner. The algorithm outlined above generates a multi-step strategy at a single resolution. The resolution of planning is simply the set of $\alpha$ values in which slices are taken. A resolution $S_{1}$ is finer than $S_{2}$ if it contains more slices. The multi-resolution outer loop works like this:

M1. At a coarse resolution, generate a multi-step EDR strategy $\theta_{1}, \ldots, \theta_{n}$ using the forward-chaining single-resolution algorithm above.

M2. Select a finer resolution. Use the directions $\theta_{1}, \ldots, \theta_{n}$ as a suggested strategy and attempt to verify that it is an EDR strategy at the finer resolution.

M3. If $\theta_{1}, \ldots, \theta_{n}$ is not an EDR strategy at the finer resolution, try to modify it so that it is, by using $\theta_{1}, \ldots, \theta_{n}$ as suggested directions, and searching nearby directions at all levels.

The process terminates when the resolution is finer than some predetermined level. ${ }^{19}$ The critical slice method described in [Donald, 87] may be one way to obtain such an a priori bound and know that it is sufficient. In LIMITED, however, the bound is a user input because otherwise the number of slices required would be prohibitive.

In the peg-in-hole example there were 3DOF of model error: the width of the hole, the depth of the chamfers, and the orientation of the hole. The resolutions used in planning the two-step strategy were as follows:

R1. Holding orientation fixed, 4 slices of the depth $\times$ width axes.

R2. Holding orientation fixed, 16 slices of the depth $\times$ width axes.

\footnotetext{
${ }^{19} \mathrm{Or}$, when at some level, no EDR strategy can be found.
} 
R3. Holding orientation fixed, 72 slices of the depth $\times$ width axes.

R4. 100 slices of the depth $\times$ width $\times$ orientation axes.

For detailed output traces of Limited, please see [Dunald, $8 T$ ].

\subsection{The Loss of Power with Push-Forward Techniques}

While push-forwards permit us to develop simple algorithms for generating multi-step strategies, clearly these algorithms are theoretically less powerful than solving the preimage equations in full generality. We now attempt to give an intuitive characterization of the loss of power. In particular, push-forwards are general enough for the peg-in-hole EDR strategy with model error. However, they are not general enough to generate the grasp-centering plan. We now discuss where in the grasp-centering example the pushforward techniques are inadequate. The key point is this: if each commanded motion and termination condition could be non-deterministically "guessed," and a push-forward for each motion and termination condition could be computed, then in the grasp-center example this would suffice to generate a strategy. However, the push-forward algorithms we have developed are not powerful enough to do this.

First, let us derive the push-forwards of each motion in strategies EDR-Center and Guarantee-Center. Recall that $E l$ is the first step of the EDR plan, and motions 1,2 , and 3 are steps in the (subsequent) guaranteed plan. In the third column we note whether or not the push-forward technique is computationally effective for this motion.

\begin{tabular}{cccc} 
Motion & Push - Forward & & Computational? \\
\cline { 3 - 4 } & & & \\
1 & $L, H\}$ & & yes \\
2 & $R$ & yes \\
3 & $\left\{E_{\alpha}\right\}$ & no \\
$G$ & $G$ & no
\end{tabular}

The push-forwards for motions $E 1$ and 1 can be computed using the algorithms of [Donald, 87) (see sec. 2.4) and algorithm Multi above. In motion $E 1, L$ may be found using sticking termination. $H$ may be found using time, or position and force sensing termination. In motion $1, R$ may be found using contact, or sticking termination. However, our algorithms cannot compute the push-forward $\left\{E_{\alpha}\right\}$, which contains an infinite number of components. Furthermore, we have not developed algorithms for computing push-forwards based on time-termination (except for elapsed time termination, of the form "terminate anytime after $t$ seconds"). Thus the push-forward $G$ for the last motion cannot be computed by our algorithms either. 


\subsubsection{Discussion}

Let us pause to review. We first described a fully-general, but non-computational technique for generating multi-step strategies. This method-solving the preimage equations-was applied to the grasp-centering example. Next the push-forward techniques were introduced as a computational, although less powerful approach to the synthesis of multi-step strategies. Push-forward algorithms were described, and we saw how LIMITED used these techniques to generate a two-step plan for the peg-in-hole problem with model error. Finally, we discussed the limitations of the push-forward techniques. We saw that they were not powerful enough to solve the grasp-center problem in its entirety. By describing an experiment where push-forwards sulfice, and showing an example where they are insufficiently general, we have tried to give an intuitive but fairly precise characterization for the relative power of push-forwards.

\section{Failure Mode Analysis}

Push-forward techniques require a precise geometrical characterization of the forwardprojection, and algorithms for computing it. The gear-meshing example of sec. 1 is a problem in a four-dimensional generalized configuration space with pushing. Two of the dimensions are rotational: one of these can be commanded, and the other cannot, but the position along this dimension may be changed via pushing. It is difficult to develop good forward projection algorithms in this generalized configuration space, although criticalslice methods are a start. For this reason, a different technique was developed for planning multi-step strategies in this domain. It is applicable for any generalized configuration space with the same degrees of freedom and pushing characteristics (that is, any polygonal shapes in place of the gears). The new technique is called failure mode analysis; we describe it in this section.

Failure-mode analysis is a method for synthesizing multi-step strategies using a kind of "approximate" or "a priori" forward projection. At first glance, it may appear unrelated to push-forward or preimage techniques. However, in the next section, on the weak EDR theory, we present a viewpoint which essentially "unifies" the three approaches.

\subsection{Introduction to Failure Mode Analysis}

Recall the gear-meshing plan LIMITED generated in sec. 1, fig. 4. In particular, we suppose that vision is poor, or that the gears are accessible to the robot gripper, but not to the camera. This means that position sensing will be very inaccurate, and hence may be of no use to determine whether the gears are successfully meshed. This will often be the case in practice. In this case, force sensing must be used to disambiguate the success of the first motion (meshing) from failure (jamming in an unmeshed state). A multi-step strategy is required. While pushing of $B$ can change its orientation, and hence cause 
motion across $J$ (sec. 2.2.2) our focus here will be on the multi-step strategy generation and not on the physics of pushing.

In the gear-meshing plan, motion $\theta_{2}$ is used to disambiguate the result of motion $\theta_{1}$. The technique used is failure mode analysis. LIMITED is given a repertory of qualitative failure modes, which comprise sticking and breaking contact. Motion $\theta_{1}$ can end in a "good" region (meshed) or a "bad" region (jam). Limited tried to generate a disambiguating motion as a second step. This motion is required to terminate in a failure mode fromi all "bad" regions.

Here is how Limited generates motion $\theta_{2}$. Let $H$ be the EDR region for motion $\theta_{1}$. The planner determines all configurations where motion $\theta_{1}$ can terminate outside of $G$. Call this region push $\theta_{\theta_{1}}(H)$. push $_{\theta_{1}}(H)$ then forms the start region for motion $\theta_{2}$. Limited then uses quasi-static analysis to "prove" that when $A$ is at any configuration in push $\theta_{\theta_{1}}(H)$, and a pure rotation of $A$ is commanded, that all possible motions of $A$ result in sticking or breaking contact. Sticking and breaking contact are called failure modes; there is a class of EDR plans which can be terminated in failure when sticking or breaking contact are detected. EDR planning with failure modes constitutes a robust subtheory of EDR. It is a subtheory because assuming this kind of failure mode is a restrictive assumption to make planning tractable. It is robust because sticking and breaking contact are easy to recognize, relatively speaking, as failure modes by a run-time robot executor.

From the preimage point of view, failure modes are implemented simply as different classes of termination predicates.

\subsection{Specifying the Goal: Functional Descriptions}

Recall our discussion of sticking as a termination condition in chapter Sticking had the advantage of ensuring "good" behavior in the EDR region $H$. In particular, it could be guaranteed that all motions would eventually terminate in $G \cup H$, rendering the distinguishability of $G$ vs. $H$ a history-free decision. However, in order for a sticking termination predicate to generate good EDR plans, it was in fact necessary to ensure that the motion strategy has "good" behavior at the goal as well. In particular, the commanded motion should stick at the goal.

In failure mode analysis, we have a similar situation. The purpose of motion $\theta_{2}$ is to force all motions starting from push $_{\theta_{1}}(H)$ to terminate in sticking or breaking contact. Clearly this is only useful if no motion from push $\theta_{\theta_{1}}(G)$ can even possibly terminate in sticking or breaking contact. This is the required "good" behavior at the goal. Thus, in an EDR plan generated by failure mode analysis,

F1. Under motion $\theta_{2}$, all motions starting from push $_{\theta_{1}}(H)$ must terminate in a failure mode.

F2. No motion frorn push $_{\theta_{1}}(G)$ can possibly terminate in a failure mode. 
F3. The goal is a fixed-point under motion $\theta_{2}$.

Limited decides whether or not $(\mathrm{F} 1)$ is true. However, $(\mathrm{F} 2)$ is given as input to Limited. We will now discuss how $(F 2)$ is specified. In the next section we will describe algorithms for computing (F1). (F3) may be decided using forward projections; the actual condition we require is

$$
F_{\theta_{2}}\left(\operatorname{push}_{\theta_{1}}(G)\right) \subset G
$$

which is implied by the fixed-point equation

$$
F_{\theta_{2}}(G)=G,
$$

since of course $\operatorname{push}_{\theta_{1}}(G)$ is contained in $G$.

The goal state for gear meshing may be viewed purely geometrically. That is, it may be viewed as a set in generalized configuration space. This view is useful for computing the EDR regions. Alternatively, the goal may be specified through a functional description. For example, we might specify the goal as a difference equation (DE). The intuition behind this difference equation formulation of the goal is, "In the goal, any finite rotation of A results in an equal and opposite rotation of $B$." More precisely, the difference equation specifies:

DE. Command any non-zero finite rotation $\Delta \alpha_{1}$ to A. In the goal, this results in a finite rotation of $A$ by $\Delta \alpha_{1}$ and of $B$ by $-\Delta \alpha_{1} \cdot{ }^{20}$

This difference equation captures the functional aspects of the gears in their meshed state. Now, it is clear that this equation may be "differentialized." That is, we consider it to be true for all non-zero displacements, no matter how small. If this is the case, then it is clear that breaking contact is in direct contradiction to the truth of the difference equation (DE). This is because if contact is broken, then there exists some finite rotation of $A$ that will not affect the orientation of $B$. Similarly, sticking contradicts the truth of the difference equation, for if the gears stick, then they are not properly meshed, i.e., we do not obtain equal and opposite rotations.

In LIMITED failure mode analysis, we view the goal state as a combined geometrical and functional specification. In [Donald, 87), we considered three ways of specifying the functional aspects of the goal. The last, which decides questions about goal predicates via the theory of real closed fields, is only of theoretical interest. The second is a heuristic approximation to such an inference engine. The first is a more robust solution with an engineering flavor. It places on the user the burden of ensuring well-behaved qualitative behavior at the goal. We will confine ourselves to the first, and simplest method in this paper:

\footnotetext{
${ }^{20} . A$ and $B$ are the same size. Clearly, this may be generalized to different pitch gears.
} 


\subsubsection{Specifying the Functional Aspects of the Goal}

Method 1. L'ser input. In this method, it is the responsibility of the user to ensure that $(\mathrm{F} 2)$ is true. That is, the user must guarantee that failure modes cannot occur at the goal. This, of course, is the easiest method. If the user guarantees that (F2) holds, then it remains only for Limited to show (Fl).

Some of the greatest and most interesting unsolved problems in geometrical robotics lie in the interaction of functional and geometrical descriptions of goals. In particular, we would like to devise algorithms for computing a geometrical goal region given a functional description-for example, a quantified difference equation-for the desired behavior in the goal state. Conversely, we would like to be able to infer a functional description of the goal from its geometrical aspects. The latter would be useful in automatically generating termination predicates to recognize the goal.

\subsection{Approximate Algorithms for Failure Mode Analysis}

We now describe algorithms for deciding whether

F1. Under motion $\theta_{2}$, all motions starting from push $_{\theta_{1}}(H)$ must terminate in a failure mode.

Let us denote push $_{\theta_{1}}(H)$ by $H_{1}$. These algorithms use time-indexed forward projections to prove that under $\boldsymbol{\theta}_{2}$, all paths starting in $H_{1}$ eventually stick or break contact. The algorithms are approximate, although conservative. That is, if they terminate then $(F 1)$ is true. However, they may not terminate if (F1) is false, and they may miss cases where $(\mathrm{Fl})$ is true. The accuracy of the algorithm increases as the time steps for the time-indexed forward projections are taken to be finer. In the $4 \mathrm{D}$ generalized configuration space for the gears, which is $\Re^{2} \times S^{1} \times S^{1}$, these time-steps correspond to the fineness of the slice resolution across the rotational dimensions.

We will first describe a quite general algorithm for deciding (F1). It is applicable wherever we can obtain a computational characterization of time-indexed forward projections. Later, we will give a specialized algorithm in the generalized configuration space for the gears, and show that it is in fact a special case of the general algorithm.

\subsubsection{A General Algorithm}

The basic idea is to step along in time, simulating the motion, and determine whether or not it breaks contact or sticks. Of course, we must simulate all possible motions, using forward projections. 
First we must develop some notation. Recall that for a planar set $H_{1}, \partial H_{1}$ denotes its obstacle edges. Here, we will use it to more generally to denote the obstacle surfaces (as opposed to the free-space surfaces) bounding a set $H_{1}$ in generalized configuration space. (In our case $H_{1}$, the input to the algorithm, is the push-forward of motion $\theta_{1}$ ).

Let $x$ be a point in generalized configuration space. Then stick $k_{\theta}(x)$ is true if sticking is necessary at $x$ under all control velocities $B_{e c}\left(v_{\theta}^{*}\right)$ consistent with the nominal commanded velocity $v_{\theta}^{*}$. Let stick $H_{\theta}\left(H_{1}\right)$ denote all points $x$ in $H_{1}$ where stick: $(x)$ holds.

Now, assume some positive minimum modulus bound on the commanded velocity. We use $f_{\theta, \Delta_{\ell}}(\cdot)$ as the time-indexed forward projection operator (see $[$ Erdmann]). So $F_{\theta, \Delta t}\left(H_{1}\right)$ denotes the set of possible positions the robot can be at at time $\Delta t$, having started in $H_{1}$ at time $t=0$.

Now, we are ready to give the general algorithm for deciding $(\mathrm{F} 1)$ :

\section{Algorithm Gen}

1. Let $F-F_{\theta, \Delta t}\left(H_{1}\right)$.

2. Let $H_{2}-\partial F-$ stick $_{\theta_{2}}(\partial F)$.

3. When $H_{2}=\emptyset$, we have proven that all paths from $H_{1}$ must eventually stick or break conlact. Halt.

4. Else, $H_{1}-H_{2}$. Goto (1).

Note that $H_{1}$ is permitted to be in free-space, although given the sticking push-forward it will, in fact, always be on a generalized configuration space boundary. Note that Gen is a semi-decision procedure. Clearly, if the algorithm halts, then all paths originating in $H_{1}$ eventually break contact or stick. Fig. 27 illustrates the algorithm. Suppose the $H_{1}$ region is the edge $e$. Its forward projection after $\Delta t$ is the region $U \cup g$. The obstacle edges of the forward projection are $e^{\prime}, f$, and $g$. Sticking must occur on $f$. Hence, $H_{2}$ is $e^{\prime} \cup g$.

We now mention a basic property of forward projections that this algorithm exploits. It is the property that forward projection commutes with union. In particular, if we have

$$
H_{1}=\overbrace{H_{B}}^{\text {boundary }}+\overbrace{H_{F}}^{\text {free-space }} .
$$

then

$$
F_{\theta}\left(H_{1}\right)=F_{\theta}\left(H_{B} \cup H_{F}\right)=F_{\theta}\left(H_{B}\right) \cup F_{\theta}\left(H_{F}\right) .
$$





Figure 27: Wustration of the general algorithm. The start region $H_{1}$ is the edge e. Its forward projection after $\Delta t$ is the region $U \cup g$. The obstacle edges of the forward projection are $e^{\prime}, f$, and $g$. Sticking must occur on $f$. Hence, $H_{2}$ is $e^{\prime} \cup g$. 
This key property permits the algorithm to decompose the failure mode analysis into essentially independent decision problems about the forward projections of the free-space, sliding, and sticking regions in the push-forward.

\subsubsection{A Specialized Algorithm}

For failure-mode analysis, Limited employs an algorithm that is a special case of the general algorithm above. The idea is that when commanding a pure rotation of $A$, the time-indexed forward projection across slices can be well approximated by the differential forward projection. The differential forward projection is a technique for propagating the forward projection across slices, when rotations of $A$ and $B$ are permitted. Recall our notation for motions $\theta_{1}$ and $\theta_{2}$. $\theta_{1}$ is a commanded pure translation of $A$, and may be viewed as unit vector $v_{\theta_{1}}^{*}$ in the plane. $\theta_{2}$ is a commanded pure rotation of $A$, and may be viewed as a member of $\left\{+d \alpha_{1},-d \alpha_{1}\right\}$, for positive and negative commanded rotations.

\section{Differential and Propagated Forward Projections}

Pure Translations. Forward projections must be propagated between slices even when a pure translation is commanded, since a pure translation $\theta_{1}$ can alter the orientation of $B$, and hence the slice-value, through pushing. Here is how the differential forward projection is constructed for a pure translation $\theta_{1}$. Let $\left(x, y, \alpha_{1}, \alpha_{2}\right)$ denote a configuration in the generalized configuration space for the gears, $\Re^{2} \times S^{1} \times S^{1}$. $\left(x, y, \alpha_{1}\right)$ denotes a configuration of $A . \alpha_{2}$ denotes the configuration of $B$. Hence, we regard the orientation of $B$ (the "last" $S^{1}$ in the product) as $J$. Now, $H_{1}$ is a set in generalized configuration space. Let $\left.H_{1}\right|_{\alpha_{1}, \alpha_{2}}$ denote a particular $x$-y slice of $H_{1}$ for orientation $\alpha_{1}$ of $A$ and $\alpha_{2}$ of B.

Motion $\theta_{1}$ commands a pure translation of $A$. Now, for each edge in $\left.H_{1}\right|_{a_{1}, \alpha_{2}}$, LIMITED performs a quasi-static analysis to determine the possible impending motions of $A$ and $B$. That is, it determines which way(s) $A$ and $B$ can rotate. These directions may be viewed as tangent vectors to the pure rotational dimensions of generalized configuration space. The set of possible directions may be identified with a set of pairs

$$
\left\{-d \alpha_{1}, 0,+d \alpha_{1}\right\} \times\left\{-d \alpha_{2}, 0,+d \alpha_{2}\right\}
$$

in the tangent space to $\left(S^{1} \times S^{1}\right)$. By performing this analysis for all edges, we obtain a set of directions,

$$
d F_{\boldsymbol{\theta}_{1}}\left(\left.H_{1}\right|_{\boldsymbol{\alpha}_{1}, \boldsymbol{\alpha}_{2}}\right) \text {, }
$$

which is called the differential forward projection of $\left.H_{1}\right|_{\alpha_{1}, \alpha_{2}}$ under $\theta_{1}$. It is assumed that commanding $\theta_{1}$ from region $\left.H_{1}\right|_{\alpha_{1}, \alpha_{2}}$ can result in any motion direction in this set. 
Suppose $\left(\alpha_{1}^{\prime}, \alpha_{2}^{\prime}\right)$ is a slice taken in the direction of some tangent vector $\mathbf{v}$ in the differential forward projection. For example, if $\mathbf{v}=\left(+d \alpha_{1},-d \alpha_{2}\right)$, then $\alpha_{1}^{\prime}=\alpha_{1}+\epsilon_{1}$ and $\alpha_{2}^{\prime}=\alpha_{2}-\epsilon_{2}$ for some small positive scalars $\epsilon_{1}$ and $\epsilon_{2}$.

Now, the forward projection may be propagated to the adjacent slice $\left(\alpha_{1}^{\prime}, \alpha_{2}^{\prime}\right)$ as follows. An edge $e_{i}$ in $\left.H_{1}\right|_{\alpha_{1}, \alpha_{2}}$ corresponds to the intersection of an algebraic surface $V$ in generalized configuration space with the "plane" $\Re^{2} \times\left\{\left(\alpha_{1}, \alpha_{2}\right)\right\} . V$ is followed into $\left(\alpha_{1}^{\prime}, \alpha_{2}^{\prime}\right)$, and the forward projection of $e_{i}$ is taken to be the intersection of $V$ with the "plane" $\Re^{2} \times\left\{\left(\alpha_{1}^{\prime}, \alpha_{2}^{\prime}\right)\right\}$. In this manner, we obtain a set of edges $\left\{\epsilon_{2}^{\prime}\right\}$ in the new slice. The pure translational forward projection of these edges under $\theta_{1}$ is then computed within this slice, so the propagated forward projection is $F_{\theta_{1}}\left(\left\{e_{i}^{\prime}\right\}\right)$. This propagated forward projection is computed at a fixed orientation of $A$ and $B$. Ideally, the planner should decide whether the sliding characteristics change along $V$ while moving through rotation space. The rotational values which are sliding-critical are discussed in 6.3 of [Donald, 87]. ${ }^{21}$ The propagated forward projection increases in accuracy as the slices are taken closer together.

Pure Rotations. Consider the problem of computing forward projections across slices for a commanded pure rotation $\theta_{2} \in\left\{+d \alpha_{1},-d \alpha_{1}\right\}$. For simplicity, we first consider the case where $H_{1}$ consists of a single point. Let $\mathbf{x}$ be a point in the plane, and $\left(\mathbf{x}, \alpha_{1}, \alpha_{2}\right)$ be a configuration where $A$ and $B$ are in contact. Then the differential forward projection of $x$ under $\theta_{2}$ will consist of vectors in the set of eq. (9). The differential forward projection has the same structure as in the pure translational case. It may be computed using quasi-static analysis. (see the next subsection below).

Suppose for the sake of development that the differential forward projection consists of exuctly one direction $\mathbf{v}$, and that $\left(\boldsymbol{\alpha}_{1}^{\prime}, \boldsymbol{\alpha}_{2}^{\prime}\right)$ is an adjacent slice in that direction, as above. Now we ask, what is the propagated forward projection of $x$ into the adjacent slice, $\left(\alpha_{1}^{\prime}, \alpha_{2}^{\prime}\right)$ ? Well, it can be one of two things: either it is $\mathbf{x}$, or it is empty. The reason is that $x-y$ position is invariant ${ }^{22}$ under $\theta_{2}$. Thus, an upper bound on the propagated forward projection of $\left.H_{1}\right|_{\alpha_{1}, \alpha_{2}}$ into an adjacent slice $\left(\alpha_{1}^{\prime}, \alpha_{2}^{\prime}\right)$ is found by simply "copying" 23 $\left.H_{1}\right|_{\alpha_{1}, \alpha_{2}}$ into slice $\left(\alpha_{1}^{\prime}, \alpha_{2}^{\prime}\right)$.

Now, consider the propagated forward projection of $\left(\mathbf{x}, \alpha_{1}, \alpha_{2}\right)$, under motion $\theta_{2}$, into slice $\alpha_{1}^{\prime}, \alpha_{2}^{\prime}$. It is simply the point $\left(\mathbf{x}, \alpha_{1}^{\prime}, \alpha_{2}^{\prime}\right)$. There are three possible qualitative outcomes:

1. $x$ is inside a generalized configuration space obstacle in slice $\left(\alpha_{1}^{\prime}, \alpha_{2}^{\prime}\right)$.

2. $x$ is in free space in slice $\left(\alpha_{1}^{\prime}, \alpha_{2}^{\prime}\right)$.

\footnotetext{
${ }^{21}$ Detecting sliding critical orientation parameters along the algebraic surface $V$ has not been impleinented in LIMITED. Thus the propagated forward projection may be larger than it need be.

${ }^{22}$ See below for more on this assumption.

${ }^{23}$ We use the awkward term "copying" instead of "translating", since while the latter is precise mathematically it is confusing robotically.
} 
3. $\mathbf{x}$ is on the boundary of a generalized configuration space obstacle in slice $\left(\alpha_{1}^{\prime}, \alpha_{2}^{\prime}\right)$.

Obviously, (2) implies that contact has been broken. (1) corresponds to a physically impossible situation. Since the configuration $\left(\mathbf{x}, \alpha_{1}^{\prime}, c x_{2}^{\prime}\right)$ is physically unattainable, this means that the commanded motion $\theta_{2}$ must result in sticking (no actual motion) before $\left(\alpha_{1}^{\prime}, \alpha_{2}^{\prime}\right)$ can be reached. Now, if we have either outcome (1) or (2) then we have proven that, under $\theta_{2}$, any path for the robot starting at $\left(\mathbf{x}, \alpha_{1}, \alpha_{2}\right)$ must stick (1) or break contact (2).

Suppose, however, we have outcome (3). This outcome is not inconsistent with the negation of (F1). That is, it has not yet been shown that any path from $\left(\mathbf{x}, \alpha_{1}, \alpha_{2}\right)$ will stick or break contact. In this case, in the new slice $\left(\alpha_{1}^{\prime}, \alpha_{2}^{\prime}\right)$ we again perform the quasistatic analysis and forward project again into yet another slice. This process continues until either outcomes (1) or (2) are obtained.

More generally, the differential forward projection of $\left(\mathbf{x}, \alpha_{1}, \alpha_{2}\right)$ could consist of more than one vector. In this case, each must be taken as a forward projection direction, and in each direction we must show that outcomes (1) or (2) eventually occur. That is, the computation above must performed for each direction predicted by the quasi-static analysis, and all directions must terminate in sticking or breaking contact.

We have described how the failure mode analysis proceeds when the push-forward $H_{1}$ of the first motion $\theta_{1}$ is simply a point. It remains to generalize the discussion to the case where $H_{1}$ is a region in generalized configuration space, represented by slices. We first introduce some notation. If $C O$ denotes the generalized configuration space obstacle for $A$ due to $B$, then let $\left.C O\right|_{\alpha_{1}, \alpha_{2}}$ denote the $x-y$ slice of $C O$ at orientations $\left(\alpha_{1}, \alpha_{2}\right)$. As usual, let $\partial$ denote the obstacle edges of a set. $\epsilon$ is the slice resolution parameter. The input to this procedure is a stack $\mathcal{Q}$ of $x$-y-slices of $H_{1}$. An entry in $\mathcal{Q}$ is a triple, consisting of an $x-y$ slice $\left.H_{1}\right|_{\alpha_{1}, \alpha_{2}}$, and $\left(\alpha_{1}, \alpha_{2}\right)$, the orientations at which the slice was coniputed.

\section{Algorithm Spec}

1. Do until $\mathcal{Q}=0$ :

2. Pop the triple $\left\langle\left. H_{1}\right|_{\alpha_{1}, \alpha_{2}}, \alpha_{1}, \alpha_{2}\right\rangle$ off $\mathcal{Q}$. Let $H_{2}-\left.H_{1}\right|_{\alpha_{1}, \alpha_{2}}$.

3. Let $d F-d F_{\theta_{2}}\left(H_{2}\right)$.

4. For each $\mathbf{v}$ in $d F$ do:

5. $\quad$ Let $\left(\alpha_{1}^{\prime}, \alpha_{2}^{\prime}\right)-\epsilon \mathbf{v}+\left(\alpha_{1}, \alpha_{2}\right)$.

6. Compute $\left.\mathrm{CO}\right|_{\alpha_{1}^{\prime}, \alpha_{2}^{\prime}}$. 


$$
\begin{array}{ll}
\text { 7. } & \text { Let } H_{3}-\left.H_{2} \cap \partial C O\right|_{\alpha_{1}^{\prime}, \alpha_{2}^{\prime}} . \\
\text { ․ } & \text { If } H_{3} \neq \emptyset \text {, push the triple }\left\langle H_{3}, \alpha_{1}^{\prime}, \alpha_{2}^{\prime}\right\rangle \text { onto } \mathcal{Q} .
\end{array}
$$

Note that this is a semi-decision procedure. This is the algorithm that is actually implemented in Limited. The key step is of course the iteration step ( $\tau$ ), which we think of as

$$
\text { " } \mathrm{H}_{2}-\left.\mathrm{H}_{2} \cap \partial \mathrm{CO}\right|_{\boldsymbol{\alpha}_{1}^{\prime}, \alpha_{2}^{\prime}} "
$$

which is repeated "until $\mathrm{H}_{2}$ is null." $\left.\mathrm{CO}\right|_{\alpha_{1}^{\prime}, \alpha_{2}^{\prime}}$ is computed using the plane sweep union algorithm, as is the intersection.

\subsubsection{On the Invariance Assumption}

We have assumed that $x-y$ position of $A$ is invariant under a commanded pure rotation $\theta_{2}$. That is, commanding a pure rotation cannot result in an induced translation. On the other hand, we allow a commanded pure translation of $A$ to induce a rotation of $B$ (but not of $A$ ). These assumptions are realistic if, for example, the robot has gripped $A$ by its center shaft, and the manipulator is very stiff in the $x-y$ directions when commanding a pure rotation. In future work, relaxing this asymmetry should be explored.

\subsubsection{Quasi-Static Analysis}

We now show how the quasi-static analysis is computed. It is quite simple. We view the commanded velocity to $A$ as $\omega=(0,0, \pm 1)$. When the gears are in contact, this defines a moving constraint in the configuration space of $B$, which is a one-dimensional space. Given a contact configuration, we compute the moment arm in order to determine the direction of the constraint. The moment arm on $B$ (resp., $A$ ) is simply the vector from $B$ 's (resp. $A$ 's) center of mass to the contact point in real space. The contact point in real space can be recovered from the contact point in configuration space.

Let $r_{a}$ and $r_{b}$ denote the moment arms on $A$ and $B$, resp. Then the instantaneous velocity $v_{a}$ of the contact point on $A$, given $\omega$, is $\omega \times r_{a}$. B's direction of impending motion is given by the sign of the expression

$$
r_{b} \times \pi_{2} v_{a}=r_{b} \times \pi_{2}\left(\omega \times r_{a}\right),
$$

where $\pi_{2}$ denotes the projection of $\Re^{3}$ onto $\Re^{2}$.

We now discuss recovery of the moment arms from the contact configuration. Let $C O M_{A}$ and $C O M_{B}$ denote the centers of mass of $A$ and $B$. In these experiments, they are simply the centers of the gears. Suppose $\left(x, \alpha_{1}, \alpha_{2}\right)$ is a contact configuration. Then it lies on an algebraic surface in the generalized configuration space $\Re^{2} \times S^{1} \times S^{1}$. This surface is one of two types [Lozano-Pérez]. Let $-A$ denote the reflection of $A$ about its 
reference point. A type $(A)$ surface is generated by an edge $e_{a}$ of $-A$ and a vertex $b_{j}$ of $B$. A type (B) surface is generated by a vertex $a_{i}$ of $-A$ and an edge $e_{b}$ of $B$. Each edgevertex or vertex-edge pair is called the generator pair of the constraint surface [Donald). The edges and vertices of - $A$ (resp. B) rotate with $\alpha_{1}$ (resp., $\left.\alpha_{2}\right)$. An $\left(\alpha_{1}, \alpha_{2}\right)$-slice of the surface is found by rotating its generators by $\left(\alpha_{1}, \alpha_{2}\right)$, and taking their Minkowski sum. Hence the surface may be viewed as a parameterized line-equation, by $\left(\alpha_{1}, \alpha_{2}\right)$. The table below gives the details for recovering the moment arus from the contact configuration, contact surface in generalized configuration space, and centers of mass. We employ the following notation. For an edge $e$ or a vertex $v, e(\alpha)$ and $v(\alpha)$ respectively denote $e$ and " rotated to orientation $\alpha$. $\uplus$ denotes convolution (sometimes known as the Minkouski sum). For two sets $U$ and $V, U \oplus V=\{v+u \mid u \in U, v \in V\}$.

\begin{tabular}{|c|c|c|c}
\hline Type & Surface & $\begin{array}{c}\text { Moment arm on B } \\
r_{b}\end{array}$ & $\begin{array}{c}\text { Moment arm on A } \\
r_{a}\end{array}$ \\
\hline$A$ & $e_{a}\left(\alpha_{1}\right) \oplus b_{j}\left(\alpha_{2}\right)$ & $b_{j}\left(\alpha_{2}\right)-C O M_{B}$ & $b_{j}\left(\alpha_{2}\right)-\mathbf{x}-C O M_{A}$ \\
\hline$B$ & $a_{i}\left(\alpha_{1}\right) \oplus e_{b}\left(\alpha_{2}\right)$ & $\mathbf{x}-a_{i}\left(\alpha_{1}\right)-C O M_{B}$ & $-a_{i}\left(\alpha_{1}\right)-C O M_{A}$ \\
\hline
\end{tabular}

\subsubsection{Stiction}

What the $S$ pec algorithm does is this: it tries to show that from any slice of $H_{1}$, all paths that could possibly evolve from commanding a rotation of $A$ either (1) remain in the first slice, or (2) in some subsequent slice, stick or break contact. We have described how (2) is detected. (1) is a form of stiction; the gears do not turn. Note that (1) is a form of sticking behavior, since no motion occurs. Staying in the same slice means that $\left(\alpha_{1}, \alpha_{2}\right)$ are fixed, and $x$ and $y$ are fixed $a$ priori. Hence events (1) or (2) satisfy (F1). That is, (1) is also a form of sticking, and can be detected at run-time by the termination predicate.

Now, suppose $B$ sticks but $A$ continues to turn? This type of stiction is also no problem, since it corresponds to a differential motion $\left( \pm d \alpha_{1}, 0\right)$, which can be predicted by the differential forward projection.

\subsubsection{Failures Outside the EDR Framework}

We will momentarily digress to a practical question. It would appear that for failure mode analysis to work, non-uniform stiction would be required in our physical model of the gears. That is, it would seem that stiction would have to be impossible in the goal, but possible in $H_{1}$. This is not the assumption made in the geometrical EDR analysis and implementation. We now show that uniform stiction is in fact not an impediment to failure mode analysis, either.

It is the responsibility of the user, or of some external inference system, to ensure that (F2) holds. Suppose, however, that this inference is incorrect, and that at runtime stiction does, in fact, occur in the goal, and that the gears jam. In this case the 
run-time executive will signal failure, even though the geometrical goal has been achieved. At first glance it appears that this is incorrect. However, when we regard the goal as a combined geometrical and functional specification, it is clear that this is actually the correct termination diagnosis. That is, even though the geometrical goal has been achieved, stiction prevents the quantified difference equation (DE) on paths, goal( $\cdot$ ), from being satistied. Since something (specifically, stiction) has prevented achievement of the functional goal, it is completely correct for the run-tine executive to signal failure in this case. However, note that we regard this as serendipitous failure detection, and not as inherent in the EDR framework.

\subsubsection{Generalizations}

The specialized algorithm Spec may be generalized. The properties it exploits are (1) that certain degrees of freedom in $C$ and $J$ can be held fixed, while others may be commanded, (2) that "slices" of $C O$ can be computed, (3) set intersections can be computed, and (1) differential motion across the non-fixed degrees of freedom can be predicted using quasistatic analysis.

More precisely, the specialized algorithm generalizes to cases where we tix certain degrees of freedom $C_{f}$ and $J_{f}$, command $C_{c}$, and permit $J_{c}$ to vary (through pushing). Hence $\mathcal{G}$ is decomposed into

$$
C_{f} \times C_{c} \times J_{c} \times J_{f},
$$

$B_{e c}\left(v_{\theta_{2}}^{*}\right)$ lies in the tangent space to $C_{c}^{\prime}$, and all motion lies in the subspace $C_{c} \times J_{c}$. Using quasi-static analysis, we predict the impending motion direction, $\mathbf{v}$ which lies in the tangent space to $C_{c} \times J_{c}$. If $\alpha$ is in $C_{c} \times J_{c}$, let $H_{1} \mid \alpha$ denote a slice of $H_{1}$ at $(\boldsymbol{\alpha})$. Thus $C_{f} \times J_{f}$ are the dimensions of the slice (like $x, y$ in the gear example). Then we let $\boldsymbol{\alpha}^{\prime}-\boldsymbol{\alpha}+\boldsymbol{+} \mathbf{v}$. Finally, the iteration step is

$$
H_{3}-H_{1}|\boldsymbol{\alpha} \cap \partial C O|_{\alpha^{\prime}}
$$

The rest of the algorithm goes through mulatis mutandis. This generalization is somewhat theoretical, in that in practice the $C O$-slices, set intersections, and quasi-static analysis inay be difficult to compute for higher-dimensional problems.

\subsection{8 * Discussion: General vs. Specialized Algorithm for Failure-Mode Analysis}

T'his starred subsection may be skipped at first reading. It contains a detailed proof.

The problem with implementing algorithm Gen directly is that arbitrary time-indexed forward projections are difficult to compute. For this reason we introduced a specialized algorithm for the gear planning. While algorithms $S p e c$ and Gen appear quite different, 
in fact, Spec is simply a special case of $G e n$. The motivation behind this viewpoint is to find a uniform framework for characterizing algorithms for failure mode analysis. That is, algorithm Gen can be viewed as a high-level computational approach to failure mode analysis, while $S p e c$ is an implementation of Gen in a restricted domain. We now discuss this view of the algorithms.

Recall the definition of $\operatorname{stick}_{\theta_{2}}(\cdot)$. We now define $\operatorname{stick}_{\theta_{2}}^{*}(R)$ to be all points $x$ in $R$ such that any feasible path from $x$ consistent with the control uncertainty $B_{e c}\left(v_{\theta_{2}}^{*}\right)$, eventually sticks.

We employ the following topological notions. $\bar{U}$ denotes the closure of a set $U$. $\quad$ : $^{c}$ denotes its complement. $i(U)$ denotes its interior. $\bar{U}^{c}$ denotes the complement of the closure.

Now, consider the following step of the Spec algorithm,

7. $H_{3}-\left.H_{2} \cap \partial C O\right|_{\alpha_{1}^{\prime}, \alpha_{2}^{\prime}}$,

where $H_{2}=\left.H_{1}\right|_{\alpha_{1}, \alpha_{2}}$. This step is equivalent to

$$
H_{3}-H_{2}-i\left(\left.C O\right|_{\alpha_{1}^{\prime}, \alpha_{2}^{\prime}}\right)-\overline{\left.C O\right|_{\alpha_{1}^{\prime}, \alpha_{2}^{\prime}}}
$$

where the set difference operator - associates to the left. Now, the set

$$
H_{2} \cap i\left(\left.\mathrm{CO}^{\prime}\right|_{\alpha_{1}^{\prime}, \alpha_{2}^{\prime}}\right)
$$

corresponds to all configurations $\left(x, \alpha_{1}, \alpha_{2}\right)$ in the planar slice $\left(\alpha_{1}, \alpha_{2}\right)$ such that under $\theta_{2}$, any path from $\left(\mathbf{x}, \alpha_{1}, \alpha_{2}\right)$ will stick before reaching $\left(\alpha_{1}^{\prime}, \alpha_{2}^{\prime}\right)$ if $\mathbf{x}$ is kept tixed. That is, it is configurations such that sticking will occur from $\left(\mathbf{x}, \alpha_{1}, \alpha_{2}\right)$ between $\left(\alpha_{1}, \alpha_{2}\right)$ and $\left(\alpha_{1}^{\prime}, \alpha_{2}^{\prime}\right)$.

Below, we argue that the set $H_{2} \cap i\left(\left.C O\right|_{\alpha_{1}^{\prime}, \alpha_{2}^{\prime}}\right)$ in algorithm Spec corresponds in a quite precise fashion to $\operatorname{stick}_{\theta_{2}}(\partial F)$ in algorithm Gen. We see this as follows:

The following step of the Gen algorithm, ${ }^{24}$

2. $H_{3} \leftarrow \partial F-\operatorname{stick}_{0_{2}}(\partial F)$.

is equivalent to

$$
H_{3}-F-\overline{C O^{c}}-\operatorname{stick}_{\theta_{2}}(\partial F) \text {. }
$$

Now, it is possible to modify Gen as follows. Let

$$
F_{2}=F_{\theta_{2}, \Delta \iota}\left(H_{1}-s t i c k_{\theta_{2}}^{*}\left(H_{1}\right)\right) \text {. }
$$

\footnotetext{
${ }^{24}$ We have lexicographically substituted $H_{3}$ for $H_{2}$ throughout algorithm $G e n$ to facilitate the comparison with Spec.
} 
Then we can replace the assignment (11) by eq. (12) and still have Gen be correct:

$$
H_{3}-F_{2}-\overline{C O}^{c}
$$

We wish to compare the step $(12)$ of the thus modified Gen with the step of Spec given in eq. (10). In essence, we wish to show that eq. (10) is in some sense a "conservative" approximation to eq. (12), and hence conclude that algorithm S'pec is simply a special case of algorithm Gen.

We must introduce some notation to compare eqs. (10) and (12). For a set $V$ in $\Re^{2}$, we denote the set

by

$$
V \times\left\{\left(\alpha_{1}, \alpha_{2}\right)\right\}
$$

$$
V \times\left(\alpha_{1}, \alpha_{2}\right) .
$$

Now, $H_{1}$ is a subset of $\mathcal{G}$. A slice of it $\left.H_{1}\right|_{\alpha_{1}, \alpha_{2}}$ lies in the "plane" $\Re^{2} \times\left(\alpha_{1}, \alpha_{2}\right)$. Let us denote its projection into $\Re^{2}$ by $\left.\pi_{2} H_{1}\right|_{\alpha_{1}, \alpha_{2}}$. Finally, for an arbitrary set $U$ in generalized configuration space, let $\left.U\right|_{\alpha_{1}, \alpha_{2}}$ denote an $\left(\alpha_{1}, \alpha_{2}\right)$-slice of it, that is,

$$
\left.{ }^{I}\right|_{\boldsymbol{\alpha}_{1}, \boldsymbol{\alpha}_{2}}=U \cap\left(\Re^{2} \times\left(\boldsymbol{\alpha}_{1}, \boldsymbol{\alpha}_{2}\right)\right) .
$$

Claim: Eq. (10) is a conservative approximation to eq. (12) in each slice.

Proof: First, we obviously have

$$
\left.C O\right|_{\alpha_{1}^{\prime}, \alpha_{2}^{\prime}} \subset C O
$$

Next, we need only show that

$$
\left.\left(F_{\theta_{2}, \Delta t}\left(\left.H_{1}\right|_{\alpha_{1}, \alpha_{2}}\right)\right)\right|_{\alpha_{1}^{\prime}, \alpha_{2}^{\prime}} \subset \pi_{2}\left(\left.H_{1}\right|_{\alpha_{1}, \alpha_{2}}\right) \times\left(\alpha_{1}^{\prime}, \alpha_{2}^{\prime}\right)
$$

and

$$
\left.H_{1}\right|_{\alpha_{1}, \alpha_{2}} \cap \pi_{2}\left(i\left(\left.C O\right|_{\alpha_{1}^{\prime}, \alpha_{2}^{\prime}}\right)\right) \times\left(\alpha_{1}, \alpha_{2}\right) \sqsubset \operatorname{stick}_{\theta_{2}}^{*}\left(\left.H_{1}\right|_{\alpha_{1}, \alpha_{2}}\right) .
$$

Eqs. (14) and (15) are definitional. Now, suppose that configuration $z \in i\left(\left.C O\right|_{\alpha_{1}^{\prime}, \alpha_{2}^{\prime}}\right)$. 'Then clearly $\left.z \notin F\right|_{\alpha_{1}^{\prime}, \alpha_{2}^{\prime}}$. Hence we have

$$
\begin{aligned}
\left.H_{3, G e n}\right|_{\alpha_{1}^{\prime}, \alpha_{2}^{\prime}} & =\underbrace{\left.\left(F_{\alpha_{2}, \Delta t}\left(H_{1}-s t i c k_{\theta_{2}}^{*}\left(H_{1}\right)\right)\right)\right|_{\alpha_{1}^{\prime}, \alpha_{2}^{\prime}}}_{\bigcap}-\overline{\left.C O\right|_{\alpha_{1}^{\prime}, \alpha_{2}^{\prime}}} \\
H_{3, \text { Spec }} & =\overbrace{\left(\pi_{2} H_{2} \times\left(\alpha_{1}^{\prime}, \alpha_{2}^{\prime}\right)\right)-i\left(\left.C O\right|_{\alpha_{1}^{\prime}, \alpha_{2}^{\prime}}\right)}^{n}-\overline{\left.C O\right|_{\alpha_{1}^{\prime}, \alpha_{2}^{\prime}}} .
\end{aligned}
$$


Note that as a consequence, we may expect that Spec is less likely than (ien to terminate.

\section{Weak EDR Theory, Strategy Equivalence, and the Linking Condition}

\subsection{Reachability and Recognizability Diagrams}

We now introduce a type of diagram which permits notation of reachability and recognizability. These diagrams are a powerful tool for compactly expressing motion strategies. They greatly aid the development of concise and readable proofs.

Suppose we are given a start region $R$, a goal $G$, and a motion $\theta$. We construct the EDR region $H$. Then under sticking termination, all motions from $R$ will terminate in $G$ or $H$. That is, the push-forward of the motion $\theta$ from $R$ is contained in $G \cup H$ :

$$
\operatorname{push}_{\theta}\left(F_{\theta}(R)\right) \subset G \cup H \text {. }
$$

Whenever (16) is true, we write this by the following reachability diagram,

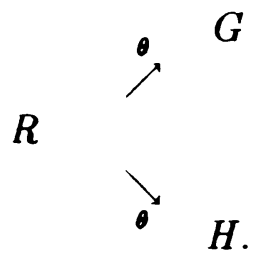

$H$.

Suppose that $G$ and $H$ are distinguishable using sensors. Then $\theta$ is an EDR strategy from $R$, and we have

$$
R=P_{0, R}(\{G, H\}) .
$$

Whenever (18) holds, we write this by the following recognizability diagram,

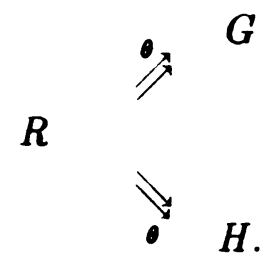

The reachability diagram (17) is an equivalent notation for the reachability termination condition (16). The recognizability diagram (19) is equivalent notation for the recognizability termination condition (18). Single arrows $(\longrightarrow)$ denote reachability whereas double arrows ( $)$ denote recognizability. If and only if (16) is true, we say that the 
correspondingly reachability diagram (17) holds. If and only if (18) is true, we say that the correspondingly recognizability diagram (19) holds. A diagram is said to hold tautol. ogously when it is true without additional conditions or suppositions.

The nice thing about sticking termination, as discussed in chapter II, is the following property:

Theorem: Let $R$ be a start region, $\theta$ a motion, and $G$ a goal. Construct the EDR region $H$ for $R, \theta$, and $G$. Then with sticking termination the reachability diagram (17) holds tautologously.

Now, in diagrams (17) and (19) we have labeled all the arrows. In the future, when this would clutter the diagrams, we will label only the top arrow and adopt the convention that all arrows aligned below it have the same label.

\subsection{More General Push-Forwards}

Hence the chief advantage with sticking termination is that (17) is always true. In this chapter, we will generally assume that either sticking termination is employed, or, if more general termination predicates are allowed, then the truth of the reachability diagram (17) can be determined through restrictions on time and history, as described in chap. We now digress briefly, however, to describe how this discussion generalizes for more general termination predicates.

In an appendix, we define a more general push-forward, $F_{* \theta}(R)$, which denotes all configurations at which the motion $\theta$ can terminate given more general termination predicates. When more general termination predicates than sticking are considered, then the condition (16) must be replaced by

$$
F_{* \theta}(R) \subset G \cup H .
$$

When (20) holds, we may then write the equivalent reachability diagram (17).

However, with more general termination conditions, (17) does not hold tautologously. For example, with time-termination and the approximate push-forward described in sec. 5.3 , a motion could (a priori) terminate without sticking yet within the weak preimage. In such cases, it must be the responsibility of the planner to verify that all motions terminate in $G \cup H$.

The first difference between the sticking push-forward push $(\cdot)$ and the general pushforward $F_{*}(\cdot)$ is that $F_{*}(\cdot)$ depends on the start region for the motion, while push $(\cdot)$ does not. That is, $F_{*}(\cdot)$ depends on history (and possibly time) whereas push $(\cdot)$ does not.

Now, a motion sequence is a reachability or recognizability diagram of the form:

$$
R_{n} \stackrel{\theta_{n}}{\longrightarrow} R_{n-1} \stackrel{\theta_{n-1}}{\longrightarrow} \cdots \stackrel{\theta_{2}}{\longrightarrow} R_{1} \stackrel{\theta_{1}}{\longrightarrow} R_{0}=G
$$


The second chief difference between the a priori sticking push-forward push(.) and the general push-forward $F_{*}(\cdot)$ is that the action of push $(\cdot)$ on a motion sequence $(21)$ is functorial, while $F_{*}(\cdot)$ is not. The non-functoriality of $F_{*}(\cdot)$ is a consequence of its history dependence.

\subsection{Weak EDR Theory}

We now make the following natural refinement of our termination predicate. Suppose the termination predicate is given some finite collection of goals $\left\{G_{\beta}\right\}$ in a distinguishable union. Then the goals $\left\{G_{\beta}\right\}$ are of course partially ordered by containment. We assume that the termination predicate returns the smallest goal (with respect to containment) if at termination time the actual configuration of the robot is known to lie within two or more goals. (A technical point: if two or more goals overlap, we augment the collection with a new goal which is their intersection).

Now, whenever the reachability diagram (17) holds (which it always does with sticking termination), then we have the following:

$$
R=P_{\theta, R}(\{G, H, G \cup H\}) .
$$

This is trivial to show; on termination, the termination predicate will return $G$ or $H$ if it can, otherwise it will return $G \_H$. In particular, it will return $G$ or $H$ in preference to $G \cup H$.

Thus we can write the following recognizability diagram, which is equivalent to $(22)$ :

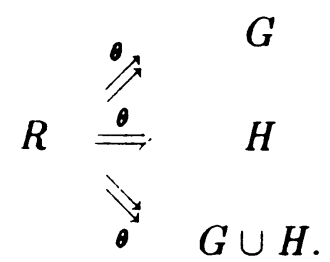

(23) is called the Weak EDR Recognizability Diagram for $G, H$, and $\theta$. (19) is called the Strong EDR Recognizability Diagram. (17) is called the Reachability Diagram.

Theorem: Let $R$ be a start region, $\theta$ a motion, and $G$ a goal. Construct the EDR region $H$ for $R, \theta$, and $G$. Then with sticking termination the weak EDR diagram (23) holds tautologously.

Up to now, in previous chapters, we have described the strong EDR theory. This section has introduced the weak EDR theory. It may not appear useful at first glance. However, in the next section we will see that these one-step weak EDR strategies-which are in effect always available-may under certain conditions be chained together to make a multi-step plan very like a strong EDR strategy. 
The key idea behind the weak EDR theory is: given a collection of goals $\left\{G_{j}\right\}$ (possibly including $H$ ), we consider all unions of the subcullections to get some measure of weakest recognizability.

\subsection{Strategy Equivalence}

A one-step weak EDR strategy is not very interesting. In particular, we can always obtain one! Surprisingly, it is possible to define a way of coupling two weak, one-step EI)R strategies together to make a two step strategy which has many of the characteristics of strong EDR. In particular, we will develop a way of making precise the idea that the two weak EDR steps can be combined to make a two-step strategy that is "equivalent" to a one-step strong EDR strategy.

Suppose the commanded motions of the two weak EDR steps are $\theta_{1}$ and $\theta_{2}$. The essence of this "equivalence" lies in disambiguating a previous motion's $\left(\theta_{1}\right.$ 's) result without destroying the goal state.

Now, let $R$ be the start region, and $G$ the goal as usual. Assume without loss of generality that $G$ is contained within the forward projection of $R$ under $\theta_{1}$. Let

$$
R_{1}=R \cap P_{\theta_{1}, F_{\mathbf{O}_{1}}(R)}(G) .
$$

Now, we have the recognizability diagrams



where $H^{\prime}$ is the EDR region for motion $\theta_{2}$.

The question is, how can we link together motions $\theta_{1}$ and $\theta_{2}$ into a two-step EDR strategy? The first condition we require of such a two-step strategy is as follows: once $\theta_{1}$ has reached $G, \theta_{2}$ should preserve this state and "add" recognizability. That is, $G$ is a "fixed-point" under $\theta_{2}$. This is given by the following diagram:

Definition: The fixed-point diagram is

$$
\operatorname{push}_{0_{1}}(G) \stackrel{\theta_{2}}{=} G
$$

When the fixed-point diagram (26) holds, (25) admits the following reachability and recognizability diagram: 


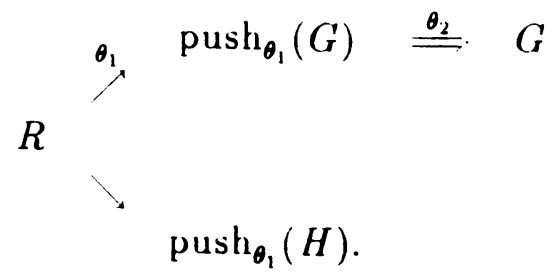

It remains to ensure that good EDR behavior occurs when $\theta_{2}$ is executed from push $_{\theta_{1}}(H)$. Now, think of $\theta_{1} * \theta_{2}$ as the composite strategy formed by executing motion $\theta_{1}$ followed by $\theta_{2}$. We wish to find additional conditions which, together with (25), will admit both the fixed-point diagram (26) and a strong EDR diagram,

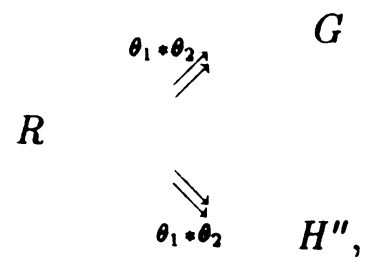

for some $H^{\prime \prime}$ (see below). Together with the weak EDR diagram (25) (which is tautol(gously true for sticking termination), the additional conditions below, which we will call the linking conditions, are necessary and sufficient for defining an equivalence between two "linked" weak EDR strategies and a single-step strong EDR strategy, whose recognizability diagram is given by (19), (substituting $\theta_{1}$ for $\theta$ ). Henceforth, let $\theta=\theta_{1}$.

Definition: If the fixed-point diagram (26) holds and if (25) admits a strong EDR diagram (28) in which

$$
H^{\prime \prime}=\left\{H^{\prime}\right\},
$$

then the motion strategy $\theta_{1} * \theta_{2}$ is said to be strongly equivalent to a strong EDR strategy with recognizability diagram (19).

An example of such a strategy is the two-step peg-in-hole insertion plan with model error, figs. 4-66.

Definition: If the fixed-point diagram (26) holds and if (25) admits a strong E'DR diagram (28) in which

$$
H^{\prime \prime}=\left\{H^{\prime}, H^{\prime} \cup G\right\},
$$

then the motion strategy $\theta_{1} * \theta_{2}$ is said to be weakly equivalent to a strong EDR strategy with recoynizability diagram (19).

Note that we define (strong or weak) equivalence using (19) with $\theta=\theta_{1}$, not with $\theta=\theta_{1} * \theta_{2}$. The reason for this is as follows. If $\theta_{1} * \theta_{2}$ satisfies the weak equivalence 
condition (30) and the fixed-point diagram (26), then after termination, we are assured that the outcome of $\theta_{1}$ has been completely diagnosed. That is, the run-time executor knows whether or not $\theta_{1}$ terminated in success or failure. However, it is not necessarily true that the outcome of $\theta_{2}$ is completely diagnosed. 'This ocrurs in the worst case, if $H^{\prime} \cup G$ is recognizably attained. We discuss this point in some detail below.

The following gives an implicit definition of linking conditions:

Definition: Let $H^{\prime \prime}$ be chosen for either strong or weak equivalence, as in (29) or (30). The linking conditions are necessary and sufficient conditions for (25) to admit a fixedpoint diagram (26) and a strong EDR diagram (28).

It remains to show, of course, that linking conditions exist for strong or weak equivalence. We will momentarily postpone the derivation of the linking conditions in order to describe what the linking should effect.

Once "linked," two one-step weak EDR plans should admit the strong EDR diagram (28). The claim is that $(28)$ is in some sense "equivalent" to the strong EDR diagram (19). How is this possible?

(19) indicates that the run-time executor can disambiguate the success or failure of motion $\theta_{1}$. The same is true of strategy $\theta_{1} * \theta_{2}$ in (28). Here are the possible results of executing $\theta_{1} * \theta_{2}$ when the steps $\theta_{1}$ and $\theta_{2}$ are properly "linked:"

1. $G$ is achieved and recognized at termination. In this case, either (i) $\theta_{1}$ achieved $G$ and the run-time executive may not have recognized it, but $\theta_{2}$ disambiguated the result while still terminating within $G$. Alternatively, (ii) $\theta_{1}$ failed, reaching $H$, and $\theta_{2}$ subsequently achieved $G$ from $H$.

2. $H^{\prime}$ is achieved and recognized at termination. In this case, $\theta_{1}$ is known to have failed, and the robot is known to be outside $G$.

(1) and (2) are the only outcomes given strong equivalence. With weak equivalence, a third outcome in also possible:

3. $G \cup H^{\prime}$ is achieved and recognized at termination. In this case, $\theta_{1}$ is known to have failed.

Thus the key is that $\theta_{2}$ does not corrupt the goal state; that is, $G$ is a fixed point under $\theta_{2}$. The desirability of outcomes (1) and (2) are clear. One might ask, what good is weak equivalence? Why would anyone want outcome (3)? The answer is: in one-step strong EDR (19), the run-time executor can (a) disambiguate the result of motion $\theta_{1}$, and (b) in case of failure, know that the robot is not in the goal. In weak equivalence, we 
have (a) but not (b). That is, in outcome (3), we have completely diagnosed the result if motion $\theta_{1}$, although in the process, we may have accidentally moved into the goal. That is, we may indicate failure when we have, in fact, succeeded. However, we will never indicate success unless it is certain. In short, when linked, $\theta_{1} * \theta_{2}$ is "conservative" about declaring success.

\subsection{The Linking Conditions}

We now derive the linking conditions. Let

$$
\begin{aligned}
F_{\theta_{1}} & =F_{\theta_{1}}(R) \\
R_{1} & =R \cap P_{\theta_{1}, F_{\theta_{1}}}(G) \\
\operatorname{push}_{\theta_{1}} & =\operatorname{push}_{\theta_{1}}(G \cup H) \\
F_{\theta_{2}} & =F_{\theta_{2}}\left(\operatorname{push}_{\theta_{1}}\right) \\
R_{2} & =\operatorname{push}_{\theta_{1}} \cap P_{\theta_{2}, F_{\theta_{2}}}(G) .
\end{aligned}
$$

The overloading notation for push $\boldsymbol{\theta}_{1}$ is symmetric with that for preimages and forward projections: both the map and its image are denoted by the same symbol. The discussion of linking conditions assumes sticking termination. However, the derivation goes through mutatis mutandis for more general termination conditions, if we let

$$
\text { push }_{\theta_{1}}=F_{* \theta_{1}}(R) \text {. }
$$

It remains, however, to extend the linking-conditions for time-indexed forward projections.

We now demonstrate our claim that linking conditions exist.

Definition: The condition (LO) is

$$
G \cap \text { push }_{\theta_{1}} \subset R_{2} \text {. }
$$

Here is the motivation behind (LO). (LO) says that whenever motion $\theta_{1}$ terminates in the goal $G$, then the state is inside the preimage of $G$ under the next motion $\theta_{2}$. The intent of (L0) is to admit the fixed-point diagram (26).

Claim: (LO) implies the fixed-point diagram (26).

Proof: The preimage equation for (26) is

$$
P_{\theta_{2}, \text { push }_{0_{1}}(G)}(G)=\operatorname{push}_{\theta_{1}}(G) .
$$

This preimage is taken with respect to a smaller start region than $R_{2}$.

Note however that the converse is false. (LO) is stronger than the fixed-point diagram (26), since the preimage $R_{2}$ is taken with respect to the entire forward projection under $\boldsymbol{\theta}_{\mathbf{2}}$. 
Claim: Linking conditions exist, and, in particular, (LO) is a linking condition. Proof: Suppose (LO) holds. This yields the following reachability and recognizability diagram:

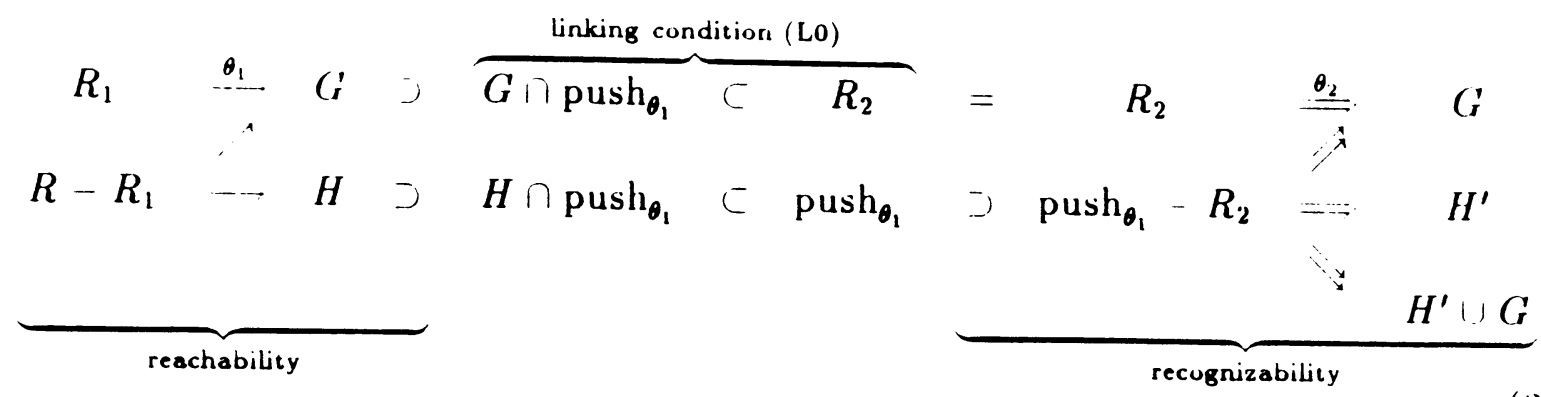

To see that diagram (31) demonstrates weak equivalence, we use a technique like "diagram chasing" (see, eg., [Hungerford)). Assume (L0) holds. Starting from $R_{1}, \theta_{1}$ effects a motion reaching $G$. This motion in fact terminates in $G$ / push , $_{\theta_{1}}$. Since by (L0) $G \cap$ push $_{\theta_{1}}$ is within $R_{2}, \theta_{2}$ then effects recognizable termination in $G$.

On the other hand, if the motion begins in $R-R_{1}$, then $\theta_{1}$ effects a motion reaching either $G$ or $H$. If $G$ is reached, then $\theta_{2}$ will eventually effect recognizable termination in $G$, by the argument immediately above. If $H$ has been reached, then the motion $\theta_{1}$ will in fact terminate at some point $z$ in $H \cap$ push $_{\theta_{1}}$. Then there are two cases. Case (i): $z \in R_{2}$. Since the preimage $R_{2}$ is constructed with respect to the entire forward projection of push $_{\theta_{1}}$, motion $\theta_{2}$ will next effect recognizable termination in $G$. Case (ii): $z \notin R_{2}$. In this case, motion $\theta_{2}$ will effect recognizable termination in one of $\left\{G, H, H^{\prime} \cup G\right\}$.

We conclude the process by "forgetting" all the intermediate steps, and renaming them to $\theta_{1} * \theta_{2}$. First, observe that the fixed-point diagram (26) holds. Next, to see that (31) admits an EDR diagram (28) in which (30) holds, we remember only the start region $R$ and the "results" $G, H^{\prime}$, and $H^{\prime} \cup G$. Diagram chasing shows that these may be joined with recognizability arrows as in (28).

Thus the diagram (31) demonstrates weak equivalence. For strong equivalence, we remove $H^{\prime} \cup G$ as an outcome of $\theta_{2}$. Note that the linking condition is not a tautology. However, note that all the other subset relations and the equality in (31) are tautologous.

In the future, we will leave similar diagram-chasing arguments to the reader. We may thus conclude that

Theorem: The linking condition (LO) is a necessary and sufficient condition for weak equivalence of $\theta_{1} * \theta_{2}$ to a one-step strong EDR strategy.

Proof: The claims above have demonstrated sufficiency. It remains to show (L0) is necessary. Suppose (L0) is false, but (26) still holds. (This is the interesting case, for if (26) does not hold, then equivalence cannot possibly follow). (26) says that when 
the motion is known to start within push $_{\theta_{1}}(G)$, then it can be guaranteed to terminate recognizably in $G$. The antecedent is a precondition for success of the motion. After $\theta_{1}$, however, this precondition may be false: even if $\theta_{1}$ reaches $G$, it is only known to have reached push $\theta_{\theta_{1}}$. In particular, $(26)$ says nothing about what happens when $\theta_{2}$ is executed from $H$. (LO), on the other hand, says that termination in $G$ can be recognized no matter where $\theta_{2}$ originates in push $\theta_{1}$.

Now, we can derive some equivalent linking conditions that are somewhat simpler in form. Let

$$
R_{2}^{*}=R_{2} \mapsto G
$$

Definition: The linking conditions (L1) and (L2) are

$$
\begin{array}{rlc}
G \cap \text { push }_{\theta_{1}} & = & R_{2}^{*} \\
H \cap \text { push }_{\theta_{1}} & =\text { push }_{\theta_{1}}-R_{2}^{*}
\end{array}
$$

These linking conditions admit the reachability and recognizability diagram

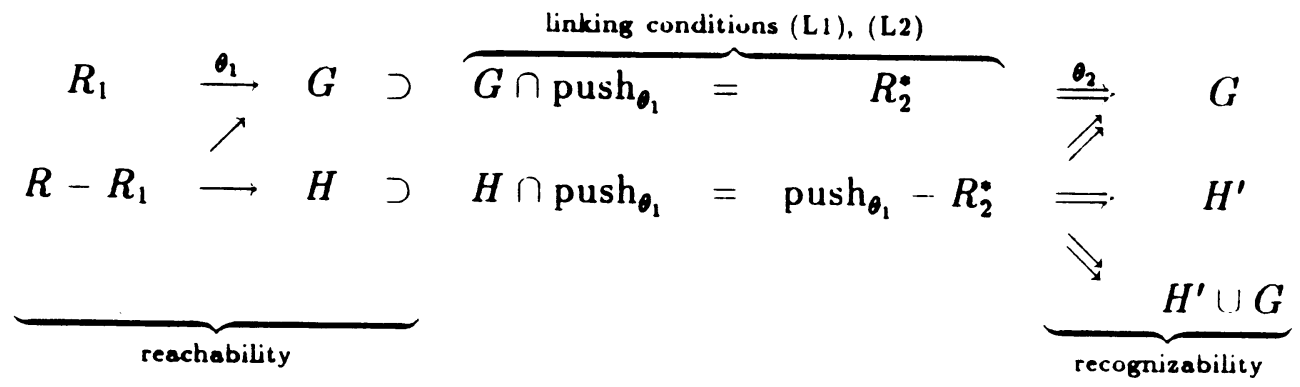

Cominents: Let

$$
P_{\theta_{2}}=P_{\theta_{2}, F_{0_{2}}\left(\text { push }_{0_{1}}(G)\right)}(G)
$$

so $R_{2}=$ push $_{\mathrm{O}_{1}}$ / $P_{\mathrm{O}_{2}}$. Note that (L2) is not tautologous, for we can have $x \in G, x \notin P_{\theta_{2}}$ if $(\mathrm{L} 1)$ is false. Therefore $x \in$ push $_{0_{1}}-R_{2}^{*}$ and $x \notin$ push $_{\theta_{1}} \cap H$.

Lemma. The linking conditions $(L 1)$ and $(L 2)$ are equivalent.

Proof: (L1) implies (L2). Suppose (L1). Let $x \in H \cap$ push $_{0_{1}} \cdot x \in R_{2}^{*}$ implies $x \in G$. Therefore $x \notin H$ is a contradiction. Therefore $x \in$ push $_{\theta_{1}}-R_{2}^{*}$.

Now let $x \in$ push $_{0_{1}}-R_{2}^{*}$. Therefore $x \notin G \cap$ push $_{\theta_{1}}$ । $P_{\theta_{2}}$. Therefore $x \notin G$ or $x \notin P_{\theta_{2}}$. In the former case, $x \in H$. In the latter, suppose that $x \in$ push $_{\theta_{1}}$ and $x \in G$ and $x \notin P_{\theta_{2}}$. But by (L1), $x \in G \cap$ push $_{0_{1}}$ implies $x \in P_{\theta_{2}}$, a contradiction.

(L1) if (L2). Let $x \in G \cap$ push $_{0_{1}}$. Show $x \in R_{2}^{*}$. We need only show that $x \in P_{\theta_{2}}$. Now, $x \notin P_{\boldsymbol{O}_{2}}$ implies $x \in H \cap$ push $_{\boldsymbol{O}_{1}}$, a contradiction. Now let $x \in R_{2}^{*}$. Therefore, $\boldsymbol{x} \in \boldsymbol{G} \cap$ push $_{\boldsymbol{\theta}_{1}}$. 
Lemma: The linking conditions (LO) and (LI) are equivalent.

Proof: (LU) implies (LI). Suppose (L0), i.e., $G \cap$ push $_{\theta_{1}} \subset R_{2}$. Show $G \cap$ push $_{\theta_{1}}=R_{2}^{*}=$ $G \cap R_{2}$.

Let $x \in G \cap$ push $_{\theta_{1}}$. Now, (L0) implies that $x \in R_{2}$. Therefore $x \in G$ and $x \in R_{2}$. Hence $x \in R_{2}^{*}$.

Let $x \in R_{2}^{*}$. Therefore $x \in G \cap R_{2}$. Hence $x \in G \cap$ push $_{\theta_{1}} \cap P_{\theta_{2}}$, i.e., $x \in G \backslash$ push $_{\theta_{1}}$. (LO) if $(L 1)$ is trivial.

Theorem: The following linking conditions are equivalent:

$$
\begin{array}{llc}
G \backslash \operatorname{push}_{\theta_{1}} & \subset & R_{2} \\
G \| \operatorname{push}_{\theta_{1}} & = & R_{2}^{*} \\
H 门 \operatorname{push}_{\theta_{1}} & = & \text { push }_{\theta_{1}}-R_{2}^{*}
\end{array}
$$

\section{6 * Beyond the Fixed-Point Restriction}

In the discussion above, we have required that the goal was a fixed point under motion $\theta_{2}$. We now discuss how to relax this restriction. In particular, it is possible to extend the notions of strategy equivalence, and the linking conditions, to the case where a subgoal $G_{1}$ is in fact the preimage of the actual, or final goal, $G_{0}$, under $\theta_{2}$. Thus $G_{1}$ is no longer the fixed point of $\theta_{2}$, but rather the preimage of $G_{0}$. This section is somewhat technical and may be skipped at first reading. We regard relaxing the fixed-point restriction as a digression. The subsequent material may be understood even if this section is omitted, however, the reader may wish to bear in mind that such a generalization does, in fact, exist.

We consider the situation where from $R, \theta_{1}$ may attain $G_{0}$ or $G_{1}$, where " $G_{1}$ $P_{\theta_{2}}\left(G_{0}\right)$." However, $G_{1}$ may not be distinguishable from $G_{0}$ under $\theta_{1}$. Thus the three reachability results of $\theta_{1}$ are $G_{0}, G_{1}$, or $H_{1}$, where $H_{1}$ is the EDR region for $\theta_{1}$ when we view the goal as $G_{0} \cup G_{1}$.

To define strategy equivalence in the non-fixed-point case, we first generalize the fixed-point diagram (26) as follows.

Definition: The generalized fixed-point diagram is

$$
\operatorname{push}_{0_{1}}\left(G_{0} \cup G_{1}\right) \stackrel{\theta_{2}}{\Longrightarrow} G_{0} \text {. }
$$

Next, we modify the definitions of strategy equivalence and the linking conditions to require that the generalized fixed-point diagram (33) hold in place of the old fixed-point diagram (26). To avoid confusion, we will call (26) the simple fixed-point condition.

Now, we let 


$$
\begin{aligned}
R_{1} & =R \\
\text { push }_{\theta_{1}} & =F_{* \theta_{1}}(R) \\
F_{\theta_{2}} & =P_{\theta_{1}, F_{\theta_{1}}}\left(G_{0}\left(\operatorname{push}_{\theta_{1}}\right)\right. \\
P_{\theta_{2}} & =P_{\theta_{2}, F_{\theta_{2}}}\left(G_{0}\right) \\
R_{2} & =\text { push }_{\theta_{1}} \cap P_{\theta_{2}} .
\end{aligned}
$$

Next, define

$$
R_{2}^{j}=G_{j} \cap R_{2}
$$$$
(j=0,1)
$$

It is possible to generalize the definition of $R_{2}^{j}$ and the linking conditions to more than two subgoals $\left\{G_{j}\right\}$. We would do this by writing $(\forall j)$ in place of $(j=0,1)$.

We already know one linking condition:

$$
P_{\theta_{2}} \supset G_{0} \cup G_{1} \text {. }
$$

In addition, we can derive the following linking conditions. Recall $H_{1}$ is the EDR region for motion $\theta_{1}$, viewing the goal of $\theta_{1}$ as $G_{0} \cup G_{1}$.

$$
\begin{array}{ccccc}
\text { push }_{\theta_{1}} \cap G_{j} & = & R_{2}^{j} \\
\text { push }_{\theta_{1}} \sqcap H_{1} & =\operatorname{push}_{\theta_{1}}-\bigcup_{j} R_{2}^{j} .
\end{array}
$$

Comments: Clearly we have $\left(L 1^{\prime}\right)$ implies $\left(L 2^{\prime}\right)$. However I have not been able to prove the converse true. I suspect it is false, since $G_{0}$ may intersect $G_{1}$, and $H_{2}$, the EDR region for $\theta_{2}$, may intersect $G_{1}$, etc.

Finally, note that all three linking conditions, $\left(L 1^{\prime}, L 2^{\prime}, L 3\right)$ are required for the composition $\theta_{1} * \theta_{2}$ to admit an equivalent strong EDR diagram. This points out the chief theoretical advantage of strategy equivalence with the simple fixed-point condition (26). With the simple fixed-point condition, the linking conditions (LO), (L1) and (L2) were found to be equivalent. With the generalized fixed point condition (33), not only do the corresponding linking conditions $\left(L 1^{\prime}\right)$ and $\left(L 2^{\prime}\right)$ appear to be inequivalent, but we also require the additional independent condition $(L 3)$. While it is gratifying that our key concept--composing two weak EDR strategies via linking conditions to admit strategy equivalence-in fact generalized to the non-fixed-point case, the generalization, unfortunately, is correspondingly more complicated.

\subsection{What Good is Weak Equivalence?}

We now pose the following question. Why is 


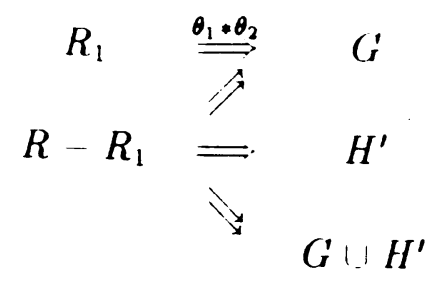

any better than



(35) is simply the weak EDR diagram for motion $\theta_{1}$. It always holds (given the reachability diagram). (34) is the equivalent recognizability diagram for $\theta_{1} * \theta_{2}$ when a linking condition is satisfied. That is, (34) is obtained through weak equivalence. Why is (34) stronger than (35), and would one prefer (34) to (35)?

Here is our answer. push $_{\theta_{1}}(G)$ is a fixed-point of $\theta_{2}$. Therefore, nothing is "lost" by $\theta_{2}$. $\theta_{2}$ serves to disambiguate the result of $\theta_{1}$, without polluting the state. Second, note that $\theta_{1} * \theta_{2}$ is "conservative" about declaring success. It is as if we used $\theta_{2}$ to convert the reachability diagram

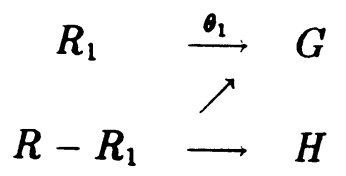

into the recognizability diagram

$$
\begin{gathered}
R_{1} \stackrel{G \stackrel{\theta_{1} * \theta_{2}}{\rightleftharpoons}}{\stackrel{\text { "Win" }}{\Longrightarrow}} \text { "Lose, but knowing } \theta_{1} \text { did not achieve } G . "
\end{gathered}
$$

Mure precisely, the "lose" states are

$H^{\prime} \approx \theta_{1}$ did not achieve $G$, and now the robot is outside of $G$.

$G \cup H^{\prime} \approx \theta_{1} \operatorname{did}$ not achieve $G$, and now we might be in $H^{\prime}$, but can't guarantee that we're outside of $G$.

On the other hand (35), achieving $G \cup H$ after $\theta_{1}$ only tells us that started in $R-R_{1}$, and does not tell us the result of motion $\theta_{1}$. 


\subsection{Application: Failure Mode Analysis in the Gear Experi- ment}

We now discuss how the failure mode analysis used to generate motion $\theta_{2}$ in the gear dornain may be viewed using the weak EDR theory.

In the gear meshing plan, $\theta_{1}$ is a pure translation, and $\theta_{2}$ is a pure rotation. The goal is a fixed point under $\theta_{2}$. Consider $(32)$. In the gear plan, the reachability arc

$$
R-R_{1} \stackrel{\theta_{1}}{\theta^{\prime}}
$$

is present, but the arc

$$
{ }_{22} \quad G
$$

is not. That is, it is possible to serendipitously achieve the goal under translation but not rotation. The linking conditions are satisfied. Now, is the outcome $G \cup H^{\prime}$ possible? Failure mode analysis yields the answer: No. In this case, $\theta_{1} * \theta_{2}$ is strongly equivalent to a one-step strong EDR strategy

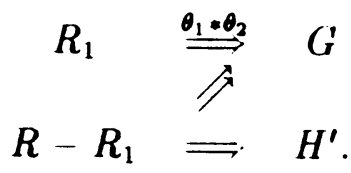

The full reachability and recognizability diagram for the gear plan is given by

$$
\begin{aligned}
& R_{1} \stackrel{\theta_{1}}{\longrightarrow} G \supset \overbrace{G \cap \text { push }_{\theta_{1}}=}^{\text {linking conditions (L1), (L2) }} \stackrel{R_{2}^{*}}{\stackrel{\theta_{2}}{\longrightarrow}} G \\
& R-R_{1} \longrightarrow H \quad H \quad H \cap \operatorname{push}_{\theta_{1}}=\operatorname{push}_{\theta_{1}}-R_{2}^{*} \Longrightarrow H^{\prime} \\
& \text { reachability }
\end{aligned}
$$

\subsection{Discussion and Review}

We now discuss the relationship between push-forward algorithms, failure-mode analysis, and the weak EDR theory. Recall the diagram (32): 


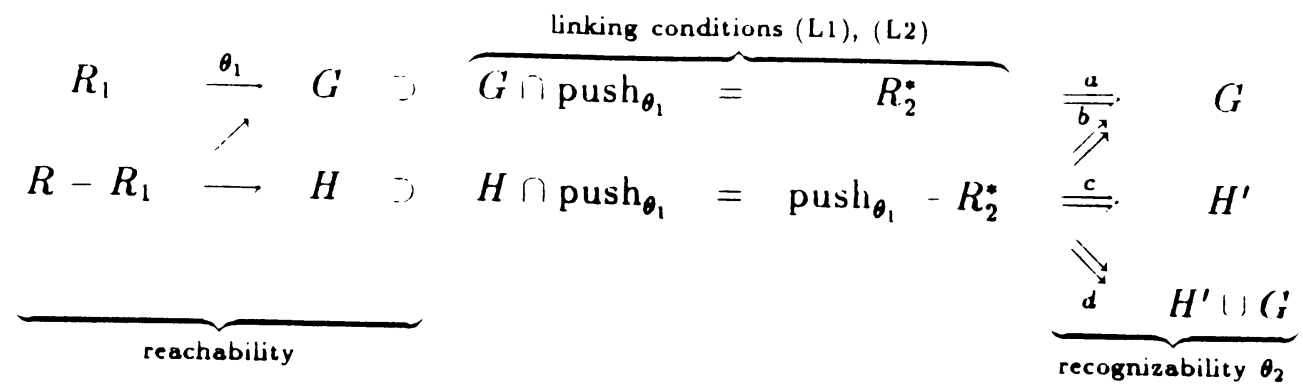

(41) is the full reachability and recognizability diagram for weak equivalence. The arrows (a)-(d) all correspond to motion $\theta_{2}$; we have labeled them so as to be able to refer to them in the discussion.

Failure Mode Analysis. The reachability and recognizability diagram for failure mode analysis (40) is found by deleting arcs (b) and (d) from (41). In Limited, arc (a) is essentially a user input ${ }^{25}$. The failure mode analysis algorithms $S$ pec and Gen decide arc (c). Thus, in sec. 6.2 , (c) corresponds to (F1). Failure mode analysis links a weak EDR strategy $\theta_{1}$ followed by a strong EDR strategy $\theta_{2}$. (a) warrants that $G$ is a fixedpoint under $\theta_{2}$. (b) ensures that failure is preserved under $\theta_{2}$ : no serendipitous goal achievement from $H$ is possible. Thus such plans are pure disambiguation strategies.

Push-Forward Algorithms. Plans found by push-forward algorithms such as Multi admit a diagram from (41) containing arcs (a), (b), and (c), but not containing (d). The arc (b) (which is shown in detail in eq. (39)) permits serendipitous goal achievement from $H$ under $\theta_{2}$. The absence of arc (d) yields strong equivalence. Again, push-forward algorithms link a weak EDR strategy followed by a strong one. They differ from failure mode analysis plans in that the arc (b) is permissible, and (a) is not a user input. The peg-in-hole plan with model error (figs. 4-66) is an example of such a plan.

2-Step Weak EDR. A plan admitting the diagram (41) with all four arcs (a)(d) demonstrates weak equivalence. It is formed by linking together two weak EDR strategies into a 2 -Step plan. We have discussed the semantics of such plans above. The key differences between 2-step weak EDR plans and push-forward or failure-mode plans are (1) the existence of arc (d), and (2) the linking of 2 weak (as opposed to a weak and a strong) EDR strategies.

In all cases, note that the linking conditions are required. Thus the linking conditions have somewhat surprisingly turned out to be the underlying characterization for multistep EDR strategies. That is, since they are necessary and sufficient conditions for

\footnotetext{
${ }^{25}$ Although we have discussed methods for inferring (a) computationally, this is really a direction for future work rather than a focus of this research.
} 
constructing multi-step EDR plans, the linking conditions may, in fact, be taken as the definition of multi-step EDR strategies.

Hence in considering LIMITED's techniques for multi-step strategy generation, we find that both failure model analysis and push-forward algorithms are essentially special cases of the Weak EDR theory. This is summarized in the table below:

\begin{tabular}{|c|c|c|c|}
\hline Method & Arcs in (41) & Strategy Type & Comments \\
\hline Failure Mode Analysis & $\mathrm{a}, \mathrm{c}$ & weak*strong & $\begin{array}{l}\text { Pure Disambiguation. } \\
\text { (a) is user input, (c) is computed. }\end{array}$ \\
\hline Push-Forwards & $a, b, c$ & weak*strong & (b) permits serendipitous goal achieverr \\
\hline Weak EDR & $a, b, c, d$ & weak*weak & 2-Step Weak EDR. \\
\hline
\end{tabular}

\subsubsection{Algebraic Considerations}

Let us pause and review the key points in this development. Weak EDR theory, strategy equivalence, and the linking conditions were introduced as a unifying framework for planning multi-step strategies.

1. The linking conditions are necessary and sufficient criteria for admitting the composition of two weak EDR strategies $\theta_{1}^{w}$ and $\theta_{2}^{w}$ into a two-step strategy which is weakly equivalent to a one-step strong EDR strategy from $\theta_{1}$. We may write this as

$$
\theta_{1}^{w} * \theta_{2}^{w} \stackrel{w}{\simeq} \theta_{1}^{s}
$$

2. The linking conditions are necessary and sufficient criteria for admitting the composition of a weak EDR strategy $\theta_{1}^{w}$ and a strong EDR strategy $\theta_{2}^{\text {s }}$ into a two-step strategy which is strongly equivalent to a one-step strong EDR strategy from $\theta_{1}$. We may write this as

$$
\theta_{1}^{\mathrm{w}} * \theta_{2}^{\mathrm{s}} \stackrel{\mathrm{s}}{\simeq} \theta_{1}^{s}
$$

3. The gear plan is a special case of (2). In particular:

4. Failure mode analysis is a special case of satisfying the linking conditions to render a two-step EDR strategy strongly equivalent to a one-step strong EDR strategy.

5. Multi-step strategies may also be planned, by repeatedly pushing forward. This was the gist of algorithm Multi in the beginning of this chapter. Multi may be viewed as 
chaining together weak EDR strategies followed by a strong EDR strategy. Multi is also essentially a special case of $(2)$, with the goal fixed-point condition relaxed. ${ }^{26}$

\section{Conclusions}

The focus of this paper lies in the synthesis of multi-step fine-motion strategies in the presence of uncertainty. We began by reviewing a basic theory of planning. This theory-called the EDR theory-had two chief components. The first is a technique for planning compliant motion strategies in the presence of model error. The second is a precise, geometrical charaterization of error detection and recovery (EDR). These led to a constructive definition of EDR plans in the presence of sensing, control, and model error. These more general strategies are applicable in assembly planning where guaranteed plans do not exist, or are difficult to find.

A number of mathematical tools were developed for the EDR theory. First, we considered compliant motion planning problems with $n$ degrees of motion freedom, and $k$ dimensions of variational geometric model uncertainty. We reduced this planning problem to the problem of computing preimages in an $(n+k)$-dimensional generalized configuration space, which encompasses both the motion and the model degrees of freedom, and encodes the control uncertainty as a kind of non-holonomic constraint.

Next, we characterized EDR strategies geometrically via the EDR region $H$. Determining whether a strategy satisfied the EDR axioms was reduced to a decision problem about forward projections and preimages in generalized configuration space.

The Weak EDR theory introduced a new mathematical tools for studying multi-step strategies-reachability and recognizability diagrams, strong and weak strategy equivalence, linking conditions, and strategy composition. A variety of techniques for planning multi-step EDR strategies were investigated and unveiled as special rases of the Weak EDR theory. In particular, we discussed the design and implementation of push-forward algorithms, and failure-mode analysis. We tested the EDR theory by implementing a planner, LIMITED, and running experiments to have LIMITED automatically synthesize EDR strategies in the domain of planar assemblies.

Much work remains to be done in developing the theory and practice of EDR. Future research can extend the EDR framework-for example, by more sophisticated dynamic models, or by considering probabilistic strategies. In addition, further analysis of the existing framework is required. Towards this end, in [Donald, 87], we explored the complexity of EDR planning. We derived bounds both for the implemented planner LIMITED, and for theoretical extensions. While in general it is known that compliant motion planning with uncertainty is intractable, we were able to demonstrate a number of special cases where there exist efficient theoretical algorithms. In particular, we showed a case where $n=2, k=1$ and containment in the backprojection could be computed in poly-

\footnotetext{
${ }^{26}$ Relaxing this restriction was discussed in the section "Beyond the Fixed Point Restriction," above.
} 
nomial time (note for $n=3, k=0$, this is false $[\mathrm{CR} \mid$ ). We also investigated the structure of the non-directional backprojection in the plane. By applying results from computational algebra, it led to a polynomial-time algorithm for computing one-step (guaranteed) strategies, and a roughly singly-exponential algorithm ${ }^{27}$ for multi-step strategies.

In addition to structural and algorithmic extensions, a number of other research directions deserve further attention:

- The weak EDR theory, while still in its infancy, has already yielded some interesting results and a fairly clean mathematical framework for studying multi-step strategies. The key idea behind the weak EDR theory is: given a collection of goals $\left\{G_{B}\right\}$ (possibly including $H$ ), we consider all unions of the subcollections to get some measure of weakest recognizability. This is perhaps the most exciting theoretical area for future work.

- When rotations and compliant motion are allowed, we do not know of exact algorithms, even in principle, for computing projection sets. For example, the computation of forward projections is not immediately decidable within the theory of real closed fields. This is because the physics of motion are essentially specified "differentially," that is, by a mapping that sends a configuration $x \in \mathcal{G}$ and a commanded motion $\theta \in S^{n}$ (where $n+1$ is the dimension of $C$ ), to a cone $B_{c}(x, \theta)$ in the tangent space: $^{28}$

$$
\begin{array}{ccc}
\mathcal{G} \times S^{n} & \rightarrow & \text { cones in } T \mathcal{G} \\
(x, \theta) & \mapsto & B_{c}(x, \theta) .
\end{array}
$$

Thus we have a differential specification of the possible motions $B_{c}(x, \theta)$ at each point $x$. The cones at each point specify a parametric family of vector fields-a field of cones to be precise. The integral curves for this family, however, may not be algebraic in general. Good approximate algorithms are needed to construct bounding algebraic envelopes about the image of this family of curves. For example, assuming that an integral curve has a power series, it is possible to construct a recurrence relation for the coefficients of the series. They can be generated deterministically to the accuracy desired. Randy Brost ${ }^{29}$ has investigated other numerical techniques for constructing integral curves corresponsing to trajectories in the forward projection. This is an interesting area for future research. In particular, it could be applied to the "full" 4-dimensional gear meshing problem where a commanded pure rotation of the gripped gear could induce translations or rotations of either gear. Such algorithms might also be applied to compute projection sets under different dynamics.

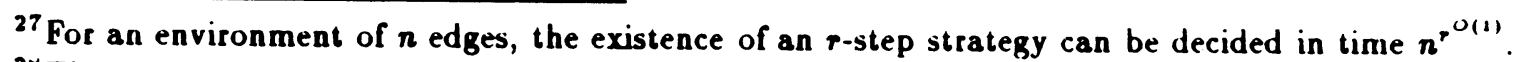
${ }^{28}$ The space of "cones in $T \mathcal{G}^{\prime \prime}$ can be formalized as an appropriate tensor bundle over $\mathcal{G}$.

${ }^{29}$ [Personal Communication].
} 


\section{Review of Previous Work}

This paper is based on |Donald, 87 . Broadly speaking, previous work falls the following ategories: Algorithmic motion planning, Compliant motion planning with uncertainty, Model Error, and Error detection and recovery.

\subsection{Algorithmic Motion Planning}

In algorithmic motion planning, (also called the piano movers' problem, or the find-path problem) the problem is to find a continuous, collision-free path for a moving object (the robot) amidst completely known polyhedral or semi-algebraic obstacles. It is assumed than once such a path is found, it can be reliably executed by a robot with perfect control and sensing. Many algorithms employ configuration space, [Lozano-Péré, Arnold, Abraham and Marsden, Udupa]. [Lozano-Pérez and Wesley] proposed the first algorithms for polygonal and polyhedral robots and obstacles without rotations. These results were later extended by [Lozano-Pérez 81, 8:3] to polyhedral robots which could translate and rotate. [Brooks 83 ] designed a find-path algorithm based on a generalized-cone representation of free-space. Brooks later extended this method for a revolute-joint robot. [Donald $84,85,87$ ] developed a motion-planning algorithm for a rigid body that could translate and rotate with six degrees of freedom amidst polyhedral obstacles (the socalled "classical" movers' problem). [Lozano-Pérez 85) reported another 6DOF algorithm for 6-link revolute manipulators. |Canny 85| developed an algebraic formulation of the configuration-space constraints, which led to a very clean collision-detection algorithm. All of these algorithms have been implemented.

There are many theoretical results on upper and lower bounds for the find-path problem, see $\mid$ Yap $\mid$ for a good survey article. These results begin with [Lozano-Pérez and Wesley], who give the first upper bounds: they give efficient algorithms for planning in $2 \mathrm{D}$ and $3 \mathrm{D}$ in the absence of rotations. [Reif 79 ] obtained the first lower bounds, demonstrating the problem to be $\mathcal{P S P} \mathcal{A C E}$-hard when the number of degrees of freedom are encoded in the input specification of the problem. [Hopcroft, Joseph, and Whitesides] and [Hopcroft, Schwartz, and Sharir] have also given intersesting lower bounds for motion planning. [Schwartz and Sharir] gave a very general theoretical algorithm for motion planning via a reduction to the theory of real closed fields. The algorithm is doublyexponential in the degrees of freedom, but polynomial in the algebraic and geometric complexity of the input. Over the next five years, there were many papers reporting more efficient special-purpose motion planning algorithms for certain specific cases; see |Yap| for a survey. To date the fastest general algorithm is due to [Canny, 87], who gives a generic motion planning algorithm which is merely singly-exponential in the degrees of freedom. For a motion planning problem of algebraic complexity $d$, geometric complexity $n$, and with $r$ degrees of freedom, Canny's algorithm runs in time $\left(d^{O\left(r^{2}\right)} n^{r} \log n\right)$ which is within a $\log$ factor of optimal. While none of these theoretical algorithms have been 
implemented, Canny's is conjectured to be efficient in practice as well.

One might ask whether exact algorithms for motion plannning can ever be utilized after uncertainty in sensing and control are introduced. The answer is a qualified "yes." In particular, the Voronoi diagram has proved to be useful for motion planning among a set of obstacles in configuration space (see [O'Dúnlaing and Yap 82; Ó'Dúnlaing, Sharir, and Yap 84; Yap 84], and the textbook of [Schwartz and Yap 86| for an introduction and review of the use of Voronoi diagrams in motion planning). The Voronoi diagram, as usually defined, is a strong deformation retract of free space so that free space can be continuously deformed onto the diagram. This means that the diagram is complete for path planning, i.e. Searching the original space for paths can be reduced to a search on the diagram. Reducing the dimension of the set to be searched usually reduces the time complexity of the search. Secondly, the diagram leads to robust paths, i.e. paths that are maximally clear of obstacles. Hence Voronoi-based motion planning algorithms are relevant to motion planning with uncertainty. [Canny and Donald] define a "Simplified Voronoi Diagram" which is still complete for motion planning, yet has lower algebraic complexity than the usual Voronoi diagram, which is a considerable advantage in motion planning problems with many degrees of freedom. Furthermore, the Simplified diagram is defined for the $6 \mathrm{D}$ configuration space of the "classical" movers' problem. For the 6 DOF "classical" polyhedral case, [Canny and Donald] show that motion planning using the Simplified diagram can be done it time $O\left(n^{\top} \log n\right)$.

Many additional robotics issues are discussed in (Paul; Brady et al.).

\subsection{Compliant Motion Planning with Uncertainty}

This section reviews previous work on planning compliant motions which are guaranteed to succeed even when the robot system is subject to sensing and control uncertainty. All of this work assumes perfect geometric models of the robot and ubstacles.

Work on compliant motion can be traced to [Inoue, Whitney, Raibert and Craig, Salisbury]. This work in force control attempted to use the geometric constraints to guide the motion. By cleverly exploiting the task geometry, placements far exceeding the accuracy of pure position control can be achieved. [Mason 83) develops spring and damper compliance models, and gives an extensive review of research in compliant motion. [Simunovic, Whitney, Ohwovoriole and Roth, Ohwovoriole, Hill and Roth/ have all considered frictional constraints, as well as jamming and wedging conditions. [Erdmann], [Burridge, Rajan and Schwartz] have considered algorithmic techniques for predicting reaction forces in the presence of friction. [Caine] has considered manual techniques for synthesizing compliant motion strategies, generalizing the methods of [Simunovic, Whitney]. [Mason, 82] has developed a way to model pushing and grasping operations in the presence of frictional contact. [Peshkin] has extended this work. [Brost] has further developed techniques for predicting pushing and sliding of manipulated objects to plan squeeze-grasp operations. In addition, Brost is currently investigating the application of 
EDR techniques to the squeeze-grasp domain.

Early work on planning in the presence of uncertainty investigated using skeleton strategies.: [Lozano-Pérez 76 ] proposed a task-level planner called LAMA which used gemmetric simulation to predict the outcomes of plans, and is one of the earliest systems to address EDR planning. [Taylor] used symbolic reasoning to restrict the values of variables in skeleton plans to guarantee success. |Brooks 82 | later extended this technique using a symbolic algebra system. [Dufay and Latombe] implemented a system which addresses learning in the domain of robot motion planning with uncertainty.

|LM'T| proposed a formal framework for automatically synthesizing fine-motion strategies in the presence of sensing and control uncertainty. Their method is called the preirnage framework. [Mason, 83] further developed the preimage termination predicates, addressing completeness and correctness of the resulting plans. [Erdmann/ continued work on the preimage framework, and demonstrated how to separate the problem into questions of reachability and recognizability. He also showed how to compute preimages using backprojections, which address reachability alone, and designed and implemented the first algorithms for computing backprojections. [Erdmann and Mason| developed a planner which could perform sensorless manipulation of polygonal objects in a tray. Their planner makes extensive use of a representation of friction in configuration space [Erdmann]. [Buckley] implemented a multi-step planner for planning compliant motions with uncertainty in 3D without rotations. He also developed a variety of new theoretical tools, including a combined spring-damper dynamic model, 3D backprojection and forward projection algorithms, and a finitization technique which makes searching the space of coinmanded motions more tractable.

|Hopcroft and Wilfong| addressed the problem of planning motions in contact, and proved important structural theorems about the connectivity of the 1-edges of configuration space obstacle manifolds. [Koutsou| has suggested a planning algorithm which plans along l-edges. Other planning systems for compliant motion have been developed by [Turk], who used backprojections, [Laugier and Theveneau], who use an expert system for geometric reasoning about compliant motion, and [Valade].

Recently, there has been some theoretical work on the complexity of robot motion planning with uncertainty. [Erdmann] showed the problem to be undecidable when the obstacles are encoded as a recursive function on the plane. [Natarajan] has shown the problem to be $\mathcal{P S P} \mathcal{A C E}$-hard in $3 \mathrm{D}$ for finite polyhedral obstacles. [Canny and Reif] have demonstrated that in $3 \mathrm{D}$ the problem of synthesizing a multi-step strategy is hard for non-deterministic exponential time; in addition, they proved that verifying a 1 -step strategy is $\mathcal{N} \mathcal{P}$-hard.

\subsection{Model Error}

There is relatively little previous work on planning in the presence of model uncertainty. [Requicha] and [Shapiro| address representational questions of how to model part tol- 
erances, and mathematical models for variational families of parts. |Buckley| considers some extensions of his planner to domains with model uncertainty. (Brooks 82) developed a symbolic algebra system which can constrain the variable values in skeleton plans, and introduce sensing and motion steps to reduce these values until the error ranges are small enough for the plan to be guaranteed. Some of the variables in these plans can represent model error-particularly, the position of objects in the workspace-and hence his planner can reason about motion planning in the presence of model uncertainty.

Work on manipulator pushing and sliding [Mason, Peshkin| and squeeze-grasping [Brost) may be viewed as addressing model error where the error parameters are the position and orientation of the manipulated part. The operation space of [Brost] is a clever example how to model actions with uncertain effects, and objects with uncertain orientation, in the same space. [Durrant-Whyte] considers how to model geonetric uncertainty probabilistically, and how to propagate such information in applications related to motion planning.

[Lumelsky| considers the following problem: suppose that a robot has a $2 \mathrm{D}$ configuration space, perfect control and sensing, the obstacles are finite in number, and each obstacle houndary is a homeomorphic image of the circle. Then a collision free-path may be found by tracing around the boundary of any obstacles encountered when moving in a straight line from the start to the goal. At each obstacle boundary encountered, there is a binary choice of which way to go, and the move may be executed with perfect accuracy. Lumelsky also demonstrates complexity bounds under these assumptions, and has considered configuration spaces such as the plane, the sphere, the cylinder, and the 2-torus. While it is not clear how this technique can extend to higher-dimensional configuration spaces, it is useful to compare Lumelsky's approach as an example of how to exploit a useful geometric primitive (wall-following). See also [Koditschek/ for extensions to this approach using potential fields. The potential-field approach to collision avoidance, as formulated by [Khatib], also can deal with uncertain obstacles, and gross motions around these obstacles can often be synthesized in real time. [Brooks 85] has described a mapmaking approach for a mobile robot in a highly unstructured environment -i.e., amidst unknown obstacles. His approach allows the robot to aquire information about the position and shape of these obstacles as the robot explores the environment. [Davis] has addressed the mobile robot navigation problem amidst partially unknown obstacles using an approximate map.

There is almost no work on planning compliant motions or assemblies in the presence of model error.

\subsection{Error Detection and Recovery}

There has been almost no formal analysis of the EDR problem. STRIPS [Fikes and Nilsson] has a run-time executive (PLANEX) which embodied one of the first systems addressing EDR. STRIPS' triangle tables may be viewed as a kind of forward projection. [Ward 
and Mc('alla; Hayes/ have presented research agendas for error diagnosis and recovery in domain-independent planning. |McDermott| has stressed the importance of EDR in plan execution and sketched an approach based on possible worlds. [Srinivas] described a robot planning system for a Mars rover which could detect certain manipulation errors and recover. [Gini and Gini] have described a view of EDR based on a predetermined list of high-level error types. The domain-independent planning literature (Chapman| is relevant to the history of EDR; for example, the planner of [Wilkins | has an error recovery module in which the executor can detect inconsistencies in the set of logical propositions representing the world state. At this point, an operator can intervene and type in new propositions to disambiguate the state and aid recovery. The robots described by [Brooks 85] have an EDR flavor - they are not required to achieve a particular goal, but merely to attenupt it until some other goal takes a higher priority.

The EDR theory in this paper has been presented in [Donald 86a,b; 87]. In [Donald, 88 ], we describe details of the geometrical characterization of EDR, and of one-step EDR planning. [Brost) is employing some of these EDR techniques in his research on planning squeeze-grasp operations.

\section{References}

C'ertuin frequently cited references have been given shorter mnemonics, eg., [LMT].

1. Abraham, R. and Marsden, J. Foundations of Mechanics, Benjamin/CumInings, London (1978).

2. Arnold, V. I. Mathematical Methods of Classical Mechanics, Springer-Verlag, New York (1978).

3. Ben-Or M., Kozen D., and Reif J., "The Complexity of Elementary Algebra and (ieometry", J. Comp. and Sys. Sciences, Vol. 32, (1986), pp. 251-264.

4. Boyse J. W., "Interference Detection Among Solids and Surfaces", Comm ACM, vol 22, No 1 (1979) pp 3-9.

5. Brady, M. et. al. (eds). Robot Motion: Planning and Control., Cambridge, Mass.: MIT Press. (1982).

6. Brooks, R. A. Symbolic Error Analysis and Robot Planning, International Journal of Robotics Research, Vol 1, no. 4, Dec., 29-68 (1982).

7. Brooks, R. A A Robust Layered Control System for a Mobile Robot, IEEE Journal of Robotics and Automation RA-2 (1): 14-23. Also MIT A.I. Lab Memo 864 (1985). 
8. Brooks, R., "Solving the Find-Path Problem by Good Representation of Free Space", IEEE Transactions on Systems, Man, and C'yberbetics, Vol. 13, 1983.

9. Brooks, R., and T Lozano-Pérez, "A Subdivision Algorithm in Configuration Space for Findpath with Rotation", Eighth International Joint Conference on Ar. tificial Intelligence, harlsruhe, Germany, August, 1983.

10. Brost, R. C. Planning Robot Grasping Motions in the Presence of Incertainty, Computer Science Department and the Robotics Institute, Carnegie-Mellon Iniversity, CMU-RI-TR-85-12 (1985).

11. Brost, R., "Automatic Grasp Planning in the Presence of Uncertainty", IEEE International Conference on Robotics and Automation, San Francisco, April, 1986.

12. Brost, R. A State/Action Space Approuch to Planning Robot Actions, Forthcoming Ph.D. Thesis, Computer Science Dept., CMU (to appear).

13. Buckley, S. J. Planning and Teaching Compliant Motion Strategies, Ph.D. Thesis. Massachusetts Institute of Technology, Department of Electrical Engineering and Computer Science, 1987. Also MIT-AI-TR-936 (1987).

14. Burridge, R., Rajan, V. T., and Schwartz, J. T. The Peg-In-Hole Problem: Statics and Dynamics of Nearly Rigid Bodies in Frictional Contact, IEEE ICRA, Raleigh, NC (1983).

15. Caine, M., "Chamferless Assembly of Rectangular Parts in Two and Three Dimensions", S.M. dissertation, MIT Department of Machanical Engineering, June 1985.

16. Cameron S., "A Study of the Clash Detection Problem in Robotics", proc. IEEE conf. on Robotics and Automation, 1985, pp 488-493.

17. Canny, J.F. A New Algebraic method for Robot Motion Planning and Real Geometr.y, FOC'S (1987).

18. [C] Canny, J. F. Collision Detection for Moving Polyhedra, PAMI-8(2) (1986).

19. Canny, J.F. The Complexity of Robot Motion Planning, Ph.D. Thesis, MIT Department of Electrical Engineering and Computer Science (1987).

20. Canny, J.F. Computing Roadmaps of Compact Serni-Algebraic Sets, Intl. Workshop on Geometric Reasoning, Oxford, England, June (1986).

21. Canny, J.F. and Donald, B. R. Simplified Voronoi Diagrams, Proc. ACM Symposium on Computational Geometry, Waterloo, June (1987). 
2'2. Canny, J.F. and Donald, B. R. Simplified Voronoi Diagrams, Discrete and Computational Geometry (to appear).

2:3. [CR] Canny, J., and J. Reif, "New Lower Bound Techniques for Robot Motion Planning Problems", FOC'S (1987).

24. Chapman, D. Planning for Conjunctive Goals, MIT Al-TR 802 (1985).

25. Chistov A. L. and Grigoryev D. Y., "Complexity of quantifier elimination in the theory of algebraically closed fields", Lect. Notes (omp. Sci. 176, Springer Verlag, (1984).

26. Collins G. E. "Quantifier Elimination for Real Closed Fields by Cylindrical Algebraic Decomposition" Lecture Notes in Computer Science, No. 33, Springer-Verlag, New York, (1975), pp. 135-183.

27. Cutkosky, M., "Grasping and Fine Manipulation for Automated Manufacturing", Ph.D. dissertation, Carnegie-Mellon University, January, 1985.

28. Davis, E. and McDermott, D. Planning and Executing Routes through Uncertain Territory, Yale (Iniversity, Dept. of Computer Science (1982).

29. [D1] Donald, B. R. Motion Planning with Six Degrees of Freedom, MIT AI-TR 791, Artificial Intelligence Lab. (1984).

30. Donald, B. R. On Motion Planning with Six Degrees of Freedom: Solving the Intersection Problems in Configuration Space, IEEE International Conference on Robotics and Automation, St. Louis, MO (1985).

31. Donald, B. R. A Search Algorithm for Motion Planning with Six Degrees of Freedom, Artificial Intelligence, 31 (3) (1987).

32. [D] Donald, B. R. Robot Motion Planning with Uncertainty in the Geometric Models of the Robot and Environment: A Formal Framework for Error Detection and Recovery, IEEE International Conference on Robotics and Automation, San Francisco, April (1986a).

33. [D] Donald, B. R. A Theory of Error Detection and Recovery for Robot Motion Planning with Uncertainty, Intl. Workshop on Geometric Reasoning, Oxford, England (1986b).

34. [D] Donald, B. R. A Geometric Approach to Error Detection and Recovery for Robot Motion Planning with Uncertainty, To appear in Artificial Intelligence (1988).

35. Donald, B. R. Error Detection and Recovery for Robot Motion Planning with Uncertainty, MIT Artificial Inteligence Laboratory, MIT-AI-TR 982 (1987). 
36. Draper Laboratories, Fourth Annual Seminar on Robotics and Advanced Assembly Systems, Cambridge, Massachusetts, November, 1983.

37. Dufay, B., and J. Latombe, "An Approach to Automatic Robot Programming Based on Inductive Learning", in Brady, M., and R. Paul, Robotics Research: The First International Symposium, MIT Press, 1984.

36. Durrant-Whyte, H. Concerning Uncertain Gitonetry in Robotics, Intl. Workshop on Geometric Reasoning, Oxford, England, June (1986).

39. [E] Erdmann, M. Using Backprojections for Fine Motion Planning with I'ncertainty, IJRR Vol. 5 no. I (1986).

40. Erdmann, M. On Motion Planning With Uncertainty, MIT AI Lab, MIT-AI-TR 810 (1984).

41. Erdmann, M., and M. Mason, "An Exploration of Sensorless Manipulation", IEEE International Conference on Robotics and Automation, San Francisco, April, 1986.

42. Faverjon, B. Obstacle Avoidance Using an Octree in the Configuration Space of a Manipulator, Proc. IEEE Intl. Conf. Robotics, Atlanta (March 1984).

43. [STRIPS] Fikes, R. and Nilsson, N. STRIPS: A New Approach to the Appli. cation of Theorem Proving to Problem Solving, Artificial Intelligence vol. 2 (1971).

44. Fortune, S., Wilfong, G., and Yap, C. 1986 (April $7-10$, San Francisco, California). Coordinated Mution of Two Robot Arms. Proceedings of the 1986 IEEE International Conference on Robotics and Automation, pp. 1216-1223.

45. Gini, M. and Gini, G. Towards Automatic Error Recovery in Robot Prograrns, IJC,AI-83 (1983).

46. Grigoryev D. Y., "Complexity of Deciding Tarski Algebra" Jour. Symbolic Computation, special issue on decision algorithms for the theory of real closed fields, to appear (1987).

47. Grossman, D., and R. Taylor, "Interactive Generation of Object Models with a Manipulator", IEEE Transactions on Systems, Man, and Cybernetics, Vol. 8, No. 9, September, 1978.

48. [Gor] Gordon, B. B. Intersections of Higher-Weight Cycles over Quaternionic Modular Surfaces and Modular Forms of Nebentypus, Bull. AMS 14 (2), pp. 293-8 (1986).

49. Hayes, P. A Representation for Robot Plans, 4th IJCAI (1976). 
50. Hopcroft, J. E., Schwartz, J. T., and Sharir, M. 1984 On the Complexity of Motion Planning for Multiple Independent Objects; PSPACE-Hardness of the "Warehouseman's Problen." International Journal of Robotics Research. 3(4): 66 88.

51. Hopcroft J., and Wilfong G., "Motion of Objects in Contact," Int. Jour. Robotics Res. vol 4, no. 4, (1986).

52. Hungerford, T. W. Algebra, Springer-Verlag, New York (ITM 73 (1974).

53. Hogan, N., "Impedance Control of Industrial Robots", Robotics and ComputerIntegrated Manufacturing, Vol. 1, No. 1, 1984.

54. Inoue, H., "Force Feedback in Precise Assembly Tasks", MIT Artificial Intelligence Laboratory, AIM-308, August, 1974.

55. Khatib, O. Real-Time Obstacle Avoidance for Manipulators and Mobile Robots, Int. Jour. Rob. Res. vol. 5, No. 1, pp. 90-99 (1986).

56. Koditschek, D., "Exact Robot Navigation by Means of Potential Functions: Some Topological Considerations", Proc. IEEE Intl. Conf. Robotics, Raleigh, March 1987.

57. Koutsou, A., "A Geometric Reasoning System for Moving an Object While Maintaining Contact with Others", ACM Symposium on Computational Geornetry, Yorktown Heights, N.Y., 1985.

58. Kozen D., and Yap C. "Algebraic Cell Decomposition in NC", Proc IEEE symp. FOCS, (1985), pp. 515-521.

59. Laugier, C., "A Program for Automatic Grasping of Objects with a Robot Arm", Eleventh Symposium of Industrial Robots, Japan Society of Biomechanisms and Japan Industrial Robot Association, 1981.

60. Lee, D. T., and Drysdale, R. L., "Generalization of Voronoi diagrams in the plane," SIAM J. Comp. (10) (1981) pp. 73-87.

61. Lieberman, L., and M. Wesley, "AUTOPASS: An Automatic Programming System for Computer Controlled Mechanical Assembly", IBM Journal of Research Development, Vol. 21, No. 4, 1977, pp. 321-333.

62. Lozano-Pérez, T., "The Design of a Mechanical Assembly System", S.M. dissertation, MIT Department of Electrical Engineering and Computer Science, also AI-TR-397, MIT Artificial Intelligence Laboratory, 1976. 
63. Lozano-Pérez, T. Automatic Planning of Manipulator Transfer Movements, IEEE Trans. on Systems, Man and Cybernetics (SMC'-11):681-698 (1981).

64. Lozano-Pérez, T. Spatial Planning: A Configuration Space Approach, IEEE Trans. on Computers $(\mathrm{C}-32): 108-120$ (1983a).

65. Lozano-Pérez, T., "Robot Programming", IEEE Proceedings, 1983b.

66. Lozano-Pérez, T., "Motion Planning For Simple Robot Manipulators", Third International Symposium on Robotics Research, Paris, October, 1985.

67. Lozano-Pérez, "A Simple Motion Planning Algorithm for General Robot Manipulators," in Proceedings of Fifth National Conference for the American Association of Artificial Intelligence, Philadelphia, 1986, pp. 626-631.

68. [LMT] Lozano-Pérez, T., Mason, M. T., and Taylor, R. H. Automatic Synthesis of Fine-Motion Strategies for Robots, Int. J. of Robotics Research, Vol 3 , no. 1 (1984).

69. Lozano-Pérez, T., and Wesley, M. A. An algorithm for planning collisionfree paths among polyhedral obstacles, Communications of the ACM (22):560-570 (1979).

70. Lumelsky, V. J. Continuous Motion Planning in Unknown Environment for a $3 D$ Cartesian Robot Arm. , Proceedings of the 1986 IEEE International Conference on Robotics and Automation, pp. 1569-1574. (April 7-10, San Francisco, Calif.) (1986).

71. Mason, M.T. Compliance and force control for computer controlled manipulators, IEEE Trans. on Systems, Man and Cybernetics (SMC-11):418-432 (1981).

72. Mason, M.T. Manipulator Grasping and Pushing Operations, MIT AI Lab, MIT AI-TR-690 (1982).

73. Mason, M. T. 1986. Mechanics and Planning of Manipulator Pushing Operations. International Journal of Robotics Research 5(3).

74. Mason, M. T. Automatic Planning of Fine Motions: Correctness and Completeness, 1984 IEEE International Conference on Robotics, Atlanta Ga. (1984).

75. Mason, M. T. 1985 (March 25-28, St. Louis, Missouri). The Mechanics of Manipulation. Proceedings of the 1985 IEEE Int. Conf. on Robotics and Automation, pp. 544-548.

76. McDermott, D. A Temporal Logic for Reasoning about Processes and Plans, Cog. Sci. 6, pp. 101-55 (1982). 
77. Natarajan, B. K. 1986 (Oct. 27-29, Toronto, Ontario). An Algorithmic Approach to the Automated Design of Parts Orienters. Proceedings of the 27th Annual IEEE Symposium on Foundations of C'omputer S'cience, pp. 132 142.

ið. Natarajan, B. K. 1986. On Moving and Orienting Objects. Ph.D. Thesis. Ithaca, N.Y.: Cornell University Department of Computer Science.

79. Neivergelt, J., and Preparata, F. P. Plane-Sweep Alyorithrns for Intersecting Geornetric Figures, C'A('M Vol. 25, no. 10 (1982).

80. O'Dúnlaing, C., Sharir, M., and Yap C., "Generalized Voronoi diagrams for moving a ladder: I Topological Analysis," NYU-Courant Institute, Robotics Lab. Tech. report No. 32 (1984)

81. Ó'Dúnlaing, C., Sharir, M., and Yap C., "Generalized Voronoi diagrams for moving a ladder: II Efficient construction of the diagram," NYU-Courant Institute, Robotics Lab. Tech. report No. 33 (1984)

82. O'Dúnlaing $\mathbf{C}$., and Yap $\mathbf{C}$., "A retraction method for planning the motion of a disc," J. Algorithmıs (6) (1985) pp. 104-111

83. Ohwovoriole, M., and B. Roth, "A Theory of Parts Mating For Assembly Automation", Proceedings of the Robot and Man Symposium 81, Warsaw, Poland, September 1981.

४4. Paul, R., Robot Manipulators, MIT Press, Cambridge, Massachusetts, 1981.

85. Peshkin, M., "Planning Robotic Manipulation Strategies for Sliding Objects", Ph.D. dissertation, Department of Physics, Carnegie-Mellon University, 1986.

86. Peshkin, M., and A. Sanderson, "Reachable Grasps on a Polygon: The Convex Rope Algorithm", IEEE Journal of Robotics and Automation, Volume 2, Number 1, March, 1986.

87. Raibert, M., and J. Craig, "Hybrid Position/Force Control of Manipulators", Journal of Dynamic Systerns, Measurement, and Control, No. 102, June, 1981, pp. 126-133.

88. Reif J., "Complexity of the Mover's Problem and Generalizations," Proc. 20th IEEE Symp. FOCS, (1979). Also in "Planning, Geometry and Complexity of Robot Motion", ed. by J. Schwartz, J. Hopcroft and M. Sharir, , Ablex publishing corp. New Jersey, (1987), Ch. 11, pp. 26i-281.

ช9. Requicha, A. A. Representation of Tolerances in Solid Modeling: Issues and Alternative Approaches, Solid Modeling by Computers: From Theory to Applications; Plenum, N. Y. (1984). 
90. Salisbury, J.K., "Active Stiffness Control of a Manipulator in Cartesian Coordinates", IEEE Conference on Decision and Control, Albuquerque, New Mexico, November, 1980.

91. Salisbury, J.K., "Kinematic and Force Analysis of Articulated Hands", Ph.D. dissertation, Stanford University, Department of Mechanical Engineering, 1982.

92. Segre, A. M., and G. DeJong, "Explanation-Based Manipulator Learning: Acquisition of Planning Ability Through Observation", IEEE International Conference on Robotics and Autornation, St. Louis, March, 1985.

93. Schwartz J., Hopcroft J., and Sharir M., "Planning, Geometry and Complexity of Robot Motion Planning", Albex Publishing Co., New Jersey, (1987).

94. Schwartz J. and Sharir M., "On the 'Piano Movers' Problem, II. General Techniques for Computing Topological Properties of Real Algebraic Manifolds," Comp. Sci. Dept., New York University report 41, (1982). Also in "Planning, Geometry and Complexity of Robot Motion", ed. by J. Schwartz, J. Hopcroft and M. Sharir, Ablex publishing corp. New Jersey, (1987), Ch. 5, pp. 154-186.

95. Schwartz J. and Yap C. K., "Advances in Robotics," Lawrence Erlbaum associates, Hillside New Jersey, (1986).

96. Simunovic, S. N., "An Information Approach to Parts Mating", Ph.D. dissertation, Department of Electrical Engineering, Massachusetts Institute of Technology, 1979.

97. Simunovic, S. N. 1975 (Sept. 22-24, Chicago, Illinois). Force Information in Assembly Processes. Proceedings 5th International Symposium on Industrial Robots. Bedford, U.K.: IFS Publications, pp. 415-431.

98. Shapiro, V. Parametric Modeling and Analysis of Tolerances, GM Research Lab. Rept. CS-460 (1985).

99. Srinivas, Sankaran Error Recovery in Robot Systems, Cal. Tech. Ph.D. Thesis, Computer Science (1977).

100. Taylor, R. H. The Synthesis of Manipulator Control Programs from Task-level Specifications, Stanford Artificial Intelligence Laboratory, AIM-282, July (1976).

101. Tarski A., "A Decision Method for Elementary Algebra and Geometry" Univ. of Calif. Press, Berkeley, (1948), second ed. 1951.

102. Turk, M., "A Fine-Motion Planning Algorithm", SPIE Conference on Intelligent Robots and Computer Vision, Cambridge, Massachusetts, September, 1985. 
103. Udupa, S., "Collision Detection and Avoidance in Computer Controlled Manipulators", Ph.D. dissertation, Department of Electrical Engineering, California Institute of 'Technology, 1977.

104. Udupa S., "Collision Detection and Avoidance in Computer Controlled Manipulators", Proc. 5th Int. Joint. Conf. on Art. Intell., Mass. Inst. Tech. (1977) pp 737-748.

105. Valade, J., "Automatic Generation of Trajectories for Assembly Tasks", Sixth European Conference on Artificial Intelligence, Pisa, Italy, September, 1984.

106. Ward, B. and McCalla, G. Error Detection and Recovery in a Dynamic Planning Environment, AAAI (1983).

107. Whitney, D., "Force Feedback Control of Manipulator Fine Motions", Journal of Dynamic Systems, Measurement, and Control, June, 1977, pp. 91-97.

108. Whitney, D., "Quasi-Static Assembly of Compliantly Supported Rigid Parts", Journal of Dynamic Systems, Measurement, and Control, Vol. 104, March 1982.

109. Whitney, D., "Historical Perspective and State of the Art in Robot Force Control", IEEE International Conference on Robotics and Automation, St. Louis, March, 1985.

110. Wilkins, D. E. Domain-Independent Planning: Representation and Plan Generation, Artificial Intelligence, Vol. 22 No. 3 (1984).

111. Yap, C., "Coordinating the motion of several discs," NYU-Courant Institute, Robotics Lab. No. 16 (1984)

112. Yap, C., "Algorithmic Motion Planning", in Advances in Robotics: Volume 1, edited by J. Schwartz and C. Yap, Lawrence Erlbaum Associates, 1986. 